\title{
Frequently asked questions about in vivo chlorophyll fluorescence: practical issues
}

\author{
Hazem M. Kalaji • Gert Schansker • Richard J. Ladle • Vasilij Goltsev • \\ Karolina Bosa · Suleyman I. Allakhverdiev $\cdot$ Marian Brestic · Filippo Bussotti • \\ Angeles Calatayud • Piotr Dąbrowski • Nabil I. Elsheery • Lorenzo Ferroni • \\ Lucia Guidi - Sander W. Hogewoning - Anjana Jajoo - Amarendra N. Misra • \\ Sergio G. Nebauer · Simonetta Pancaldi • Consuelo Penella $\cdot$ DorothyBelle Poli • \\ Martina Pollastrini - Zdzislawa B. Romanowska-Duda - Beata Rutkowska • \\ João Serôdio $\cdot$ Kancherla Suresh $\cdot$ Wiesław Szulc $\cdot$ Eduardo Tambussi · \\ Marcos Yanniccari • Marek Zivcak
}

Received: 9 February 2014/ Accepted: 2 June 2014/Published online: 15 August 2014

(C) The Author(s) 2014. This article is published with open access at Springerlink.com

\begin{abstract}
The aim of this educational review is to provide practical information on the hardware, methodology, and the hands on application of chlorophyll (Chl) $a$ fluorescence technology. We present the paper in a question and answer format like frequently asked questions. Although nearly all information on the application of Chl $a$ fluorescence can be found in the literature, it is not always easily
\end{abstract}

The authors dedicate this paper to Professor Govindjee on the Occasion of his 80th Birthday

\section{H. M. Kalaji ( $ه)$}

Department of Plant Physiology, Faculty of Agriculture and

Biology, Warsaw University of Life Sciences - SGGW,

Nowoursynowska 159, 02-776 Warsaw, Poland

e-mail: hazem@kalaji.pl

\section{G. Schansker $(\square)$}

Avenue des Amazones 2, 1226 Chêne-Bougeries, Switzerland e-mail: gert.schansker@gmail.com

\section{R. J. Ladle}

Institute of Biological and Health Sciences, Federal University of Alagoas, Praça Afrânio Jorge, s/n, Prado, Maceió, AL, Brazil e-mail: richard.ladle@ouce.ox.ac.uk

\section{Goltsev}

Department of Biophysics and Radiobiology, Faculty of Biology, St. Kliment Ohridski University of Sofia, 8 Dr. Tzankov Blvd., 1164 Sofia, Bulgaria

e-mail: goltsev@gmail.com; goltsev@biofac.uni-sofia.bg

\section{K. Bosa}

Department of Pomology, Faculty of Horticulture,

Biotechnology and Landscape Architecture, Warsaw University of Life Sciences - SGGW, Nowoursynowska 159,

02-776 Warsaw, Poland

e-mail: karolinabosa@gmail.com accessible. This paper is primarily aimed at scientists who have some experience with the application of Chl $a$ fluorescence but are still in the process of discovering what it all means and how it can be used. Topics discussed are (among other things) the kind of information that can be obtained using different fluorescence techniques, the interpretation of $\mathrm{Chl} a$ fluorescence signals, specific applications of these techniques, and practical advice on different subjects, such as on the length of dark adaptation before measurement of the $\mathrm{Chl} a$ fluorescence transient.

\section{S. I. Allakhverdiev}

Institute of Plant Physiology, Russian Academy of Sciences,

Botanicheskaya Street 35, Moscow 127276, Russia

e-mail: suleyman.allakhverdiev@gmail.com

\section{S. I. Allakhverdiev}

Institute of Basic Biological Problems, Russian Academy of Sciences, Pushchino, Moscow Region 142290, Russia

\section{Brestic $\cdot$ M. Zivcak}

Department of Plant Physiology, Slovak Agricultural University,

Tr. A. Hlinku 2, 94976 Nitra, Slovak Republic

e-mail: marian.brestic@uniag.sk

M. Zivcak

e-mail:marek.zivcak@uniag.sk

F. Bussotti - M. Pollastrini

Department of Agri-Food Production and Environmental

Science (DISPAA), University of Florence, Piazzale delle

Cascine 28, 50144 Florence, Italy

e-mail: filippo.bussotti@unifi.it

M. Pollastrini

e-mail: martina.pollastrini@unifi.it 
The paper also provides the physiological background for some of the applied procedures. It also serves as a source of reference for experienced scientists.

Keywords Chlorophyll $a$ fluorescence - Fluorescence imaging $\cdot$ Complementary techniques $\cdot$ Frequently asked questions · Plant stress monitoring · Photosynthesis

\section{Abbreviations}

$A_{\mathrm{n}}$ ATP synthase

Net $\mathrm{CO}_{2}$ assimilation rate

Car

Enzyme responsible for the synthesis of ATP from ADP and inorganic phosphate

Chl

Chlz

CP43, CP47 Carotenoid

Cyt $b_{6} / f$ D1 protein Chlorophyll

Accessory chlorophyll in the photosystem II reaction center

Core antenna proteins of PSII of 43 and $47 \mathrm{kDa}$

Cytochrome $b_{6} / f$ complex

One of the major PSII reaction center proteins, the other being D2

DBMIB

DCMU 2,5-Dibromo-3-methyl-6-isopropyl-pbenzoquinone

3-(3,4-Dichlorophenyl)-1,1-

dimethylurea

DF

Delayed fluorescence

ETC

Electron transport chain

ETR

Electron transport rate

$F_{\mathrm{O}}$

Minimum Chl $a$ fluorescence yield in the dark-adapted state

$F_{\mathrm{M}}$ Maximum Chl $a$ fluorescence yield in the dark-adapted state
$F_{\mathrm{V}}$

$F_{\mathrm{q}}^{\prime}$

Agricultural Botany Department, Faculty of Agriculture, Tanta

University, Tanta, Egypt

e-mail: nshery@yahoo.com

L. Ferroni $\cdot$ S. Pancaldi

Department of Life Sciences and Biotechnologies, University of Ferrara, Corso Ercole I d'Este 32, 44121 Ferrara, Italy

e-mail: frrlnz@unife.it
$F_{\mathrm{t}}$

$F_{\mathrm{V}} / F_{\mathrm{M}}$

$F_{\mathrm{O}} / F_{\mathrm{M}}$

$F_{\mathrm{O}}^{\prime}, F_{\mathrm{V}}^{\prime}, F_{\mathrm{M}}^{\prime}, F_{\mathrm{S}}$

$F_{\mathrm{q}}^{\prime} / F_{\mathrm{M}}^{\prime}$

$\mathrm{Fd}$

FER

FNR

$I_{1}$

IRGA

LED

LHCII

$\mathrm{NADP}^{+}$

NPQ

OJIP

S. Pancaldi

e-mail: pcs@unife.it

L. Guidi

Department of Agriculture, Food and Environment, Via del

Borghetto, 80, 56124 Pisa, Italy

e-mail: lucia.guidi@unipi.it

S. W. Hogewoning

Plant Lighting BV, Veilingweg 46, 3981 PC Bunnik, The

Netherlands

e-mail: info@plantlighting.nl

A. Jajoo

School of Life Sciences, Devi Ahilya University,

Indore 452 001, M.P, India

e-mail: anjanajajoo@hotmail.com

Fluorescence intensity at time $\mathrm{t}$ variable fluorescence, as $F_{\mathrm{M}}-F_{\mathrm{O}}$ steady state fluorescence intensity in the light-adapted state

$F_{\mathrm{M}}{ }^{\prime}-F^{\prime}$ [with $F^{\prime}=F \mathrm{~s}$ in the steady state]

Photosystem II operating efficiency

Ferredoxin

Fluorescence excitation ratio

Ferredoxin-NADP ${ }^{+}$-reductase

Fluorescence intensity at 2-3 ms

Infra red gas analyzer

Light-emitting diode

Light harvesting complex II

Nicotinamide adenine dinucleotide phosphate, oxidized form

Non-photochemical quenching, expressed as $\left(F_{\mathrm{M}} / F_{\mathrm{M}}{ }^{\prime}-1\right)$

transient Chl a fluorescence rise induced during a dark-to-strong light transition, where $\mathrm{O}$ is equivalent to $F_{\mathrm{O}}, P$ is for peak, equivalent to $F_{\mathrm{M}}$ (when measured at saturating light) and $\mathrm{J}$ and $\mathrm{I}$ are inflections between $\mathrm{O}$ and $\mathrm{P}$

\section{A. N. Misra}

Centre for Life Sciences, Central University of Jharkhand, Ratu-

Lohardaga Road, Ranchi 835205, India

e-mail: misraan@yahoo.co.uk; misra.amarendra@gmail.com 
$\mathrm{J}\left(F_{\mathrm{J}}\right), \mathrm{I}\left(F_{\mathrm{I}}\right)$,

$\mathrm{P}\left(F_{\mathrm{P}}\right)$

P680

P700

PAM

PFD

PEA

PIabs

PQ

PSI, PSII

$Q_{\mathrm{A}}$

$Q_{\text {B }}$

$q \mathrm{E}, \mathrm{qT}, \mathrm{qI}$

$\mathrm{qN}$

$\mathrm{qP}$

RLC

ROS

Rubisco

S-states S0

S1, S2, S3

and $\mathrm{S} 4$

\section{S. G. Nebauer}

Departamento de Producción vegetal, Universitat Politècnica de

València, C de Vera sn, 46022 Valencia, Spain

e-mail: sergonne@bvg.upv.es

D. Poli

Department of Biology, Roanoke College, 221 College Lane,

Salem, VA 24153, USA

e-mail: poli@roanoke.edu

\section{Z. B. Romanowska-Duda}

Department of Ecophysiology and Plant Development,

University of Lodz, Banacha 12/16, Lodz 90-237, Poland

e-mail: romano@biol.uni.lodz.pl

B. Rutkowska - W. Szulc

Agricultural Chemistry Department, Faculty of Agriculture and

Biology, Warsaw University of Life Sciences - SGGW,

Nowoursynowska 159, 02-776 Warsaw, Poland

e-mail: beata_rutkowska@sggw.pl

W. Szulc

e-mail: wieslaw_szulc@sggw.pl
$\mathrm{Sm}$

STF

TL

$\mathrm{XC}$

$\mathrm{UV}$

$\Delta V_{\text {IP }}$

$\Phi \mathrm{CO}_{2}$

$\Phi_{\text {PSII }}$

\section{Introduction}

The measurement of chlorophyll (Chl) $a$ fluorescence is one of the most widely used methods to probe photosynthesis (see Papageorgiou and Govindjee 2004 for reviews on application of Chl $a$ fluorescence to different aspects of photosynthesis; also see Govindjee (2004) for an overview of important publications on $\mathrm{Chl} a$ fluorescence). Any researcher who tries to find his or her way in the fluorescence literature will initially be overwhelmed by the number of published articles and by all the conflicting ideas. Such a researcher will also quickly discover that it is not easy to find an answer for many simple and basic questions. We plan to fill this gap in this educational review focusing mainly on plants, green algae, and diatoms.

The Chl $a$ fluorescence signal is very rich in its content; it is very sensitive to changes in photosynthesis and can be recorded with great precision. Many processes affect the fluorescence yield and/or intensity, and using a variety of light protocols (flashes, pulses, continuous light, etc.), different processes can be studied. However, most authors have used only a limited set of experimental protocols based on methods that have been developed over time.

J. Serôdio

Departamento de Biologia, CESAM - Centro de Estudos do

Ambiente e do Mar, Universidade de Aveiro, Campus de

Santiago, 3810-193 Aveiro, Portugal

e-mail: jserodio@ua.pt

K. Suresh

Directorate of Oil Palm Research, West Godavari Dt.,

Pedavegi 534 450, Andhra Pradesh, India

e-mail: sureshkancherla@ rediffmail.com

E. Tambussi - M. Yanniccari

Institute of Plant Physiology, INFIVE (Universidad Nacional de

La Plata - Consejo Nacional de Investigaciones Científicas y

Técnicas), Diagonal 113 №495, 327 La Plata, Argentina

e-mail: tambussi35@yahoo.es

M. Yanniccari

e-mail: marcosyanniccari@ conicet.gov.ar 
With the available commercial equipment, it is very easy to make a fluorescence measurement, but as the literature shows, the interpretation of such measurements is still very contentious. There is not even agreement on the processes that determine the fluorescence rise from $F_{\mathrm{O}}$ to $F_{\mathrm{M}}$, i.e., the variable fluorescence $\left(F_{\mathrm{V}}\right)$. The dominant interpretation assumes that the variable fluorescence is determined by the redox state of $Q_{\mathrm{A}}$, the first quinone acceptor of PSII, as originally proposed by Duysens and Sweers (1963) and recently defended by Stirbet and Govindjee (2012). Delosme (1967) on the other hand argued that $Q_{\mathrm{A}}$ was not enough and that there was another important process explaining part of $F_{\mathrm{V}}$. This position has recently been supported and extended by Schansker et al. (2011, 2014); see Question 21 for a broader discussion of this point.

Another attractive feature of $\mathrm{Chl} a$ fluorescence is its non-invasive character, which allows the measurement on leaves and even on canopies of trees during long periods of time. A range of instruments has been developed focusing on different aspects of photosynthesis and on different properties of $\mathrm{Chl} a$ fluorescence. An overview will be given here of the available types of instruments, and we will discuss also what kind of information can be obtained with these instruments.

It is important to understand that a fluorescence value by itself has no meaning. A well-defined reference state for the photosynthetic sample measured is needed to allow an appropriate interpretation of the data. Processes that relax following illumination will be discussed here as well as the time needed to reach the dark-adapted state, which is an important reference state.

A widely read introductory paper on the use of $\mathrm{Chl}$ $a$ fluorescence is by Maxwell and Johnson (2000), and two more recent papers treating the application of Chl $a$ fluorescence techniques are by Logan et al. (2007) and Murchie and Lawson (2013). These papers focus on the analysis of what is called the steady state: the stable photosynthetic activity after 5-10 min of illumination at a chosen light intensity. Here, our focus is broader, considering a wider range of fluorescence techniques. We make the point that interpretation of fluorescence data can be improved making use, at the same time, of different classes of fluorescence techniques, as well as by the use of complementary techniques such as gas exchange and $820 \mathrm{~nm}$ transmission/absorption measurements. We also emphasize that there are still controversies with respect to the interpretation of $\mathrm{Chl} a$ fluorescence data.

The educational review is meant to be a starting point for researchers interested in further exploiting Chl $a$ fluorescence measurements to understand photosynthetic systems. Some questions arise are trivial, e.g.,
Question 1: should the instrument be called fluorimeter or fluorometer?

Both versions are allowed, the former being British-English and the latter American-English.

Answers to other questions may make the difference between a successful and a failed experiment.

\section{Question 2. Which types of instruments are available for fluorescence measurements?}

For a rough classification of fluorescence instruments used to probe electron transfer reactions involving photosystem II (PSII) and/or photosystem I (PSI), three major classes can be distinguished (see Fig. 1 for an illustration of this classification and see Question 33 for a discussion of fast repetition rate (FRR) measurements and equipment).

[1] Instruments based on short light flashes (few $\mu$ s or less). With such instruments, information on the electron transfer reactions within PSII can be obtained: re-oxidation kinetics of $Q_{\mathrm{A}}^{-}$via forward electron transfer to $Q_{\mathrm{B}}$ or recombination with the donor side of PSII (see Fig. 2).

[2] Instruments based on a saturating pulse (few hundred ms strong light). With such instruments, information on the photosynthetic electron transport chain (ETC) can be obtained: reduction kinetics of the ETC, PSII antenna size, relative content of ETC components like PSI (see Fig. 3).

[3] Instruments designed to study the steady state (relatively stable photosynthetic activity after 5-10 min of illumination). With such instruments, light-induced regulatory mechanisms, interaction between ETC, Calvin-Benson cycle, stomatal opening, and photorespiration (the process initiated when the enzyme Rubisco reacts with $\mathrm{O}_{2}$ instead of $\mathrm{CO}_{2}$ ) are studied (see Fig. 4).

Flash fluorescence measurements

Figure 2 shows an example of a typical flash fluorescence experiment. These measurements are based on the concept of a single turnover flash (STF). An STF has to meet two requirements: (1) The intensity of a STF must be high enough to excite the antennae of all PSII reaction centers (RCs) followed by a charge separation in all PSII RCs leading to a reduction of essentially all $Q_{\mathrm{A}}$; (2) A STF must be short enough to induce only one charge separation in each PSII RC. In practice, this situation is never completely reached, and either misses or double hits are induced in a 


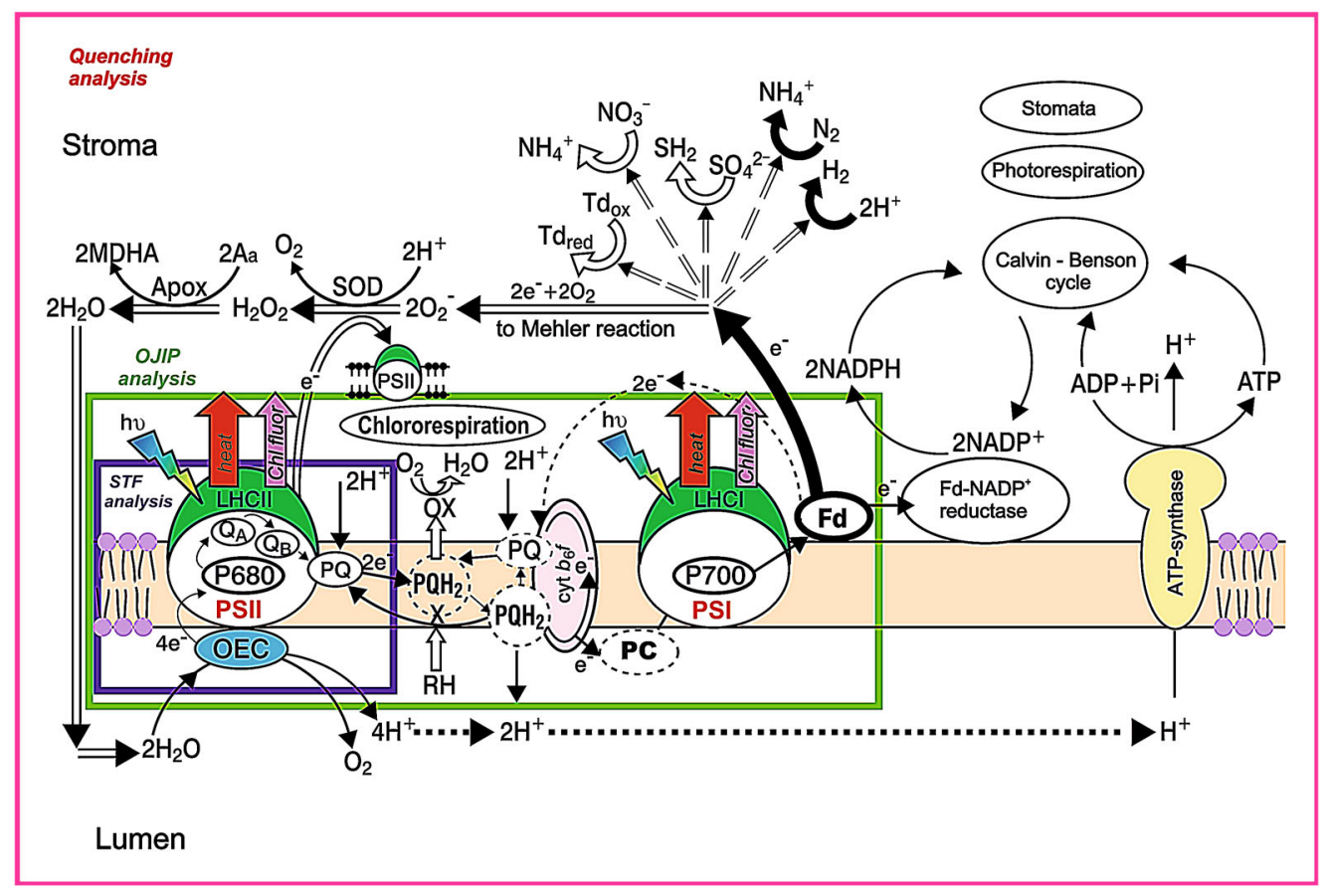

Fig. 1 The processes that can be studied analyzing the fluorescence decay following a single turnover flash, the analysis of OJIP transients, or the quenching analysis. With the analysis of the fluorescence decay kinetics (STF analysis, purple line), it is possible to obtain information on electron transport reactions inside PSII and via the occupancy state of the $Q_{\mathrm{B}}$-site on the PQ-pool redox state; OJIP transients (green line) can be used to obtain information on the

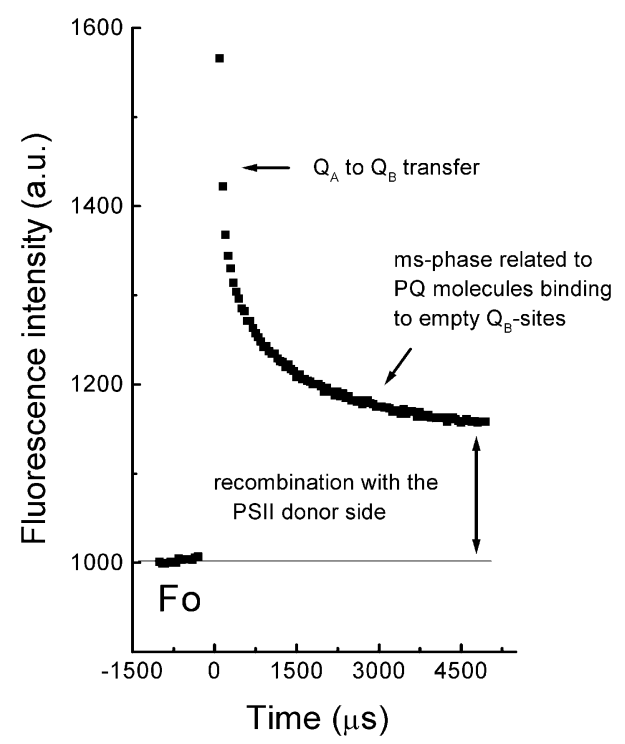

Fig. 2 Example of the fluorescence decay kinetics following a single turnover xenon flash to a suspension of PSII-enriched membranes isolated from spinach. Several pre-flashes had been given to induce a partial reduction of the PQ-pool (G. Schansker, unpublished data)

small fraction of PSII RCs (see e.g., Kok et al. 1970; Shinkarev 2005). The re-oxidation of $Q_{\mathrm{A}}^{-}$can then be followed: in active RCs, most electrons will be transferred to redox state of the photosynthetic ETC, on the stoichiometry of the components of the ETC and on the relative PSII antenna size; the quenching analysis (rosa line) gives information on dynamic processes, electron flow, under steady state conditions, is sensitive to short-term regulatory processes in the antenna (see text) and to Calvin-Benson cycle activity, changes in photorespiration and stomatal opening (modified from Kalaji and Loboda 2010)

$Q_{\mathrm{B}}$ and following a second flash to $Q_{\mathrm{B}}^{-}$(see Fig. 2). The first reaction has a half-time of 100-200 $\mu$ s, and the second reaction has a half-time of $400-600 \mu$ s (reviewed by Petrouleas and Crofts 2005). If no PQ is bound to the $Q_{\mathrm{B}}$-site, the electron on $Q_{\mathrm{A}}^{-}$has to wait, till a PQ molecule binds to the $Q_{\mathrm{B}}$-site, and this process can take a few ms (Crofts and Wraight 1983). In the case of inactive PSII centers, forward electron transfer cannot take place, and re-oxidation of $Q_{A}-$ occurs via a recombination reaction with the donor side of PSII (Lavergne 1982a; Chylla et al. 1987; Lavergne and Leci 1993; Schansker and Strasser 2005). These instruments can also be used to study the S-states (oxidation states S0, S1, S2, S3 and S4) of the oxygen evolving complex of PSII. A series of STFs induces period-4 oscillations in the $F_{\mathrm{O}}$-level as a function of the S-states (see Delosme 1972; Delrieu 1998; Ioannidis et al. 2000 for examples of such measurements).

To probe the oxidation of reduced $Q_{\mathrm{A}}$ following a saturating flash, there are two possible approaches:

(1) The easiest method makes use of low-intensity modulated light, which excites only a small fraction of the PSII RCs per unit of time. Figure 2 shows an example of such a measurement. For control samples, in which re-oxidation of $Q_{\mathrm{A}}^{-}$via forward electron 

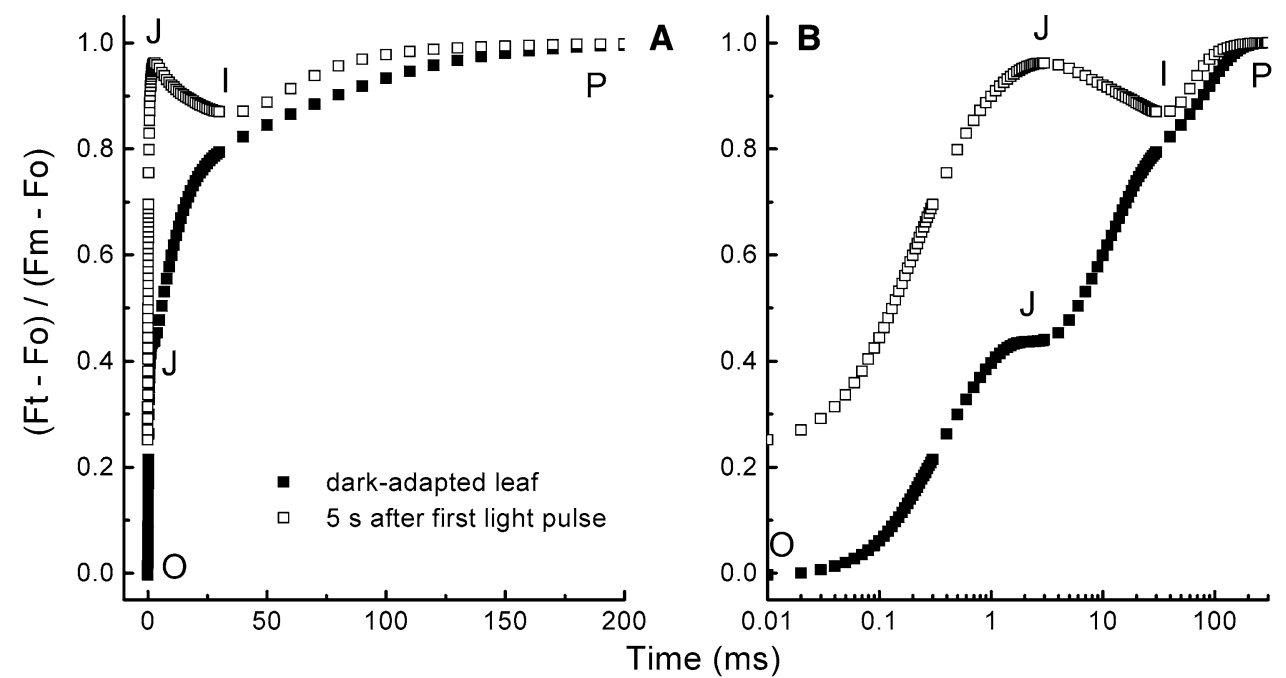

Fig. 3 OJIP transients (double normalized between $O$ and $P$ ) measured on a bean leaf (Phaseolus vulgaris) shown on a linear timescale (a) and a logarithmic timescale (b). A measurement on dark adapted (closed symbols) which has an oxidized PQ-pool and a low $\mathrm{J}$-step and a measurement made $5 \mathrm{~s}$ later (open symbols) where $Q_{\mathrm{A}}$

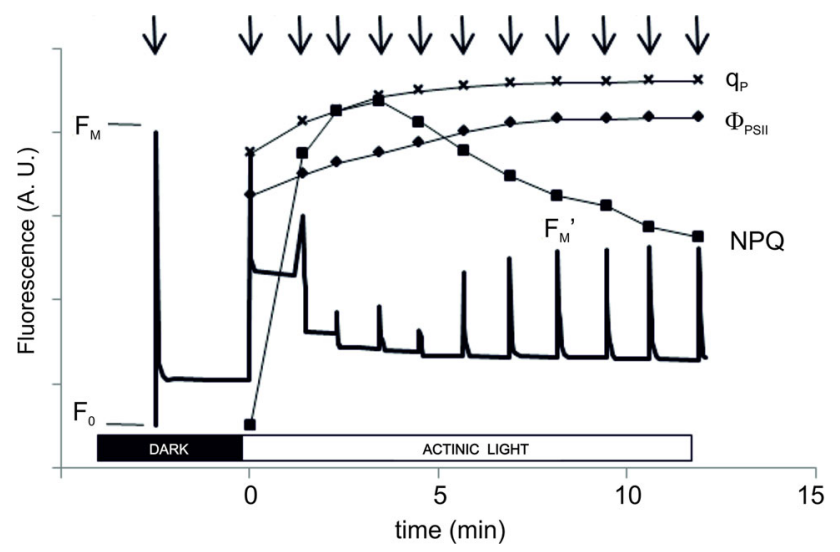

Fig. 4 Slow Chlorophyll $a$ fluorescence kinetics (in arbitrary units) using a PAM-2100 fluorometer. The dark-adapted leaf is illuminated with weak modulated measuring light to give the zero fluorescence level $F_{0}$. Application of a saturation pulse (SP) allows measurement of the maximum fluorescence level in the dark $F_{\mathrm{M}}$. Photosynthesis is then activated by an actinic light source (in this case $250 \mu \mathrm{mol}$ photons $\mathrm{m}^{-2} \mathrm{~s}^{-1}$ ). SPs during the light phase were triggered spaced $1 \mathrm{~min}$ apart (indicated by arrows) to determine the maximum fluorescence intensity in the light $\left(F_{\mathrm{M}}{ }^{\prime}\right)$, and for each SP, $\mathrm{q}_{\mathrm{P}}, \Phi_{\mathrm{PSII}}$, and NPQ parameters were calculated, and these are indicated in the figure (Penella et al. unpublished data)

transport can occur, this approach works well. However, when the sample is inhibited, e.g., by an electron transfer inhibitor such as DCMU (3-(3,4-dichlorophenyl)-1,1-dimethylurea), which displaces $Q_{\mathrm{B}}$ from its binding site (Velthuys 1981; Lavergne 1982b), the low-intensity modulated light leads to the accumulation of a considerable population of $Q_{\mathrm{A}}^{-}$complicating had become re-oxidized in part of the PSII RCs due to recombination (O level considerably below P), the PQ-pool is still almost completely reduced ( $\mathrm{J}$ level near P), and the acceptor side of PSI is almost completely re-oxidized (I level close to that of the dark-adapted state) (G. Schansker, unpublished data)

the analysis of the experiment, because re-oxidation of $Q_{\mathrm{A}}^{-}$by recombination with the donor side is much slower than forward electron transport to $Q_{\mathrm{B}}$.

(2) The second method uses a combination of a STF followed by a probe flash that probes the redox state of $Q_{\mathrm{A}}$ at the time of the probe flash (this is called a pumpprobe experiment) (Mauzerall 1972; Robinson and Crofts 1983). The intensity of the probe flash is much lower than that of the STF. In this case, the experiment is repeated many times and each time at a variable time $t$ after the STF, a probe flash is given to probe the redox state of $Q_{\mathrm{A}}$. In this way, the re-oxidation kinetics are constructed point by point. The actinic light problem, described above for DCMU inhibited samples, does not exist in this case. On the other hand, identical samples do not exist, and therefore, the biological variability between samples will lead to experimental noise and the need for repetitions to obtain smooth kinetics. To make different phases in the re-oxidation kinetics visible, the use of a logarithmic time scale has been introduced (see e.g., Cser and Vass 2007). Commercial equipment to make this type of measurements is the superhead fluorometers (Photon Systems Instruments, Brno, Czech Republic), which can also be used to measure OJIP transients and saturating pulse protocols (see below).

Complementary techniques for flash fluorescence measurements are thermoluminescence (TL) (reviewed by Vass and Govindjee 1996; Misra et al. 2001a, b; Ducruet and Vass 2009) and delayed fluorescence (DF) (recently 
reviewed by Goltsev et al. 2009) measurements that provide specific information on recombination reactions within PSII RCs.

Flash fluorescence measurements are frequently used to study PSII mutants (e.g., Etienne et al. 1990; Nixon et al. 1991; Cser and Vass 2007) and can also be used in the case of treatments that affect the function of PSII [e.g., stresses like heat stress (Yamasaki et al. 2002)] or to probe the PQ redox state (Dannehl et al. 1996).

\section{Saturating pulse or OJIP measurements}

Upon a dark-to-light transition, the fluorescence intensity of a leaf or other photosynthetic samples increases from a low value $\left(F_{\mathrm{O}}\right.$ or $\left.\mathrm{O}\right)$ via two intermediate steps $\left(F_{\mathrm{J}}\right.$ or $J$ and $F_{\mathrm{I}}$ or $\left.I\right)$ in $200-300 \mathrm{~ms}$ to a maximum value $\left(F_{\mathrm{M}}\right.$ or $\left.\mathrm{P}\right)$ during the application of a saturating pulse of light (see Fig. 3a, b; Strasser and Govindjee 1991; Strasser et al. 1995). The different fluorescence rise phases (OJ, JI and IP) can be related to different steps of the reduction of the ETC: OJ parallels the reduction of the acceptor side of PSII $\left(Q_{\mathrm{A}}+Q_{\mathrm{B}}\right)$; JI parallels the reduction of the PQ-pool and IP parallels the reduction of the electron transport acceptors in and around PSI (Schansker et al. 2005). This means that OJIP transients give information on the state of the ETC. Although complex simulations of OJIP transients use a kinetic model based on the gradual reduction of the ETC (see e.g., Lazár 2003; Zhu et al. 2005), it has been shown that the transients can also be approximated assuming that the transients consist of three kinetic components (Boisvert et al. 2006; Vredenberg 2008; Joly and Carpentier 2009) indicating that the rate limitations (exchange of PQ at the $Q_{\mathrm{B}}$-site of PSII and re-oxidation of $\mathrm{PQH}_{2}$ by cyt $\mathrm{b}_{6} / \mathrm{f}$ ) quite effectively separate the three rise phases kinetically. The kinetics of the OJIP transient are, e.g., sensitive to the PQ redox state (Tóth et al. 2007a) and PSI content (Oukarroum et al. 2009; Ceppi et al. 2012). During the isolation of thylakoid membranes, the properties of the ETC are modified, and this is reflected by changes in the fluorescence kinetics. Attempts have been made (see e.g., Bukhov et al. 2003) to make the fluorescence induction kinetics of thylakoid membranes look more like those of leaves.

Using a pulse-probe approach, a first pulse reduces the ETC and a second probe pulse given at time $t$ after the first pulse probes the redox state of the ETC. The analysis of the regeneration kinetics of the OJIP transient gives information on the rate of re-oxidation of $Q_{\mathrm{A}}^{-}$by recombination with the donor side of PSII, the re-oxidation of the PQ-pool due to plastoquinol oxidase activity (see Question 17), and the rate of re-oxidation of the acceptor side of PSI in darkness (Schansker et al. 2005).

Complementary techniques for OJIP measurements are $820 \mathrm{~nm}$ absorbance/transmission measurements that probe the redox state of PSI (plastocyanin, P700 and ferredoxin) and DF measurements that give information on the occurrence of recombination reactions in PSII as a function of the redox state of the ETC. The interpretation of these measurements can also be improved by determining the chl $a / b$ ratio and the chl content of the leaves/cells. OJIP measurements have been used widely to study the effects of stress (see Questions 19, 24, 26-28).

Steady state measurements

The steady state refers to the relatively stable photosynthetic activity that is obtained when leaves or other photosynthetic samples are illuminated at a chosen light intensity during approximately 5-10 min (or more). The Chl $a$ fluorescence intensity in the steady state is affected both by the redox state of the ETC (and $Q_{\mathrm{A}}$ in particular) and by changes in the fluorescence yield, i.e., a change in the probability that absorbed light is emitted as Chl $a$ fluorescence. These yield changes not only can be due to the formation of the transthylakoid $\Delta \mathrm{pH}$ (Krause et al. 1983) and xanthophyll cycle (XC) related changes (Bilger and Björkman 1991), antenna size changes-for example, due to state transitions, which are especially obvious for algae such as Chlamydomonas reinhardtii (see e.g., Iwai et al. 2008)—or photoinhibition (see e.g., Björkman and Demmig 1987; Van Wijk and Krause 1991; Tyystjärvi and Aro 1996) but are also due to the activation of ferredoxin NADP $^{+-}$reductase (FNR) on the acceptor side of PSI (Schansker et al. 2006, 2008). In the 1980s, an analysis was developed, called the quenching analysis (see Question 15 for a more detailed discussion of the quenching analysis) that can distinguish between redox changes (photochemical quenching) and fluorescence yield changes. A fluorescence yield change occurs when the rate constant for either fluorescence or heat emission changes. If this leads to a smaller $F_{\mathrm{M}}$ value (and in many cases smaller $F_{\mathrm{O}}$ value), this is called non-photochemical quenching. Figure 4 gives an example of such a protocol. Just as in the case of the flash fluorescence measurements (see above), the fluorescence intensity is probed using low-intensity modulated light. The steady state is induced using continuous actinic light of a chosen intensity, and in addition every 100 or $200 \mathrm{~s}$ (this can be variable time interval), a saturating pulse (comparable to an OJIP transient) is given to reduce the ETC and all $Q_{\mathrm{A}}$. On turning off the actinic light, relaxation of the induced non-photochemical quenching can be followed using saturating light pulses to probe changes in the $F_{\mathrm{M}}$ level. In general, three relaxation phases are observed (Demmig and Winter 1988; Horton and Hague 1988): the qE which relaxes within 100-200 s as a consequence of the dissipation of the transmembrane $\Delta \mathrm{pH}$, the $\mathrm{qT}$, whose relaxation is complete within $15 \mathrm{~min}$ and the $\mathrm{qI}$ which 
covers all processes that need more than 15 min to recover. As will be discussed later in detail (see Question 15) the qT and $\mathrm{qI}$ are less well defined. It is worth mentioning here that by measuring $\mathrm{Chl} a$ fluorescence induced by the saturating pulses with a higher time resolution (i.e., measuring OJIPs), it is possible to obtain more information on the character of the qT and qI phases (Schansker et al. 2006). The relaxation of the different non-photochemical quenching phases can be treated as the sum of three exponentials (see e.g., Walters and Horton 1991; Roháček 2010; and Question 15).

Obtaining the 'maximum' $F_{\mathrm{M}}{ }^{\prime}$ value is not a trivial issue. Markgraf and Berry (1990) and Earl and Ennahli (2004) observed that in the steady state, high light intensities are needed to induce the maximum $F_{\mathrm{M}}^{\prime}$ value. Earl and Ennahli (2004) observed that more than $7,500 \mu$ mol photons $\mathrm{m}^{-2} \mathrm{~s}^{-1}$ (the maximum intensity of their light source) were needed to reach the maximum $F_{\mathrm{M}}{ }^{\prime}$ value of their maize leaves and that at higher actinic light intensities, more light was needed to saturate $F_{\mathrm{M}}{ }^{\prime}$. Schansker et al. (2006) observed the same actinic light intensity dependence measuring both fluorescence and $820 \mathrm{~nm}$ transmission and suggested that the ferredoxin/thioredoxin system that is thought to continuously adjust the activity of several Calvin-Benson cycle enzymes (see Question 6), is responsible for the actinic light intensity dependence. Earl and Ennahli (2004) proposed an extrapolation method based on the measurement of $F_{\mathrm{M}}{ }^{\prime}$ at two light intensities to obtain the true $F_{\mathrm{M}}{ }^{\prime}$ value. Loriaux et al. (2013) studied the same light intensity dependence of $F_{\mathrm{M}}{ }^{\prime}$ and proposed the use of a single multiphase flash lasting approximately $1 \mathrm{~s}$ to determine the maximum $F_{\mathrm{M}}{ }^{\prime}$ value. This flash consists of two high light intensity phases separated by a short interval at a lower light intensity during which the fluorescence intensity decreases. The second high light intensity phase of this protocol has a higher light intensity than the first phase (see also Harbinson 2013 for a commentary on this paper).

Complementary techniques for this type of fluorescence measurement are gas exchange measurements (to probe Calvin-Benson cycle activity, stomatal opening, $\mathrm{CO}_{2}$ conductance) and $820 \mathrm{~nm}$ absorbance/transmission measurements.

\section{$77 \mathrm{~K}$ fluorescence spectra}

Low temperature (77 K) fluorescence measurements represent another technique to obtain information on the photosystems. At room temperature, variable fluorescence is emitted nearly exclusively by PSII. Byrdin et al. (2000) detected only a small difference in the quenching efficiencies of $\mathrm{P} 700$ and $\mathrm{P} 700^{+}$at room temperature. This is supported by the observation that inhibiting PSII by DCMU (Tóth et al. 2005a) or cyt $b_{6} / f$ by DBMIB (Schansker et al. 2005) does not affect $F_{\mathrm{M}}$ despite a big difference in the redox state of
P700 in the absence and presence of inhibitors. However, variable fluorescence emitted by PSI can be induced on lowering the temperature to $77 \mathrm{~K}$. Although measurements of light-induced fluorescence changes can be made at $77 \mathrm{~K}$, in most cases, the fluorescence emission spectrum $(600-800 \mathrm{~nm})$ is measured. This type of measurement is used to obtain information on the PSII and PSI antennae. A common application of $77 \mathrm{~K}$ measurements is the detection of the occurrence of state transitions (e.g., Bellafiore et al. 2005; Papageorgiou and Govindjee 2011; Drop et al. 2014), where changes in the relative amplitudes of the PSII and PSI bands are indicators for this process. Figure 5 gives an example of a measured $77 \mathrm{~K}$ spectrum. Emission bands at 685 and $695 \mathrm{~nm}$ are related to the antenna of PSII, and peaks around $730 \mathrm{~nm}$ are related to the antenna of PSI (Govindjee 1995; Špunda et al. 1997; Srivastava et al. 1999).

Complementary techniques are ultrafast femto- or picosecond absorbance or fluorescence measurements that give information on energy transfer within the antenna (e.g., Gilmore et al. 1998; Richter et al. 1999) but which are beyond the scope of this educational review.

Fast fluorescence techniques (ns, ps, fs time range)

As noted in the previous paragraph, fast fluorescence (and absorption) techniques, which probe energy transfer between chlorophylls or between carotenoids and chlorophylls in the photosynthetic antennae and the charge

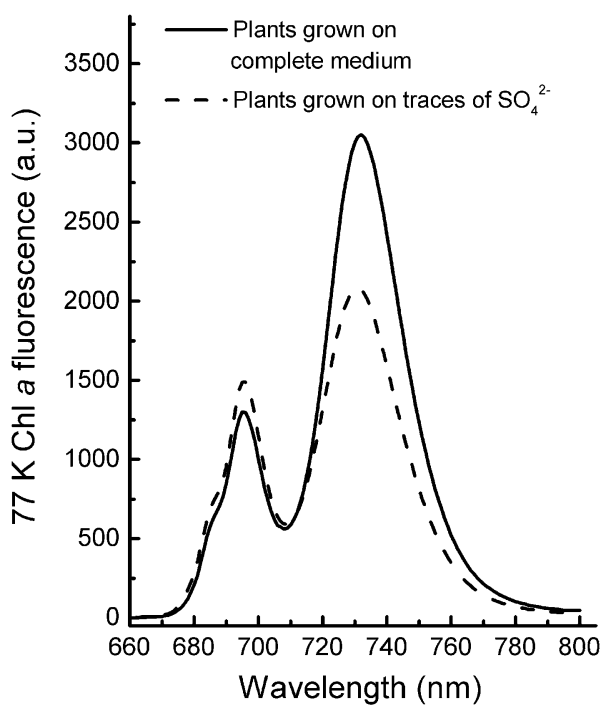

Fig. $577 \mathrm{~K}$ fluorescence emission spectra of leaves of plants grown hydroponically on a complete medium (black line) and on medium containing only traces of sulfate (green line). Sulfate deficiency led to extensive chlorosis and in addition to a rather specific loss of PSI. This reduced the long wavelength bands around $730 \mathrm{~nm}$ and increased the 685 and 695 bands due to a decreased re-absorption by PSI reaction centers of $\mathrm{Chl} a$ fluorescence emitted by PSII (Schansker and Ceppi, unpublished data) 
separation processes in the RCs of PSII and PSI will not be discussed in this paper. See e.g., Holzwarth $(1996,2008)$ and Berera et al. (2009) for introductory reviews on the application of these methods.

\section{Question 3. What is the effect of wavelengths at which the fluorescence is measured on the character of the fluorescence signal?}

Most commercial instruments measure $\mathrm{Chl} a$ fluorescence at wavelengths longer than $700 \mathrm{~nm}$. At room temperature, at wavelengths longer than $700 \mathrm{~nm}$, PSI becomes an important source of fluorescence emission. As shown by Genty et al. (1990) and Pfündel (1998) in C3 plants, about $30 \%$ of the $F_{\mathrm{O}}$ emission is due to PSI fluorescence, and in C4 plants, this percentage is even higher (Pfündel 1998). This causes, e.g., a systematic underestimation of the $F_{\mathrm{V}}{ }^{\prime} /$ $F_{\mathrm{M}}{ }^{\prime}$ value, which is used as a measure of the maximum quantum yield of PSII. Detecting Chl $a$ fluorescence emission at wavelengths below $700 \mathrm{~nm}$ can considerably reduce this problem. However, in measuring equipment such as photosynthetic efficiency analyser (PEA) and HandyPEA instruments (Hansatech Instruments Ltd, UK) which use red LEDs with an emission peak around $650 \mathrm{~nm}$, this would have led to an overlap between the actinic wavelengths and the detecting wavelengths. With the introduction of (strong) LEDs emitting at shorter wavelengths, e.g., in the blue (see e.g., Nedbal et al. 1999), it is now technically possible to avoid this overlap and to detect fluorescence below $700 \mathrm{~nm}$. Interference of PSI fluorescence at wavelengths longer than $700 \mathrm{~nm}$ should be taken into account especially when measuring fluorescence parameters in the light-adapted state. Non-photochemical quenching induced in the light quenches the variable fluorescence $\left(F_{\mathrm{M}}-F_{\mathrm{O}}\right)$ to a larger extent than $F_{\mathrm{O}}$ fluorescence. This makes the underestimation of the true $F_{\mathrm{V}}{ }^{\prime} / F_{\mathrm{M}}{ }^{\prime}$ value light intensity dependent as well, since a higher light intensity induces more non-photochemical quenching.

\section{Question 4. Which part of the leaf is probed and analyzed by a fluorescence measurement?}

The leaf is optically complex. In a dorsiventral leaf, the palisade parenchyma cells have been shown to act as light guides, keeping the light more or less focused (Vogelmann and Martin 1993; Vogelmann et al. 1996). The lobed cells of the spongy mesophyll and the spaces that surround these cells, on the other hand, disperse the light (Vogelmann and Martin 1993). At the same time, there is a strong light gradient within the leaf (Vogelmann 1989, 1993). This means that the light intensity decreases rapidly as light penetrates into the leaf. As a consequence, illuminating and probing Chl $a$ fluorescence emission on the adaxial surface of the leaf, chloroplasts located deep in the leaf will be excited by a much lower photon flux density than those located close to the adaxial side of the leaf (Terashima and Saeki 1985; Fukshansky and Martinez von Remisowsky 1992). At the same time, the spectral distribution of the light changes as well: as light penetrates the mesophyll, the relative contribution of green and far-red (FR) light progressively increases, because the absorption of these wavelengths by the leaf is less efficient (Sun et al. 1998; Rappaport et al. 2007). The chloroplasts located deeper in the leaf, i.e., those of the spongy tissue, acclimate to these lower, FR-enriched light intensities by increasing the antenna size of PSII, reducing the number of RCs, and decreasing the PSI/PSII ratio (Terashima et al. 1986; Evans 1999; Fey et al. 2005; Pantaleoni et al. 2009). Since the emitted fluorescence is a linear function of the light intensity (Vogelmann and Evans 2002; cf. Schansker et al. 2006), chloroplasts located deeper in the leaf will contribute to a lesser extent to the detected fluorescence signal. In practice, fluorescence measurements will probe mainly chloroplasts in the palisade parenchyma cells (Vogelmann and Evans 2002). The assumption that not all chloroplasts are assayed is supported by the observation that a fivefold decrease in the chlorophyll content of the leaf does not affect the detected $F_{\mathrm{O}}$ and $F_{\mathrm{M}}$ values (Dinç et al. 2012). In fact, since the total amount of fluorescence emitted by the leaf does not change, it suggests that the light beam probes deeper in the leaf as more chlorophyll is lost. The optical properties of the leaf also mean that measurements made on the abaxial (bottom) side of the leaf have characteristics that differ considerably from those made on the adaxial (top) side of the leaf (Schreiber et al. 1977). Oxygen and $\mathrm{CO}_{2}$ assimilation measurements on the other hand assay the whole leaf, and this may lead to deviations when comparing, for example, measurements of the oxygen evolving activity with fluorescence measurements (Björkman and Demmig 1987; Tyystjärvi and Aro 1996).

Given the gradient of photosynthetic properties that exists within the leaf (Terashima et al. 1986; Evans 1999), the photosynthetic response of a leaf depends on the wavelength composition of the exciting light. Deeper penetrating green light probes more low light acclimated chloroplasts located in the lower cell layers than blue light that is strongly absorbed by the leaf and mainly probes chloroplasts close to the adaxial side of the leaf.

\section{Question 5. How to dark-adapt leaves?}

For the interpretation of $\mathrm{Chl} a$ fluorescence measurements, it is important that the state of the photosynthetic apparatus 
at the beginning of the measurement is well defined. The dark-adapted state of the leaf is a well-defined state of the photosynthetic apparatus and, therefore, for most experiments, photosynthetic samples are first dark adapted.

There are four main methods to achieve dark adaptation in leaves:

1. In the case of an intact plant, a leaf can be put into a leaf clip shielding it from ambient light. However, if the ambient light intensity is high, and the leaf is not entirely flat, there is a chance that some stray light reaches the shielded area.

2. Detached leaves can be kept for a while between wet filter paper in darkness and subsequently measured in the laboratory. Detachment of leaves has consequences for the physiological state of the leaf: it causes, for example, a closure of the stomata (Raschke 1970). See Potvin (1985) and Weng et al. (2011) for a comparison of the properties of attached and detached leaves and Kato et al. (2002) for a discussion of the differences between leaves and leaf disks.

3. Under laboratory conditions, measurements can be made in the dark or in a dimly lit room under conditions that induce very little photosynthetic activity. Traditionally, low-intensity green light has been used as a kind of safe light (see Sun et al. 1998 for a discussion of this point) although we note that leaves can still absorb and use most of the green light for photosynthesis (cf. Sun et al. 1998; Vogelmann and Evans 2002; Rappaport et al. 2007).

4. Loss of time for dark adaptation can be avoided when the measurements are made directly in the field at night (no need for leaf clips). In this case, the leaves are allowed to dark adapt for many hours, and the results of such measurements differ from measurements on leaves following a relatively short dark-adaptation period during the day.

\section{Question 6. What is a "good" dark-adaptation time?}

Dark adaptation of samples that will be used for Chl $a$ fluorescence measurements, is often associated with the re-oxidation of $Q_{\mathrm{A}}^{-}$. However, dark adaptation is a considerably more complicated process, and there are more factors that can affect a subsequent fluorescence measurement.

In dark-adapted leaves, several enzymes are inactivated to prevent wasteful reactions. Examples of such enzymes include Rubisco (e.g., Streusand and Portis 1987); four other thioredoxin-dependent enzymes: D-fructose1,6-bisphosphatase, phosphoribulokinase, and sedoheptulose-1, 7-bisphosphatase (Buchanan 1984; Scheibe 1990) and ATP synthase (Stumpp et al. 1999); and FNR (Carillo et al. 1981; Satoh 1981). These enzymes are active in the light, and during a light-to-dark transition, they gradually become inactive again. The half-time of inactivation of Rubisco under in vivo conditions is 2-4 min (Stitt et al. 1987; Laisk and Oja 1998). Inactivation of ATP synthase and the three other Calvin-Benson cycle enzymes is under control of the thioredoxin system (Scheibe 1990), and their inactivation depends on the re-oxidation of stromal components such as ferredoxin and NADPH. FNR inactivation varies depending on the species: pea leaves need $\sim 15$ min for full inactivation (Schansker et al. 2006), whereas in a Pinus species, an hour is needed (Schansker et al. 2008). Once inactivated, all of these enzymes must first be activated again before steady state photosynthesis is induced, and this affects the fluorescence induction kinetics (see Papageorgiou et al. 2007; Papageorgiou and Govindjee 2011 for an in-depth discussion of the fluorescence kinetics beyond $\mathrm{P}$ or $F_{\mathrm{M}}$ in a variety of photosynthetic organisms). In addition, active FNR (i.e., an activated acceptor side of PSI) has an effect on the IP phase of the OJIP transients and on the amplitude of the $F_{\mathrm{M}}$ that can be reached by a strong pulse of light (Schansker et al. 2008). In most fluorescence studies, many are not interested in the processes mentioned above, and in that case, it is best to make the dark-adaptation time long enough to allow at least FNR to become inactive again (a marker for this is a regeneration of the fluorescence IP phase and in addition a regeneration of $820 \mathrm{~nm}$ re-reduction phase paralleling the IP phase, see Schansker et al. 2006, 2008).

As mentioned in Question 2 Sect. 3, several regulatory and stress-related processes that affect the fluorescence yield (quench $F_{\mathrm{M}}$ ) are induced in the light. Following a light-to-dark transition, i.e., on turning off the light, these processes are reversed. State transitions (the transfer of a part of the antenna system among PSII and PSI) and XC related processes may take a considerable amount of time to reverse (Fork and Satoh 1986; Ruban and Horton 1999) and the recovery of a plant from photoinhibition takes hours (Havaux 1989; Long et al. 1994).

An answer to the question as to what a good darkadaptation time is, depends on the information we want to obtain. If the aim is the study of the regulatory and photoinhibition-related processes, a dark-adaptation time of 15 min that allows FNR (at least in plants like pea) to become inactive again would be sufficient. If someone is interested in long term adaptation responses of a leaf or other photosynthetic organism to a treatment, much longer dark-adaption times that allow also the regulatory processes and processes like photoinhibition to recover may be considered (see also the next question). 
Question 7. How to obtain the best reference $F_{O}$ and $F_{M}$ values for the quenching analysis?

In field experiments, predawn measurements are often used to obtain reference $F_{\mathrm{O}}$ and $F_{\mathrm{M}}$ values for measurements made during the day (Logan et al. 1999; Maxwell and Johnson 2000; Demmig-Adams et al. 2006). Under these conditions, NPQ is assumed to be completely relaxed and therefore zero, and the photoinhibition induced during the previous day is expected to have been reversed (Flexas et al. 1998; Logan et al. 1999; Demmig-Adams et al. 2006). However, in some cases, chronic photoinhibition occurs, which can be easily detected by lowered predawn $F_{\mathrm{V}} / F_{\mathrm{M}}$ values (Osmond and Grace 1995; for a review see Demmig-Adams et al. 2012). We note that the absence of light during recovery experiments may prevent a full repair of photoinhibitory (Greer et al. 1986) and heat stress damage (Tóth et al. 2005b). Light is needed for the synthesis of ATP, which is needed for the synthesis of the D1 protein (Kuroda et al. 1992). Edhofer et al. (1998) have reported that light is needed for translation elongation of the D1 protein; these are processes that are part of the PSII repair cycle following damage to PSII (recently reviewed by Nixon et al. 2010). Low-intensity actinic light generates the ATP needed for the PSII repair cycle, and at the same time, it does not induce additional photoinhibition and is thereby more effective than a complete dark recovery (see e.g., Elsheery et al. 2007).

\section{Question 8. What can go wrong during a fluorescence measurement on leaves? Technical issues}

To dark-adapt leaves in the field, leaf clips have been developed. They cover the area of the leaf to be measured. The measuring head of, for example, a HandyPEA can be connected to a leaf clip, after which the clip can be opened, and the measurement made. Since such measurements are normally evaluated afterward, it should be kept in mind that unopened or partially opened leaf clips are a common reason for transients showing no or little fluorescence rise. A smooth leaf can also lead to problems, since the clip may shift while attaching the measuring head, and in that case, a non-dark-adapted part of the leaf will be measured. If the leaf is not flat, some stray light may enter the leaf clip via the spaces left between the leaf clip and the leaf surface. Especially on a bright day, this may prevent a full dark adaptation of the covered leaf area. The same problems can occur with pulse amplitude modulated (PAM) type instruments developed for field applications, which use leaf clips to allow dark adaptation.

When working with a PAM instrument, the measuring light intensity must be chosen in such a way that the $F_{\mathrm{M}}$ stays within the measuring window. If the measured signal is too strong, then the highest values will be cut off. For example, as a rule of thumb the fluorescence intensity induced by the measuring light (associated with $F_{\mathrm{O}}$ ) should be approximately $10 \%$ of the total scale. In any case, absolute values and their limits depend on the manufacturer, and its instructions should be carefully read before starting any measurements. Further, the distance between the leaf and the fiber optics has to be adjusted; it is usually set between 1 and $1.5 \mathrm{~cm}$. Background fluorescence signals from the environment must be suppressed by zeroing the signal in the absence of a leaf sample.

Using direct fluorescence equipment like the HandyPEA, there is also a risk that the emitted fluorescence intensity causes an overload of the detector. It is therefore important to check if, at a given gain and excitation light intensity, the measured fluorescence kinetics remain below the maximum measurable fluorescence intensity. If the emitted fluorescence intensity is too strong, then the top part of the transient will be cut off, and in that case, the gain has to be reduced.

\section{Question 9. Why was it so difficult to determine the $\boldsymbol{F}_{\mathrm{O}}$ before 1985 ?}

It may be hard to imagine nowadays, but the determination of a correct $\mathrm{F}_{\mathrm{O}}$ value was a major problem for researchers using Chl $a$ fluorescence up to the mid-1980s (see Kalaji et al. 2012a, b for a historical overview of instrument development). The shutters used at the time had a full opening time of anywhere between $0.8 \mathrm{~ms}$ (e.g., Neubauer and Schreiber 1987) and $2 \mathrm{~ms}$. At high light intensities, the J-step is reached after $\sim 0.8-2 \mathrm{~ms}$ of illumination. To minimize the effect of the shutter opening time, in many studies, low-intensity light was used to slow down the fluorescence induction kinetics. In the 1980s, two fundamentally different solutions for the shutter problem were introduced in the form of modulated systems (Schreiber et al. 1986) and PEA-type instruments (Strasser and Govindjee 1991). These two measuring concepts are explained and compared in Questions 10 and 11.

\section{Question 10. What is the principle of modulated fluorescence measurements?}

Modulated systems, pulse amplitude modulated fluorometers, (PAM) use a trick to separate the effect of the actinic light that drives photosynthesis and the low-intensity measuring light that is used to probe the state of the photosynthetic system on the measured fluorescence intensity (see also Question 2 Sect. 3). A so-called lock in amplifier 
only registers the fluorescence changes induced by the modulated measuring light and ignores the fluorescence changes induced by the continuous actinic light. This way the low-intensity measuring light can be used to measure both the $F_{\mathrm{O}}$ (induced by the measuring light itself) and $F_{\mathrm{M}}$ (induced by a strong light pulse) values (Schreiber et al. 1986). The effective light intensity of modulated light depends on the pulse frequency. In the case of a modern PAM instrument, the modulated measuring light consists of 1-3 $\mu$ s flashes of red or white light, and flash frequencies between 100 and $20,000 \mathrm{~Hz}$ can be chosen. At the lowest frequency, the effective photosynthetic photon flux density is $<0.2 \mu \mathrm{mol}$ photons $\mathrm{m}^{-2} \mathrm{~s}^{-1}$; an intensity that is 200 times higher when the highest frequency is chosen. The choice of a low frequency gives not only a very small actinic effect (= measuring-light-induced $F_{\mathrm{V}}$ ) but also a relatively poor signal-to-noise ratio. A high frequency not only is considerably more actinic but gives also a much better signal-to-noise ratio. The actinic effect of the measuring light becomes especially visible (and problematic) if PSII electron transfer inhibitors such as DCMU are being used (see Question 2 Sect. 1). Compared to PEA-type instruments an advantage of the modulated fluorimeters is that the measured fluorescence yield is independent of the intensity of both the actinic light and light of the saturating pulse (Schreiber et al. 1986). In the case of PEA-type instruments, the measured fluorescence intensity is a linear function of the actinic light intensity used, and as a consequence, the measured fluorescence intensities must be normalized first (e.g., divided by the light intensity) before measurements made at different light intensities can be compared (see e.g., Schansker et al. 2006).

\section{Question 11. What is the principle of direct fluorescence measurements?}

In the so-called direct fluorescence instruments-i.e., instruments in which the actinic light that drives photosynthesis is also used as measuring light-the $F_{\mathrm{O}}$ problem is solved by using strong light emitting diodes (LEDs): light sources that can be switched on/off very quickly (Strasser and Govindjee 1991). In modern equipment, a stable light intensity emitted by the LEDs is reached in less than $10 \mu \mathrm{s}$. Initially, only red $(650 \mathrm{~nm})$ LEDs were available for this type of measurement but now colors like other orange (discussed by Oxborough 2004), green (Rappaport et al. 2007), and blue (Nedbal et al. 1999) or a mix of LEDs of different colors (Schreiber 1998) are also available. In the original PEA instrument, the response time of the LEDs was still in the order of the 40-50 $\mu$ s (e.g., Strasser et al. 1995 ) necessitating the use of extrapolation to estimate the $F_{\mathrm{O}}$ value; in the current instruments, a response time of
$10-20 \mu \mathrm{s}$ is good enough for an accurate determination of the $F_{\mathrm{O}}$ value for light intensities below $\sim 10,000 \mu \mathrm{mol}$ photons $\mathrm{m}^{-2} \mathrm{~s}^{-1}$ (cf. Schansker et al. 2006). The absence of a measuring light source means that between pulses, there is true darkness. As a consequence, the $F_{\mathrm{O}}$ can be determined more accurately than in the case of a modulated system (see Schansker and Strasser 2005 for a discussion on the effects of very low light intensities on the $F_{\mathrm{O}}$ value). The absence of measuring light is particularly advantageous when the samples to be analyzed have been inhibited with electron transfer inhibitor such as DCMU. Another important difference between PEA instruments and modulated PAM instruments is the data sampling strategy. In PEA instruments, the data sampling is non-linear. In HandyPEA instruments, during the first $300 \mu$ s of illumination one measuring point is collected every $10 \mu \mathrm{s}$; between $300 \mu$ s and $3 \mathrm{~ms}$ one point per $100 \mu$ s, between 3 and $30 \mathrm{~ms}$ one point per ms, and between 30 and $300 \mathrm{~ms}$ one point per $10 \mathrm{~ms}$. In this way, an OJIP transient measured at a high time resolution is defined by approximately 120 measuring points. In the case of a PAM instrument, a measurement with the same initial time resolution would yield at least 20,000 measuring points (for $200 \mathrm{~ms}$ ). This makes the HandyPEA files much easier to handle when analyzing them using spreadsheet programs like Microsoft Excel.

\section{Question 12. Why use a logarithmic timescale to visualize fluorescence transient measurements?}

As described above, PEA instruments allow a shutter-less measurement of OJIP transients. However, PEA instruments make use of a second innovation and that is the use of a logarithmic timescale to visualize the measurements of the OJIP fluorescence rise (Strasser and Govindjee 1991). Bannister and Rice (1968) had already used this idea more than 20 years earlier, but at that time, it was not picked up by others. The logarithmic timescale was later exploited by researchers measuring fluorescence relaxation following a STF, as well (see Question 2 Sect. 1; e.g., Cser and Vass 2007). The logarithmic time scale distorts the time dependence somewhat but, at the same time, allows the visualization of considerably more kinetic features than is possible on a linear time scale. This additional kinetic detail makes it much easier to detect changes in the fluorescence kinetics. Fluorescence measurements shown on a linear timescale are always dominated by the slower changes (see Fig. 3a). A logarithmic timescale turns exponential rise phases into sigmoidal rise phases, and we must keep in mind that the sigmoidicity of the fluorescence rise cannot be derived on the basis of fluorescence transients visualized on a logarithmic timescale. 


\section{Question 13. Direct or modulated fluorescence?}

It is possible to measure OJIP transients using a modulated system (Schreiber 1986; Neubauer and Schreiber 1987; Schreiber and Neubauer 1987), and at the same time, it is possible to make a quenching analysis (see Questions 2.3 and 15) using a PEA-type instrument (Schansker et al. 2006). However, modulated instruments are much better suited for a quenching analysis, and PEA-type instruments are the instruments of choice for a study of the OJIP kinetics. Thus, we recommend that both must be used to get a complete picture.

\section{Question 14. What kind of additional information can be obtained using fluorescence imaging?}

All the instruments, discussed thus far, integrate the signal of the measured area. Fluorescence imaging permits the study of spatial heterogeneities in the fluorescence emission intensity within cells, leaves, or whole plants; heterogeneities caused by a range of internal plant factors (Gorbe and Calatayud 2012). It can also be used to average and analyze the fluorescence signal from much larger leaf areas than classical methods would allow, and at the same time, it allows the simultaneous measurement/screening of many samples/mutants in, for example, a microwell plate or of colonies grown on a Petri dish (see e.g., Niyogi et al. 1997; Serôdio et al. 2012) or all the leaves of an rosette of Arabidopsis. There are several commercial imaging instruments on the market. It is a technique whose development has kept pace with improvements in LED technology. For reliable imaging measurements, it is critical that the whole sample area be illuminated homogeneously. Several introductory texts and reviews have been published on fluorescence imaging (e.g., Buschmann et al. 2001; Oxborough 2004; Lenk et al. 2007; Scholes and Rolfe 2009). Since it was not possible to image $F_{O}{ }^{\prime}$ with the imaging systems available in the late 1990s, Oxborough and Baker (1997) derived an equation to estimate it:

$F_{\mathrm{O}}{ }^{\prime}=\frac{F_{\mathrm{O}}}{\frac{F_{\mathrm{V}}}{F_{\mathrm{M}}}+\frac{F_{\mathrm{O}}}{F_{\mathrm{M}^{\prime}}}}$.

This equation allows the calculation of the parameters $\mathrm{qP}\left[=\left(F_{\mathrm{M}}{ }^{\prime}-F_{\mathrm{S}}\right) /\left(F_{\mathrm{M}}{ }^{\prime}-F_{\mathrm{O}}{ }^{\prime}\right)\right]$ and $F_{\mathrm{V}}{ }^{\prime} / F_{\mathrm{M}}{ }^{\prime}$. The challenge using fluorescence imaging is to process all the data collected in a scientifically meaningful way. Meyer and Genty (1998) analyzed their data making frequency distributions of parameters of interest; we recommend that this method is considered for future experiments.

Imaging can be used, e.g., to assess the dynamics and heterogeneous behavior of stomatal opening/closure over a leaf, a phenomenon also called stomatal patchiness. A palette of false colors is used to cover the range of fluorescence intensities (normalized between 0 and 1), assigning a color to each pixel of the image (Gorbe and Calatayud 2012). Based on the image, different areas of the leaf can be chosen, the associated fluorescence data averaged, fluorescence parameters can be calculated, and subsequently, the photosynthetic properties of the chosen area can be studied.

Using fluorescence imaging, it is easy to detect photosynthetic heterogeneities in a leaf (Meyer and Genty 1998) and to follow how any stress affects the leaf in spatial terms. In a popular early experiment, the imaging technique was used to show the gradual infiltration of PSII inhibiting herbicides in the leaf (e.g., Daley et al. 1989; Lichtenthaler et al. 1997; Chaerle et al. 2003) or the effect of reactive oxygen species (ROS)-inducing herbicides (e.g., Hideg and Schreiber 2007). Spatial heterogeneities that have been studied using fluorescence imaging include heterogeneities occurring during the following processes: induction of photosynthesis (Genty and Meyer 1995; Daley et al. 1989), the onset of senescence (Wingler et al. 2004), chilling (Hogewoning and Harbinson 2007), the response to drought (Woo et al. 2008), nutrient stress (Landi et al. 2013), ozone stress (Gielen et al. 2006; Guidi et al. 2007), wounding (Quilliam et al. 2006), and during infection with viruses (Balachandran et al. 1994) or fungi (Guidi et al. 2007). Several studies, using imaging to study Chl $a$ fluorescence parameters under various conditions (high/low ambient $\mathrm{CO}_{2}$ concentration, high/low light intensity, etc.), have yielded information on the relationship between leaf structure and organization on the one hand and the response to stress conditions on the other (Baker 2008; Roháček et al. 2008; Guidi and Degl'Innocenti 2011; Gorbe and Calatayud 2012).

Serôdio et al. (2013) have introduced, a new application of fluorescence-imaging systems, which allows the rapid generation of light-response curves (see Question 18) simultaneously illuminating replicates of samples using spatially separated beams of actinic light of different intensities.

\section{Question 15. What kind of information can be obtained using the quenching analysis (see Question 2)?}

In leaves exposed to a certain irradiance, the fluorescence intensity is affected by changes both in the redox state of the ETC (particularly the redox state of $Q_{\mathrm{A}}$ ) and in the fluorescence yield due to light-induced changes in the properties of the PSII antenna. A method called the quenching analysis was developed to separate these two types of process. In most cases, the quenching analysis is used to describe the steady state, i.e., the stable 
photosynthetic activity, which is usually reached after approximately 5-10 min of illumination at a chosen actinic light intensity.

A protocol was developed (Schreiber et al. 1986; Fig. 4) based among others on the work of Bradbury and Baker (1981) in which the measurements are initiated by switching on the measuring light to determine the $F_{\mathrm{O}}$ value of a dark-adapted sample. A saturating light pulse is then applied to determine the $F_{\mathrm{M}}$. The measurement is continued switching on an actinic light source to induce photosynthesis, until the fluorescence emission stabilizes at a level called $F_{\mathrm{S}}$. The $F_{\mathrm{M}}{ }^{\prime}$ is then determined by applying another strong pulse of light followed some time later (e.g., $10 \mathrm{~s}$ ) by turning off the actinic light. Turning off, the actinic light will cause a quick, partial, re-oxidation of the photosynthetic ETC. Within the first $100 \mathrm{~ms}$ of darkness, the PQ-pool will be largely re-oxidized by forward electron transport toward $\mathrm{PC}^{+}$and $\mathrm{P} \mathrm{C0}^{+}$, and a value close to $F_{\mathrm{O}}{ }^{\prime}$ can be measured. The $F_{\mathrm{O}}{ }^{\prime}$ level subsequently increases again due to non-photochemical reduction of the PQ-pool by NADPH and possibly $F \mathrm{~d}_{\text {red }}$ (Mano et al. 1995; Gotoh et al. 2010; Guidi and Degl'Innocenti 2012). This so-called " $F_{\mathrm{O}}$ ' rise" can be almost completely suppressed by a short pulse of FR light (e.g., of $1 \mathrm{~s}$ duration) following the turning off of the actinic light. The increase of the fluorescence intensity from $F_{\mathrm{S}}$ to $F_{\mathrm{M}}{ }^{\prime}$ is related to a change in the redox state of the ETC, whereas the difference between $F_{\mathrm{M}}{ }^{\prime}$ and the dark-adapted $F_{\mathrm{M}}$ is then a measure of the fluorescence yield change, which in the case of $\mathrm{qE}$ is associated with increased heat dissipation. In quenching analysis terminology, this approach splits the fluorescence changes into a photochemical quenching (redox related) and a non-photochemical quenching (fluorescence yield related) part. On turning off the actinic light, the relaxation of the non-photochemical quenching, i.e., the increase of $F_{\mathrm{M}}{ }^{\prime}$ to $F_{\mathrm{M}}$, can be followed and several contributing processes can be resolved (Walters and Horton 1991; Roháček 2010). Schreiber et al. (1986) introduced the parameter $\mathrm{qN}=1-F_{\mathrm{V}}{ }^{\prime} / F_{\mathrm{V}}$ to quantify changes in the non-photochemical quenching. The parameter $\mathrm{qN}$ can range between 0 and 1 , and for its calculation, the $F_{\mathrm{O}}{ }^{\prime}$ value is needed. In 1990, Bilger and Björkman (1990) introduced the parameter NPQ $=F_{\mathrm{M}} / F_{\mathrm{M}}{ }^{\prime}-1$ which has as advantages over the parameter $\mathrm{qN}$ that its range is not restricted (see Question 21 ), and in addition, it is not necessary to know the $F_{\mathrm{O}}{ }^{\prime}$ value. However, Holzwarth et al. (2013) evaluating the parameter NPQ, concluded that in this treatment of the fluorescence data, the relationship between the quenching parameter and the underlying processes becomes distorted, especially when the time dependence of NPQ is considered.

For the analysis of the relaxation kinetics of the parameter $\mathrm{qN}$ semi-logarithmic plots of $\log (\mathrm{q} N)$ versus time are made. This linearizes the slowest component. Using linear regression, the decay half-time and amplitude of this component can be determined. This component (an exponential function) can then be subtracted from the original data, and a new semi-logarithmic plot can be made of the remaining $\mathrm{q} N$. The procedure can then be repeated (e.g., Walters and Horton 1991; for a discussion of the theoretical basis of the resolution method, see Roháček 2010).

The least controversial of these kinetic processes is the process relaxing during the first 100-200 s of darkness, with a relaxation half-time of $\sim 30 \mathrm{~s}$. In quenching analysis terms, this is called the $\mathrm{qE}$ or high-energy quenching; it depends on a low lumen $\mathrm{pH}$ and is affected by the XC (reviewed by Horton et al. 1996; Müller et al. 2001; Gilmore 2004; Krause and Jahns 2004; Ballottari et al. 2012). However, the exact mechanism of the induction of the $\mathrm{qE}$ and the exact components involved in this process are still a hotly debated issue (e.g., Caffari et al. 2011; Johnson et al. 2011; Miloslavina et al. 2011). A set of mutants has been generated playing an important role in the study of the $\mathrm{q} E$, in which different components and processes related to qE have been modified (Niyogi et al. 1998). The second process, the qT, with a half-time of 5-10 min has been assigned to state II to state I transitions (transfer of LHCII units from PSI to PSII) based on the observation that it was already induced at low light intensities (Demmig and Winter 1988) and on its possible sensitivity to the phosphatase inhibitor NaF (Horton and Hague 1988). Schansker et al. (2006) studying the kinetics of the saturating pulses showed that the main fluorescence change occurring in this time interval in pea leaves is the regeneration of the IP phase suggesting that the qT reflects the inactivation of the acceptor side of PSI (the inactivation of FNR). Other processes that have been associated with the qT are some slowly relaxing component(s) of qE (Lokstein et al. 1993; Joliot and Finazzi 2010) and light-dependent movements of chloroplasts (Cazzaniga et al. 2013). In practice, there are several arguments making it doubtful that the qT is a reliable measure for state transitions. The slowest relaxation phase, the qI, which may last several hours can consist of several processes: photoinhibition of PSII and XC related changes (reviewed by Krause and Jahns 2004) and possibly also state II to state I transitions (Schansker et al. 2006) if a change in the JI amplitude is related to state transitions as suggested by Schreiber et al. (1995) for cyanobacteria. It should be noted that the rate with which these processes reverse in darkness is not necessarily the same in all photosynthetic organisms. For example, the regeneration of the IP phase parallels the qT phase in pea leaves (Schansker et al. 2006), and it is complete within $15 \mathrm{~min}$, whereas the same process in needles of Pinus halepensis takes $1 \mathrm{~h}$ (Schansker et al. 2008). 


\section{Question 16. Why is far-red light used to determine the $F_{O}$ and $F_{O}{ }^{\prime}$ values?}

For leaves, it is reasonable to assume that under most conditions, nearly all PSII RCs are in the open state $\left(Q_{\mathrm{A}}\right.$ oxidized) following dark adaptation. However, the assumption is not true for heat-stressed leaves (Ducruet 1999; Tóth et al. 2007b) and leaves that show a high rate of chlororespiration. Chlororespiration refers to the non-photochemical reduction of the plastoquinone pool by reducing equivalents derived from $\mathrm{Fd}_{\text {red }}$ or NADPH in the stroma (Bennoun 2002). Feild et al. (1998) showed a high chlororespiratory activity in light acclimated sunflower leaves following a light-to-dark transition leading to considerably higher $F_{\mathrm{O}}{ }^{\prime}$ values. This $F_{\mathrm{O}}{ }^{\prime}$ increase is due to a population of reduced $Q_{\mathrm{A}}$ associated with a more reduced PQ pool. There is redox interaction between the PQ-pool and $Q_{\mathrm{A}}$ leading to a redox-equilibrium (Diner 1977); for pea leaves, it was shown that a completely reduced PQ-pool (induced by anaerobiosis) is in equilibrium with reduced $Q_{\mathrm{A}}$ in $20 \%$ of the PSII RCs (Tóth et al. 2007a).

To assure maximum oxidation of the PQ pool, the leaf can be pre-illuminated with FR light. For this purpose, FR light in the 720-735 nm range is normally used. FR light preferentially excites PSI and thereby causes an oxidation of the PQ pool. We note that FR light can induce charge separations in PSII (Pettai et al. 2005; Schansker and Strasser 2005). Pettai et al. (2005) demonstrated that FR light at $740 \mathrm{~nm}$ still induces a low level of oxygen evolution even though the activity is three times less than that induced by FR light at $720 \mathrm{~nm}$. In practice, FR light induces about $2.5 \%$ of $F_{\mathrm{V}}$ associated with $Q_{\mathrm{B}}^{-}$in $50 \%$ of the RCs (Schansker and Strasser 2005). However, this observation is only of importance for direct fluorescence measurements, since the effects induced by FR light are also induced by the measuring beam of a modulated fluorescence instrument.

A short FR pulse $(\sim 1 \mathrm{~s}$, at $\sim 720-735 \mathrm{~nm})$ given to a light-adapted leaf has two main effects: (i) it re-oxidizes the PQ-pool within $100 \mathrm{~ms}$ and (ii) it suppresses the transient $F_{\mathrm{O}}{ }^{\prime}$ increase, which is normally observed following a light-to-dark transition (Mano et al. 1995; Gotoh et al. 2010; Guidi and Degl'Innocenti 2012). It is related to nonphotochemical reduction of the PQ-pool by NADPH or $\mathrm{Fd}_{\text {red }}$; this process is mediated by an enzyme complex called NADPH dehydrogenase (NDH) (Burrows et al. 1998). The induction of the $\mathrm{qE}$ component of non-photochemical quenching leads to a quenching of the $F_{\mathrm{M}}$ level and in many plant species to a quenching of the $F_{\mathrm{O}}{ }^{\prime}$ level as well (Bilger and Schreiber 1986; Bilger and Björkman 1991; Noctor et al. 1991). This qE quenching relaxes quickly in darkness. To determine the associated $F_{\mathrm{O}}{ }^{\prime}$ quenching accurately, the $F_{\mathrm{O}}{ }^{\prime}$ level must be determined immediately after turning off the actinic light. The nonphotochemical reduction of the PQ-pool affects the $F_{\mathrm{O}}{ }^{\prime}$ level as well, and this may complicate an accurate determination of the extent of $F_{\mathrm{O}}{ }^{\prime}$ quenching. Since the nonphotochemical reduction of the PQ-pool is a rather slow process peaking approx. $40 \mathrm{~s}$ after turning off the light (Burrows et al. 1998), and the maximum re-oxidation of the PQ-pool following lights off takes less than $100 \mathrm{~ms}$ (Ceppi 2010), the $F_{\mathrm{O}}^{\prime}$ level can be determined quite accurately before the transient non-photochemical reduction of the PQ-pool sets in. However, using $\sim 1 \mathrm{~s}$ of FR is the most straightforward approach to obtain an oxidized PQ pool.

\section{Question 17. How can the NPQ index be calculated when NPQ is formed in the dark?}

As noted in Question 16, a process called chlororespiration has been identified in higher plants (Bennoun 1982, 2002; Rumeau et al. 2007). Cyanobacteria, which are thought to be the ancestors of the chloroplast, lack mitochondria; instead they have a respiratory chain that shares the PQpool with the photosynthetic ETC (Vermaas 2001; Schmetterer and Pils 2004; Hart et al. 2005). It allows the creation of a pH gradient over the thylakoid membrane in the dark, and this gradient is utilized to synthesize ATP. In the dark, the respiratory activity in cyanobacteria is considerably higher than in higher plants. In fact, chlororespiration in higher plants is seen as a rudiment of the original respiratory chain. Also in green algae, the respiratory chain is still quite active (see Beardall et al. 2003 for a discussion of this topic). Another group of organisms that have been shown to have a high chlororespiratory activity are some microalgae, including diatoms (e.g., Caron et al. 1987). As a consequence, there is no complete relaxation of $\mathrm{qE}$ in the dark. XC activity in dark grown diatoms occurs as a result of the acidification of the thylakoid lumen due to this chlororespiratory activity (Jakob et al. 1999).

One effect of this high chlororespiratory activity in diatoms is that the $F_{\mathrm{M}}$ level of dark-adapted diatoms is lower than the $F_{\mathrm{M}}{ }^{\prime}$ observed under low actinic light (Cruz et al. 2010). This means that it is not possible to apply the commonly used NPQ equation:

$\mathrm{NPQ}=\frac{F_{\mathrm{M}}}{F_{\mathrm{M}^{\prime}}}-1$,

since the calculated value would be negative $\left[F_{\mathrm{M}}<F_{\mathrm{M}}{ }^{\prime}\right]$. A practical solution for this problem is the determination of the light-response curve (see Question 18) and to replace $F_{\mathrm{M}}$ by the maximum $F_{\mathrm{M}}{ }^{\prime}$ level measured $\left(F_{\mathrm{M}^{\prime} \text { max }}\right.$; Serôdio et al. 2006) in $\mathrm{Eq}(1)$ :

So, 
$\mathrm{NPQ}=\frac{F_{\mathrm{Mmax}}^{\prime}}{F_{\mathrm{M}^{\prime}}}-1$.

In this way, NPQ values will always be positive and approach a minimum value close to zero under conditions closely corresponding to a state with a very small transthylakoid proton gradient.

\section{Question 18. Can the time that is needed for a complete quenching analysis be shortened?}

To characterize the properties of parameters such as $\mathrm{qP}$, $\Phi_{\mathrm{PSII}}\left[=\left(F_{\mathrm{M}}{ }^{\prime}-F_{\mathrm{S}}{ }^{\prime}\right) / F_{\mathrm{M}}{ }^{\prime}\right]$ and NPQ , it is common practice to determine the light intensity dependence of these parameters (see e.g., Bilger and Björkman 1991; Gray et al. 1996; Verhoeven et al. 1997). The classical approach is to illuminate the leaf at each light intensity, until steady state is reached (see Questions 2.3 and 10). This process can be quite time-consuming, especially if the fluorescence quenching analysis is performed for field experiments.

To reduce the time needed for this type of measurement, a faster procedure was developed and called rapid light curves (RLCs) (White and Critchley 1999; Ralph and Gademann 2005). RLCs can be used to study the physiological flexibility of the photochemistry in response to rapid changes in irradiation (Guarini and Moritz 2009). Such changes occur frequently in natural environments. An RLC is a plot of the electron transport rate (ETR: $\Phi_{\text {PSII }} \times$ PFD $\times 0.5 \times$ leaf absorptivity coefficient) as a function of the actinic light intensity, which is applied for fixed short-time periods (e.g., $10 \mathrm{~s}$ or $1 \mathrm{~min}$ ). Here, PFD stands for photon flux density, and here, it is assumed that the PSI:PSII ratio is 1:1. However, this is only a rough approximation and the real ratio will differ between samples (see Question 26). For this type of analysis, two criteria are important: (1) the samples must be dark adapted, and (2) photosynthesis must be induced [activation of the Calvin-Benson cycle enzymes that become inactive during incubation in darkness (see Question 6)] before the measurement sequence is started (White and Critchley 1999). Dark adaptation of the samples allows the determination of the reference $F_{\mathrm{O}}$ and $F_{\mathrm{M}}$ values needed for the calculation of qN and/or NPQ. If light-adapted samples are used for the experiments, for which reference $F_{\mathrm{O}}$ and $F_{\mathrm{M}}$ values are missing, then the effective quantum yield $\left(\Phi_{\mathrm{PSII}}\right)$ and ETR can still be calculated, but not the non-photochemical quenching parameters, nor $\mathrm{qP}$. In other words, the best protocol consists of a dark acclimation of the sample, a weak modulated beam and a saturating pulse to determine the reference $F_{\mathrm{O}}$ and $F_{\mathrm{M}}$, respectively, and then a preillumination with a moderate light intensity (approx. $50 \%$ of the ambient light intensity applied for several minutes is appropriate for this purpose) after which the RLC protocol is applied (see Lichtenthaler et al. 2005).

Examples of RLCs (Fig. 6a) illustrate the importance of the duration of light intervals. In addition to differences in the values determined for individual light intensities, there is also a difference in the shape of the curves (Fig. 6b). Preillumination at moderate light intensities ensures faster

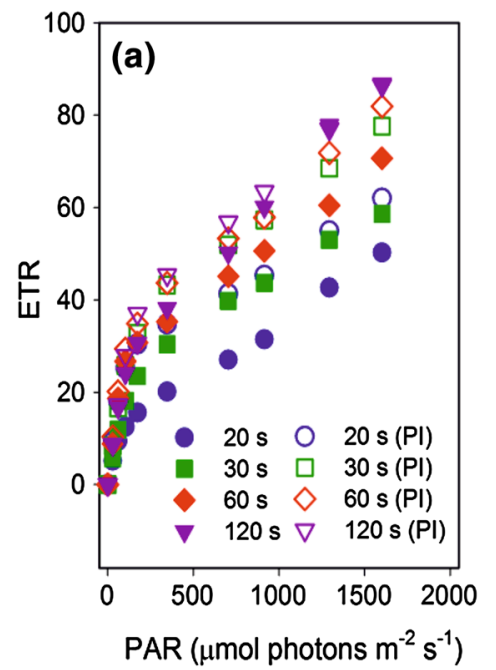

Fig. 6 Rapid light curves. a Example of RLCs (PAR vs. ETR) for which the duration of light intervals $(20,30,60,120 \mathrm{~s})$ had been varied. Closed symbols represent the values measured after $30 \mathrm{~min}$ dark acclimation (without pre-illumination), and open symbols represent values measured following $30 \mathrm{~min}$ of dark acclimation and $5 \mathrm{~min}$ of pre-illumination at a moderate light intensity $(100 \mu \mathrm{mol}$ photons $\mathrm{m}^{-2} \mathrm{~s}^{-1}$ ). b The ETR/ETRmax ratio (ETRmax represents the
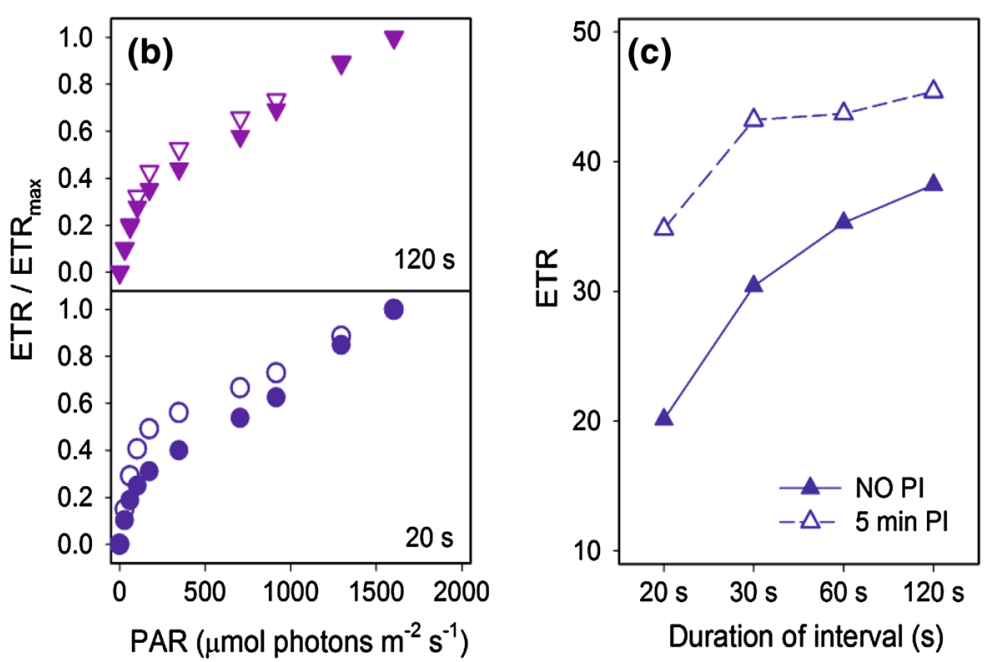

maximum value for each curve) of measurements with light intervals of 120 and $20 \mathrm{~s}$. c ETR values of experiments without preillumination (NO PI) and with 5 min of pre-illumination (5 min PI, $350 \mu \mathrm{mol}$ photons $\mathrm{m}^{-2} \mathrm{~s}^{-1}$ ). Measurements were made on Citrus leaves using a Dual-PAM fluorometer (Walz, Germany) (Brestič and Zivčak, unpublished data) 
induction. Thus, in pre-illuminated samples, a 30-s interval is sufficient to obtain appropriate values and shapes of the curves that are comparable to those measured with 2-min intervals (Fig. 6c).

RLCs have frequently been used in studies dealing with plant stress (reviewed in Brestic and Zivcak 2013). The value of the RLC approach increases if a second technique, e.g., $820 \mathrm{~nm}$ or gas exchange measurements, is applied simultaneously, or if fluorescence-imaging measurements are also made.

\section{Question 19. What is the JIP test?}

The idea that the fluorescence rise OJIP contains a lot of information on the photosynthetic system is already quite old. OJIP transients have been compared to a bar code for photosynthesis (Tyystjärvi et al. 1999) and extensive attempts to simulate OJIP transients have been made (see Lazár and Schansker (2009) for a review of these efforts). In 1991, Strasser and Govindjee published an article on the recording of the full fluorescence rise kinetics OJIP between $40 \mu \mathrm{s}$ and $1 \mathrm{~s}$ using a PEA instrument (see Strasser et al. 1995 for details). Four years later, Strasser and Strasser (1995) proposed a method to analyze these OJIP transients that was centered on the J-step [observed after 2-3 ms of strong illumination and equivalent to the $I_{1}$ step of Schreiber (1986)], which they called the JIP test (see Fig. 7).

The theoretical basis of the JIP test has been described in detail by Strasser et al. (2004). In the JIP test, OJIP transients are used to make a flux analysis, i.e., an analysis of the fate of photons absorbed by the PSII antennae

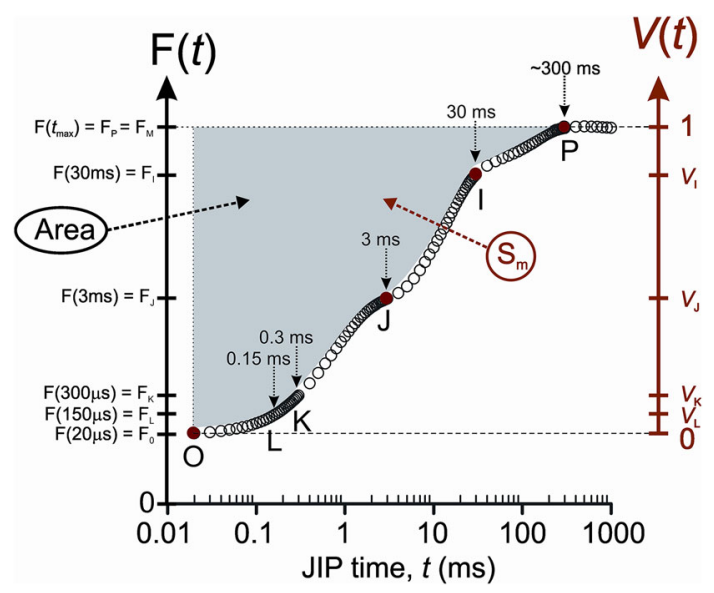

Fig. 7 Time points and parameters used in the JIP test. On the left hand side, the unnormalized $F$ scale associated with the complementary "Area" and on the right hand side, the $V$ scale double normalized between $\mathrm{O}$ and $\mathrm{P}$ associated with the normalized area Sm (Goltsev, unpublished data) (trapping, forward electron transport beyond $Q_{\mathrm{A}}$ and dissipation as heat). In the JIP test, the J-step is taken as the border between single and multiple turnovers. If we define multiple turnovers here as events related to electron transport beyond PSII, then this claim still remains valid. The JIP test depends strongly on the assumption that the $F_{\mathrm{O}^{-}}$-to- $F_{\mathrm{M}}$ rise reflects the reduction of $Q_{\mathrm{A}}$. The concept is internally consistent but the theoretical foundation of the interpretation of the parameters disappears the moment that this assumption turns out to be wrong (see Schansker et al. 2011, 2014 for a discussion of this point). An alternative approach to the interpretation of the OJIP transients is a classical physiological characterization of the various features of the fluorescence rise.

In the JIP test, it is assumed that the relative position of the J-step between $F_{\mathrm{O}}$ and $F_{\mathrm{M}}$ (i.e., $V_{\mathrm{J}}$, giving rise to the JIP-parameter $1-V_{\mathrm{J}}$ or $\Psi_{\mathrm{O}}$ ) gives information on photosynthetic electron transport beyond $Q_{\mathrm{A}}$ (e.g., Strasser et al. 1995, 2004). A physiological characterization of this feature, on the other hand, suggests that the parameter $V_{\mathrm{J}}$ depends on the redox state of the PQ-pool in darkness (Tóth et al. 2007a) and, under certain stress conditions, may also be affected by other factors, possibly the extent of stacking of the thylakoid membranes. In this case, electron transport beyond $Q_{\mathrm{A}}$ means a slowdown of the re-oxidation of $Q_{\mathrm{A}}^{-}$as the PQ-pool becomes more reduced, and fewer PQ molecules are bound to the $Q_{\mathrm{B}}$-site. Changes in $\Psi_{\mathrm{O}}$ may certainly point to stress.

In the JIP test, the parameters $F_{\mathrm{O}}$ and $F_{\mathrm{M}}$ were suggested to be a measure for the absorption flux (i.e., the number of photons absorbed per unit of time) per cross section (Strasser et al. 1995, 2004). With respect to this interpretation, it may be noted that a characterization of the changes in the $F_{\mathrm{O}}$ and $F_{\mathrm{M}}$ levels as a function of the Chl content of leaves showed that they are nearly insensitive to changes in the leaf chlorophyll content as long as the antenna sizes of the RCs remain unaffected (Dinç et al. 2012). However, we note that this observation probably does not apply to dilute algal and thylakoid suspensions.

Malkin (1966) and Murata et al. (1966) showed that the complementary area between the fluorescence transient and $F_{\mathrm{M}}$ in the presence of DCMU is proportional to the population of reduced $Q_{\mathrm{A}}$ molecules. In the JIP test, this principle is extended to the situation in the absence of DCMU, where the area between the fluorescence transient and $F_{\mathrm{J}}$ is assumed to equate one charge separation in all RCs, i.e., one electron transported, to which the total area above the OJIP transient can be normalized (see e.g., Strasser et al. 2004). Schansker et al. (2011, 2014) support and explain the relationship between the area above the OJIP transients (see Fig. 7) and the number of electrons that must be transported through the ETC before $F_{\mathrm{M}}$ is reached. 
In the JIP test, it is assumed that the slope taken between $F_{\mathrm{O}}$ and $F_{150 \mu \mathrm{s}}$ is sensitive to a phenomenon called "connectivity," i.e., the energy transfer between the antennae of several PSII RCs, whereas the slope taken between $F_{\mathrm{O}}$ and $F_{300 \mu \mathrm{s}}$ is insensitive to connectivity (Strasser and Stirbet 2001; and see Stirbet 2013 for a more in-depth discussion of connectivity in the absence of PSII inhibitors like DCMU).

The performance index [PI(ABS)] was introduced as an attempt to catch three different aspects of the photosynthetic activity of PSII in a single parameter (see Clark et al. 2000 for an early application of this parameter). PI(ABS) is the product of a parameter sensitive to the effective antenna size, a parameter based on the primary quantum yield of PSII and a parameter sensitive to changes in the relative position of $F_{\mathrm{J}}$. It is defined as:

$\mathrm{PI}(\mathrm{ABS})=\frac{\frac{F_{\mathrm{V}}}{F_{\mathrm{M}}} V_{\mathrm{J}}}{\frac{4\left(F_{270 \mu s}-F_{\mathrm{O}}\right)}{F_{\mathrm{M}}-F_{\mathrm{O}}}} \frac{\frac{F_{\mathrm{V}}}{F_{\mathrm{M}}}}{1-\frac{F_{\mathrm{V}}}{F_{\mathrm{M}}}} \frac{1-V_{\mathrm{J}}}{V_{\mathrm{J}}}$

with $\left.V_{\mathrm{J}}=\left(F_{\mathrm{J}}-F_{\mathrm{O}}\right) / \mathrm{F}_{\mathrm{M}}-F_{\mathrm{O}}\right)$. It is another JIP test parameter that has been shown to correlate with other stress parameters under a series of conditions (e.g., Clark et al. 2000; Misra et al. 2001a, b; Oukarroum et al. 2006). Physiological studies have further shown that the IP phase of the fluorescence rise is related to electron transport through PSI (Kautsky et al. 1960; Munday and Govindjee 1969; Schansker et al. 2005) and that the (relative) amplitude of the IP phase is linked to the PSI content of the leaf (Oukarroum et al. 2009; Ceppi et al. 2012). The JIP test approach remains a good and fast way to screen a large number of samples (Kalaji et al. 2011a, b). However, once parameters that correlate with certain features of a stress have been identified, it should not be blindly assumed that the interpretation of these parameters as given by the JIP test is correct (see also Stirbet and Govindjee 2011 for a discussion of this topic). In addition, it should be kept in mind that the JIP test depends strongly on normalizations which are very sensitive to the correctness of the determined $F_{\mathrm{O}}$ and $F_{\mathrm{M}}$ values. For example, in the case of heat stress, it is not easy to determine the $F_{\mathrm{O}}$ and $F_{\mathrm{M}}$ values correctly (see Tóth et al. 2007b).

\section{Question 20. What kind of values may one expect for particular fluorescence parameters?}

The $F_{\mathrm{V}} / F_{\mathrm{M}}$ values of plant species average approximately 0.83-0.84 in C3 plants under optimal conditions (Björkman and Demmig 1987; Pfündel 1998) and 0.78 in C4 plants (Pfündel 1998). Somewhat higher values have been described in certain broadleaved species. Lower values, on the other hand, are common in algae and lichens (see Trissl and Wilhelm 1993 for a discussion of these values). Stress conditions (e.g., photoinhibition) can significantly reduce these values (e.g., Björkman and Demmig 1987; Van Wijk and Krause 1991; Tyystjärvi and Aro 1996).

Photochemical quenching $\mathrm{qP}$, non-photochemical quenching defined as $\mathrm{qN}\left[=1-\left(F_{\mathrm{M}}{ }^{\prime}-F_{\mathrm{O}}{ }^{\prime}\right) /\left(F_{\mathrm{M}}-F_{\mathrm{O}}\right)\right]$, and the PSII operating efficiency in the light $\left(\Phi_{\text {PSII }}\right)$ can vary between 0 and 1 (see Question 14 for definitions of qP and $\left.\Phi_{\mathrm{PSII}}\right)$. The theoretical range for the values of the nonphotochemical quenching parameter NPQ $\left[=F_{\mathrm{M}} / F_{\mathrm{M}^{\prime}}-1\right]$ is from zero to infinity, but in most cases, it gives values between 0 and approximately 10 . However, NPQ values higher than 10 have been reported in bryophytes from sunexposed habitats (Marschall and Proctor 2004; see Buschmann 1999 for a discussion and comparison of $\mathrm{qN}$ and NPQ). High $\Phi_{\text {PSII }}$ values indicate that a large proportion of the light absorbed by the chlorophylls of the PSII antenna is converted into photochemical energy. At its upper limit, $\Phi_{\text {PSII }}$ could reach a value of 1 , which would mean that all absorbed energy is used for stable charge separations in PSIIs. From a practical point of view, this cannot be the case, due to the fundamental inefficiency of PSII (triplet formation, a small probability of fluorescence, and heat emission on each transfer of excitation energy between chlorophylls), and the contribution of fluorescence emitted by PSI has also an effect on the calculation (see Question 3 ). Therefore, $\Phi_{\mathrm{PSII}}$ can vary between zero and the $F_{\mathrm{V}} / F_{\mathrm{M}}$ value, which in $\mathrm{C} 3$ plants is about $0.83-0.85$, in $\mathrm{C} 4$ plants around 0.78 and in algae often below 0.7 (Pfündel 1998; Trissl and Wilhelm 1993). qP values near zero indicate that most of the PSII RCs are closed, and their $Q_{\mathrm{A}}$ is in the reduced state. Values near 1 indicate that $Q_{\mathrm{A}}$ is in the oxidized state, and almost all of the PSII centers are open for photochemistry. The non-photochemical quenching coefficients qN and NPQ are assumed to be zero in the dark-adapted state, because then $F_{\mathrm{V}}{ }^{\prime}=F_{\mathrm{V}}$ and $F_{\mathrm{M}}{ }^{\prime}=F_{\mathrm{M}}$. However, in some cases, positive values of these coefficients can also occur in darkness (see Question 17).

In higher plants, the induction kinetics of non-photochemical quenching triggered by high light usually have a typical time dependence: they increase during the first minute of illumination due to initiation of electron transport and $\Delta \mathrm{pH}$ formation preceding the activation of ATP synthase (e.g., Nilkens et al. 2010) and decrease again once the Calvin-Benson cycle is activated. This quenching is sensitive to the balance between the electron transport rate and its associated proton transfer toward the thylakoid lumen on the one hand and the rate of ATP synthesis and the associated release of protons from the thylakoid lumen on the other hand. This form of quenching (corresponding to $\mathrm{qE}$ quenching, see Question 15) relaxes quickly as soon as electron transport stops, e.g., as soon as the light is turned off (see e.g., Nilkens et al. 2010). Other processes 
contributing to NPQ have slower induction kinetics (see Questions 2.3 and 15) whose induction (e.g., photoinhibition) depends as well on light intensity. Higher non-photochemical quenching values related to higher values of $\mathrm{qE}$ under steady state conditions suggest a stronger imbalance between photosynthetic electron transport and the utilization of NADPH (reflected by lower qP values) (see e.g., Walters and Horton 1993). Under continuous and/or extreme stress, non-photochemical quenching can attain low values. This may in part be due to a loss of RCs. Photoinhibited PSII RCs lose their variable fluorescence, and as a consequence, this variable fluorescence can then no longer be quenched, which means less NPQ (Schansker and Van Rensen 1999). Low values may also be caused by decreased rates of linear electron transport generating a smaller transthylakoid proton gradient or to an increased permeability of the membrane due to lipid peroxidation caused by oxygen radicals, which will also reduce the build up of a $\Delta \mathrm{pH}$ over the membrane.

Deviations from the NPQ induction kinetics have been described in some green algae, where the NPQ induction capacity varies strongly depending on the species (see e.g., Bonente et al. 2008). For example, in Ulva laetevirens, NPQ was induced with an early peak within the first minute of exposure to high light, followed by a decrease and a subsequent rise (Bonente et al. 2008).

\section{Question 21. Which assumptions are made when interpreting fluorescence transient measurements?}

Both the quenching analysis and the JIP test (see Questions 15 and 19 for a discussion) are based on assumptions that were commonly made in the 1990s (e.g., van Kooten and Snel 1990 for the quenching analysis, Strasser 1996 for the JIP test and see also Stirbet and Govindjee 2011 for a list of assumptions). The most important assumption is that the fluorescence increase from $F_{\mathrm{O}}$ to $F_{\mathrm{M}}$ reflects mainly the reduction of $Q_{\mathrm{A}}$. This idea was first put forward by Duysens and Sweers (1963). However, this assumption was challenged almost from the beginning (see e.g., Delosme 1967). Delosme (1967) proposed the existence of two processes determining the fluorescence rise. His suggestion that the redox state of the PQ-pool could play a role (Delosme 1971) led to the idea that the $Q_{\mathrm{B}}$-site occupancy state was the second factor (see Samson et al. 1999); an idea that was extended further by Schansker et al. (2011) who suggested that the $Q_{\mathrm{B}}$-site occupancy state controlled the re-oxidation rate of $Q_{\mathrm{A}}^{-}$and who proposed on the basis of this idea that in the presence of $Q_{\mathrm{A}}^{-}$further excitations could induce conformational changes in the PSII RCs which would then cause an increase of the fluorescence yield. Considering the occupancy state idea, Schreiber (2002) proposed that the thermal phase might be explained by a reduction of the inactive branch of PSII. Vredenberg and co-workers (Vredenberg 2000; Vredenberg et al. 2006) developed another interpretation model, in which, in addition to $Q_{\mathrm{A}}^{-}$, the IP phase is determined by the electric field, and JI rise reflects an inactivation of PSII RCs (associated with proton transport over the membrane) in which $\mathrm{Pheo}^{-}$can accumulate. These alternative interpretations were challenged by Stirbet and Govindjee (2012). The first assumption that the $F_{\mathrm{O}}$-to- $F_{\mathrm{M}}$ rise is a reflection of the reduction of $Q_{\mathrm{A}}$ implies that it should always be possible to reach $F_{\mathrm{M}}$, since all $Q_{\mathrm{A}}$ can be reduced if the light intensity is high enough (i.e., when the excitation rate is much higher than re-oxidation rate of $Q_{\mathrm{A}}^{-}$by forward electron transport and/or the exchange of $\mathrm{PQH}_{2}$ for PQ at the $Q_{\mathrm{B}}$-site). However, Schreiber (1986), Samson and Bruce (1996) and Schansker et al. $(2006,2008)$ showed in several ways that this is not the case.

A second, related, assumption is that there are no changes in non-photochemical quenching during a saturating pulse. Finally, a third assumption is that the parameters $F_{\mathrm{V}} / F_{\mathrm{M}}$ and $\Phi$ PSII are measures of the PSII quantum yield and that $\Phi$ PSII can be used to calculate the photosynthetic electron transport rate. For $\Phi$ PSII, this assumption has been partially verified experimentally, showing under several conditions a linear correlation between the calculated photosynthetic electron transport rate and the $\mathrm{CO}_{2}$ assimilation rate (Genty et al. 1989; Krall and Edwards 1992 and see Questions 29 and 30). We note that the meaning of the parameter $F_{\mathrm{V}} / F_{\mathrm{M}}$ has not been derived experimentally but is based on an analysis of socalled competitive rate equations (fluorescence emission competes with other processes like heat emission and photosynthesis) for the $F_{\mathrm{O}}$ and $F_{\mathrm{M}}$ states (Kitajima and Butler 1975; Kramer et al. 2004). This analysis is correct as long as the fluorescence rise between $F_{\mathrm{O}}$ and $F_{\mathrm{M}}$ is determined by the reduction of $Q_{\mathrm{A}}$ only (see Schansker et al. 2014 for a discussion of this point).

\section{Question 22. Are there naturally occurring fluorescence quenchers other than $Q_{\mathrm{A}}$ ?}

Another fluorescence quencher that has been described extensively is $\mathrm{P}_{68} 0^{+}$(Butler 1972; Zankel 1973; Shinkarev and Govindjee 1993; Steffen et al. 2005). The short lifetime of $\mathrm{P} 60^{+}$keeps the population of this quencher low under most conditions. Simulation work has shown that under high light conditions, the highest concentration should occur around the J-step (Lazár 2003), which was supported by experimental observations (Schansker et al. 2011). However, $\mathrm{P}_{680}{ }^{+}$quenching does not affect the $F_{\mathrm{O}}$ 
and $F_{\mathrm{M}}$ levels. Oxidized PQ molecules can also quench fluorescence, but only in isolated thylakoids and in PSIIenriched membranes (Vernotte et al. 1979; Kurreck et al. 2000; Tóth et al. 2005a) and not in leaves (Tóth et al. 2005a). Other quenchers such as $\mathrm{Car}^{+}$and $\mathrm{Chl}^{+}$have been proposed and shown to play a role at temperatures below $100 \mathrm{~K}$ (Schweitzer and Brudvig 1997) in the case of Chl $\mathrm{Z}_{\mathrm{Z}}^{+}$, an accessory chlorophyll molecule in the RC of PSII, or to have a very short lifetime at room temperature (Steffen et al. 2001) in the case of $\mathrm{Car}^{+}$. Neither of these quenchers seems to play a role in the fluorescence measurements discussed in this paper.

\section{Question 23. What is the difference between fluorescence emission spectra recorded at $77 \mathrm{~K}$ and those recorded at room temperature?}

In Question 2 Sect. 4, measurements of $77 \mathrm{~K}$ fluorescence emission spectra were introduced as a method to study PSII and PSI antennae. The recording of fluorescence emission spectra is much easier at room temperature. In this case, one dominant peak at $\sim 684 \mathrm{~nm}$ is recorded, which is attributed principally to fluorescence emission by the PSIIcore complex (including the core antennae CP47 and CP43) and further a shoulder at 710-740 nm corresponding to several fluorescence emission sources-particularly PSILHCI and several minor PSII bands (Fig. 8) (Franck et al. 2005; Krausz et al. 2005; Pancaldi et al. 2002). When the temperature is lowered, the $684 \mathrm{~nm}$ band is replaced by two bands, peaking at 685 and $695 \mathrm{~nm}$, respectively; bands that in first instance were shown to be associated with the PSII core (Gasanov et al. 1979; Rijgersberg et al. 1979). The $695 \mathrm{~nm}$ band is due to fluorescence emission from CP47, whereas the $685 \mathrm{~nm}$ has been associated with fluorescence emission by CP43 [(Nakatani et al. 1984; for spectroscopic analyses of CP47 and CP43: see Alfonso et al. 1994 (for both); van Dorssen et al. 1987 (CP47); Groot et al. 1999 (CP43)]. Srivastava et al. (1999) showed with an experiment on greening of peas how the $695 \mathrm{~nm}$ band increases in intensity as the PSII antenna size increases. In other words, despite CP47 being the source of the $695 \mathrm{~nm}$ emission, it is sensitive to the number of LHCII subunits bound to PSII. The relationship between the antenna size of PSII and the amplitude of the $695 \mathrm{~nm}$ band is further strengthened by the observation that chloroplast samples frozen in the presence of a $\Delta \mathrm{pH}$ show a quenching of the $695 \mathrm{~nm}$ band (Krause et al. 1983). Based on a comparative study of photosynthetic mutants of Chlamydomonas reinhardtii, a relationship between LHCII-PSII association and emission intensity at $\sim 695 \mathrm{~nm}$ has also been proposed at room temperature (Ferroni et al. 2011). To detect fluorescence emitted by LHCII itself as an individual peak at $680 \mathrm{~nm}$, it is necessary to freeze the sample further to $4 \mathrm{~K}$ (see Govindjee 1995). However, a more or less distinct shoulder at $680 \mathrm{~nm}$ is often reported also at $77 \mathrm{~K}$ and attributed to the free LHCII trimers not linked with PSII in a stable association (Hemelrijk et al. 1992; Siffel and Braunova 1999; van der Weij-de Wit et al. 2007; Pantaleoni et al. 2009; Ferroni et al. 2013). At room temperature, the emission region around $680 \mathrm{~nm}$, never visible as an individual peak in the spectrum, was also assigned to a contribution by free LHCII (Ferroni et al. 2011). Strasser and Butler (1976) showed that the strong band at $730 \mathrm{~nm}$ at $77 \mathrm{~K}$ is in part caused by energy transfer from PSII to PSI. Weis (1985) demonstrated that the absorption of PSII fluorescence emission by PSI can be reduced considerably using diluted "leaf powder" instead of whole leaf fragments. When using liquid samples, such as microalgae suspensions or isolated thylakoids, the PSI re-absorption of emitted light can be reduced by an adequate dilution of the sample. The re-absorption phenomenon also affects room temperature spectra, resulting in a relative increase in the emission at $710-740 \mathrm{~nm}$ and in a red shift of PSII emission (Franck et al. 2002).

Room temperature fluorescence emission spectra are not frequently used for photosynthesis studies, because the spectral components are not as well characterized as the $77 \mathrm{~K}$ spectra are (Franck et al. 2002; Ferroni et al. 2011). However, methods have been developed to resolve at room temperature the contribution of PSII and PSI to Chl $a$ fluorescence under $F_{\mathrm{O}}, F_{\mathrm{M}}$, and steady state conditions $\left(F_{\mathrm{t}}\right)$ (Franck et al. 2002, 2005). Figure 8 gives examples of two such applications. Room temperature fluorescence spectra have also been used to evaluate the response of photosynthetic organisms (microalgae and in higher plants) to some environmental stresses (Romanowska-Duda et al. 2005, 2010; Ferroni et al. 2007; Baldisserotto et al. 2010, 2012; Burling et al. 2011; Hunsche et al. 2011). Finally, such spectra have been used as well to characterize developmental aspects of the photosynthetic membrane (Pancaldi et al. 2002; Baldisserotto et al. 2005; Ferroni et al. 2009, 2013) and, as discussed in Question 25, to estimate leaf chlorophyll content.

\section{Question 24. Are the fluorescence rise kinetics sensitive to the chlorophyll content of the leaf?}

For dilute solutions of chlorophyll molecules, the measured fluorescence intensity is proportional to the quantum yield of fluorescence multiplied by the number of photons absorbed and the chlorophyll concentration (Lakowicz 2009). On this basis, one would expect that the fluorescence intensity emitted by a leaf depends on the chlorophyll content of that leaf. However, as described under 
Question 4, the leaf is complex in optical terms, and it is difficult to predict if this physical law is really critical in determining the relationship between the chlorophyll content of the leaf and the fluorescence emission. Several experimental studies have addressed this question. Hsu and Leu (2003) showed that two leaves placed on top of each other emitted more Chl $a$ fluorescence than a single leaf. However, this is a quite artificial construct, and it can easily be shown that the outcome of the experiment strongly depends on the way the leaves were oriented (e.g., both adaxial sides up, or adaxial side up for the top leaf and the abaxial side for the bottom leaf) (Ceppi and Schansker, unpublished observations, 2008). Sušila et al. (2004) attempted to show an effect of chlorophyll content using thylakoid suspensions differing in their chlorophyll content. Thylakoid suspensions are homogeneous in their properties, whereas under natural conditions, a change in the chlorophyll content will be accompanied by an adaptation (change in antenna sizes and/or changes in PSI:PSII ratio) of the individual chloroplasts inside the leaf to their new light environment (see Question 4). To address the effect of changes in the chlorophyll content of a leaf on the measured fluorescence properties, it is important to find a natural system in which the leaves can acclimate to the effects of the changing chlorophyll content. Sugar beet plants grown hydroponically in the absence of magnesium or low sulfate concentrations show a gradual loss of chlorophyll; the activity of the remaining ETCs remains largely unaffected, and there were no overall changes in the antenna size (effect on $\mathrm{Chl} a / b$ ratio was small). Under these conditions, an up to fivefold decrease in the chlorophyll content left the $F_{\mathrm{O}}$ and $F_{\mathrm{M}}$ values unchanged and had only a marginal effect on the fluorescence rise kinetics (Dinç et al. 2012). On the other hand, changes in the PSII
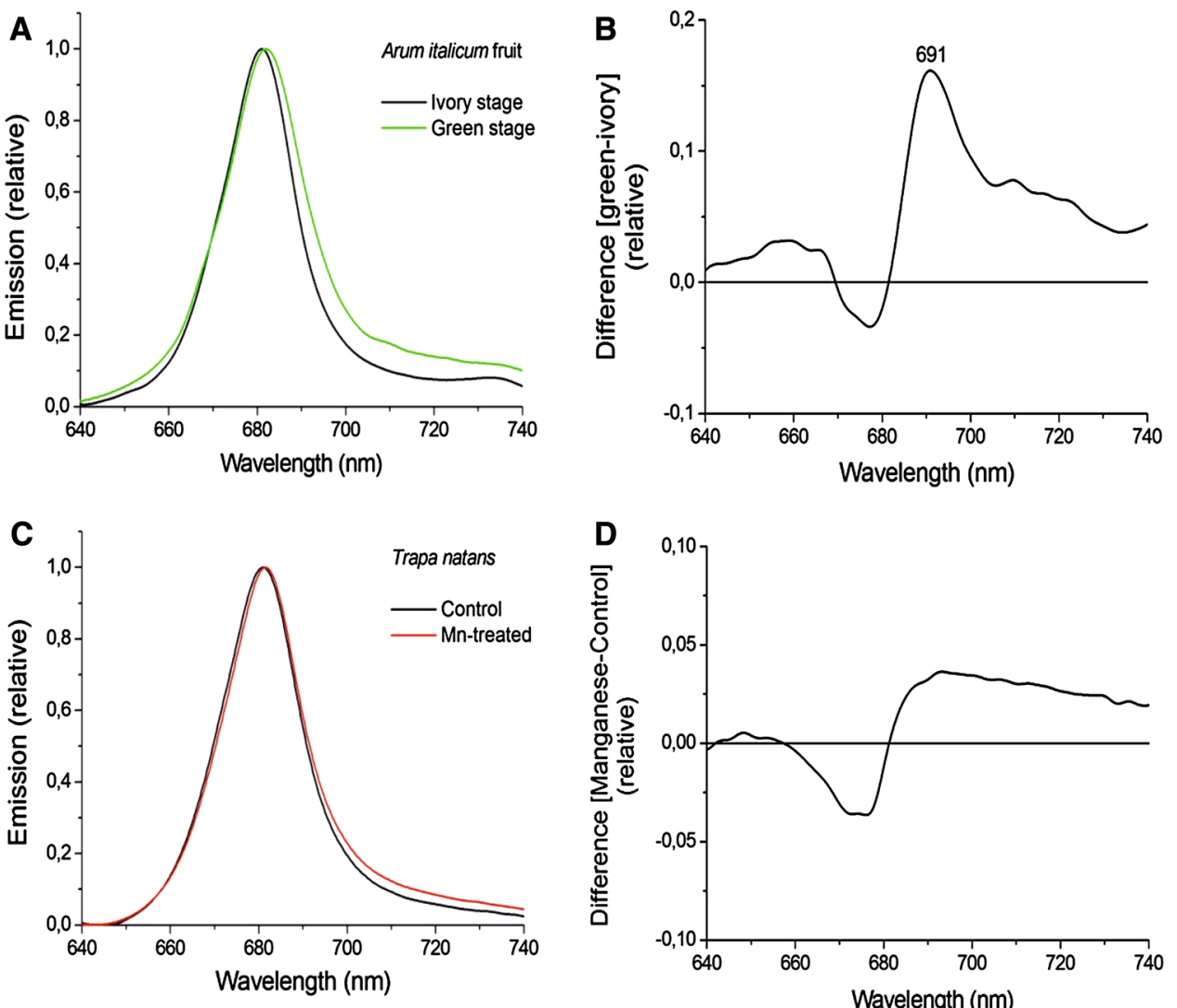

Fig. 8 Examples of applications of room temperature (RT) fluorescence emission spectra. a, b RT spectra of two developmental stages of chloroplasts of the fruit of Arum italicum. In its early stage of development (ivory stage), the fruit contains a rudimentary thylakoid system in amyloplasts which upon maturation are converted to chloroplasts (green stage; see Bonora et al. 2000). A difference spectrum (normalized green stage-normalized ivory stage) b shows that a distinctive trait of the amyloplast-to-chloroplast transition is the gain in emission at around $691 \mathrm{~nm}$, roughly corresponding to a PSIIcore contribution. An in-depth analysis of spectra in this system showed that the F695/F680 fluorescence ratio undergoes changes

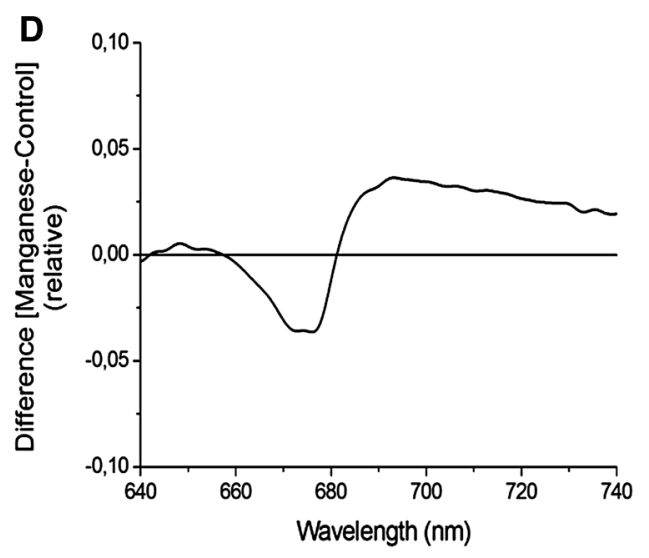

parallel to $F_{\mathrm{V}} / F_{\mathrm{M}}$, assembly of LHCII-PSII supercomplexes, and carbon fixation (Ferroni et al. 2013). c, d RT spectra to improve the description of chloroplast responses to stress. In the example, spectra were recorded from leaves of the aquatic plant Trapa natans, which were treated or not with manganese. In this species, acclimation to manganese includes an accumulation of LHCII in the leaf chloroplasts (Baldisserotto et al. 2013). Increased RT emission at long wavelength, as shown in the difference spectrum (d), points to the occurrence in vivo of uncoupled aggregates of LHCII which contribute fluorescence at around $700 \mathrm{~nm}$ (Ferroni and Pancaldi, unpublished data) 
antenna size did have an effect on the $F_{\mathrm{M}^{-} \text {-intensity (Dinç }}$ et al. 2012). In conclusion, there is little indication that a stress-induced Chl loss in leaves would complicate the interpretation of $\mathrm{Chl} a$ fluorescence measurements.

\section{Question 25. Can the leaf chlorophyll content be measured using fluorescence?}

Chlorophyll fluorescence emission spectra can be used to determine the chlorophyll content of green plants (Buschmann 2007). The ratio between chlorophyll fluorescence at $735 \mathrm{~nm}$ and that at $700 \mathrm{~nm}$ (F735/F700) is linearly proportional to chlorophyll content (Gitelson et al. 1999). Conversely, as discussed in Question 24, the $F_{\mathrm{M}}$ and $F_{\mathrm{O}}$ values are not related to the chlorophyll content in leaves (Dinç et al. 2012). It may also be noted that there are simple chlorophyll meters on the market (CL-01, Hansatech Instruments, UK; SPAD meter, Minolta, Japan; CCM200, Opti-Sciences, USA) that can be used to follow changes in the leaf chlorophyll content (see e.g., Cassol et al. 2008; Dinç et al. 2012). These measurements can then be calibrated against measurements of the chlorophyll extracted from leaf areas measured before with the chlorophyll meter (see e.g., Dinç et al. 2012). Chl measurements on dark-adapted leaves seem to give more reproducible results than measurements made on lightadapted leaves (Ceppi and Schansker, unpublished data, 2008). If the chlorophyll meter is used over the day on the same leaf, the readings change (Mishra, unpublished data, 2010), e.g., due to chloroplast movements, which change the absorbance properties of the leaf (see Wada 2013 for a review on chloroplast movements). Chloroplasts are known to re-arrange themselves inside the cell in response to the ambient blue light intensity, adapting the absorbance properties of the leaf to the circumstances (Sakai et al. 2001; Kasahara et al. 2002). This does not only affect chlorophyll meter measurements, but also normal fluorescence measurements (Brugnoli and Björkman 1992).

In practice, values measured using a $\mathrm{Chl}$ meter are often used as indicators for relative $\mathrm{Chl}$ changes. In that case, we assume that the measured values are a linear function of the leaf chlorophyll content between zero and the value measured on control leaves. However, in that case, it is important to test the validity of this assumption for each plant species and for each stress studied (Mishra, unpublished data, 2013).

\section{Question 26. Is it possible to compare different leaves?}

It is easy to take randomly two leaves from two plants of the same species and to make a fluorescence measurement.
But is it truly possible to compare these two measurements? It is likely that a difference in maximum fluorescence amplitude will be observed. Especially, when studying OJIP transients, the kinetics are often more interesting than the absolute amplitude, and in that case, the difference in the fluorescence amplitude is eliminated by double normalization between $F_{\mathrm{O}}$ and $F_{\mathrm{M}}$. Arithmetically, this is done in the following way: $\left(F_{\mathrm{t}}-\mathrm{F}_{\mathrm{O}}\right) /\left(F_{\mathrm{M}}-\right.$ $F_{\mathrm{O}}$ ). The effect of this calculation is to rescale each fluorescence value in a range going from 0 (corresponding to $F_{\mathrm{O}}$ ) to 1 (corresponding to $F_{\mathrm{M}}$ ). For a comparison of the kinetics of the individual rise phases of the OJIP transient, the same approach can be used. To compare the kinetics of the OJ-rise, the measured transient can be double normalized between $\mathrm{O}$ and $\mathrm{J}$ [i.e., $\left.\left(F_{\mathrm{t}}-F_{\mathrm{O}}\right) /\left(F_{\mathrm{J}}-F_{\mathrm{O}}\right)\right]$. In terms of nomenclature, double normalizations turn $F$ values into so-called $V$ values, like $V_{\mathrm{J}}$, which is the double normalized $F_{\mathrm{J}}$ value (see Strasser et al. 2004).

An important source of variability between leaves is the development of stress symptoms. A common stress-related effect is chlorosis, and it has been argued that a change in the chlorophyll content of the leaf has an impact on the fluorescence kinetics and thereby invalidates the analysis (Hsu and Leu 2003; Susila et al. 2004) but as discussed in Question 24, this is not the case as long as chloroplasts can adapt to their new light environment. In addition, if the development of the stress effects is followed over time, the gradually changing fluorescence properties will help the interpretation of the data.

A comparison of leaf fluorescence measurements on stressed and unstressed plants in the field is hampered by the fact that such leaves are often acclimated to completely different light environments. It is important to realize that growth light intensity affects the stoichiometries and composition of many components of the photosynthetic membrane like the PSII to PSI ratio, the LHCII to PSII ratio, and the amount of PSII-LHCII supercomplexes (e.g., Leong and Anderson 1984a, b; Walters and Horton 1994; Dietzel et al. 2008; Wientjes et al. 2013). Therefore, it is of fundamental importance that the light environment (full sunlight, shade, deep shade) of leaves/plants to be compared has been adequately analyzed before the effect of a certain stress is addressed by fluorimetric techniques. Several papers illustrate this, e.g., stressed and unstressed plants were compared by van Heerden et al. (2007), whereas Zubek et al. (2009) compared leaves of plants with and without mycorrhiza, both ascribing the observed difference in the initial slope of the measured OJIP transients to an effect on the oxygen evolving complex of PSII. An alternative and more likely explanation-a difference in the effective antenna size between the samples due to differences in the growth light conditions-was not considered. 
In summary, comparing leaves that develop under similar light conditions is relatively easy; however, comparing leaves that were growing under different light regimes is fraught with complications and should be avoided.

\section{Question 27. Can measurements made with different instruments during a large-scale field survey be compared in absolute terms?}

It is important to be aware that the use of different instruments, even from the same company and the same type, may yield different results in absolute terms. The light source used for saturating pulses of modulated instruments may age over time reducing its light intensity. The strength of the red LEDs of HandyPEAs often differs between instruments. When comparing measurements made with different types of instruments, differences may also be due to the specific geometry of the measuring cell or to the use of light sources emitting at different wavelengths. It is possible to reduce these differences by determining the light intensity dependence of the parameters of interest and using these data to change settings in order to obtain comparable results. Differences in wavelengths of the exciting light may be impossible to correct for. Green light for example has been shown to probe deeper in the leaves than red light; blue light is even more efficiently absorbed than red light (Terashima et al. 2009).

An example of the phenomenon, described above, is a study in which the same leaves were measured with different HandyPEA instruments (Bussotti et al. 2011a) calibrated with identical settings (lamp intensity $=3,000$ $\mu \mathrm{mol}$ photons $\mathrm{m}^{-2} \mathrm{~s}^{-1}$, time $=1 \mathrm{~s}$, gain = 1). Both original and normalized transient curves were compared. Original curves differed consistently (both the extreme values of $F_{\mathrm{O}}$ and $F_{\mathrm{M}}$ showed a large range of variability), but the differences decreased consistently after normalization (double normalization between $F_{\mathrm{O}}$ and $F_{\mathrm{M}}$-see Question 26 for a definition). The parameter $F_{\mathrm{O}} / F_{\mathrm{M}}$ (parameter which is sensitive to changes in heat dissipation in the PSII antenna), as well as the normalized steps of OJIP transients- $J$ and I (fluorescence intensities at 2-3 and $30 \mathrm{~ms}$, respectively)_showed very little variability when comparing the measurements of the different instruments with a coefficient of variation $(\mathrm{CV}=\mathrm{SD} /$ Mean) ranging from 3 to $5 \%$. The parameter PIabs, which consists of the product of a parameter sensitive to the effective antenna size, a parameter based on the maximum quantum yield of PSII, and a parameter sensitive to changes in the relative position of $F_{\mathrm{J}}$ (see Question 19) showed a very high variability among instruments (PIabs showed a $\mathrm{CV}=30 \%$; Bussotti et al. 2011a). The high intrinsic variability of PIabs between instruments is due to the fact that this parameter is sensitive to the initial slope of the fluorescence rise and the relative position of the J-step, two factors that are both relatively sensitive to the light intensity of the beam. This high intrinsic variability makes the PIabs less useful for large, multi-instrument surveys.

In conclusion, in the case of small-scale experiments, it is always preferable to use the same instrument for all the measurements of an experiment.

\section{Question 28. How should a sampling campaign be organized for an ecosystem?}

Large-scale surveys should be carried out using a robust sampling design. Criteria and examples of such designs can be found in many statistical manuals and textbooks (see Elzinga et al. 2001). Here, we discuss some specific issues related to the assessment of fluorescence parameters.

Two problems widely discussed in the context of forest health monitoring (Luyssaert et al. 2002) and other ecosystems (Tuba et al. 2010) are intercalibration and harmonization. Here, "intercalibration" refers to procedures aimed at reducing the differences between instruments discussed in Question 27, and "harmonization" refers to the sampling strategy. The main issues are the variability of the leaf responses within the crown/canopy and the ecological scale of the investigation (assessment of the response of the whole tree/plant, or of a target population of leaves).

A complete representation of a plant should take into account the different levels, age, and position of leaves. This would be the approach of choice but would require a large number of samples, and this would be difficult to realize in large-scale sampling. Thus, normally only one or a few leaf positions (e.g., sun leaves in the upper part of the crown, south exposed leaves, flag leaves, or fully developed leaves) are considered, depending on the purpose of the survey.

The number of leaves to be sampled depends on the internal variability of the parameters of interest. The following formula can be used for this calculation:

$n=Z_{\alpha}^{2} s^{2} / B^{2}$

where $n$ is the sample size; $Z_{\alpha}$ is the standard normal coefficient ( $=1.96$ for a $95 \%$ confidence level); $s$ is the $\mathrm{SD} ; B$ is the desired precision level expressed as percent of the mean value (Elzinga et al. 2001; Gottardini et al. 2014). A recent study of boreal forests (Pollastrini et al. 2014) found that, in the higher external part of a crown of Betula pendula, the $\mathrm{CV}$ among different leaves was very low for $F_{\mathrm{V}} / F_{\mathrm{M}}(1.6 \%)$, and increased for the parameters related to the step $\mathrm{J}\left(1-V_{\mathrm{J}}, \quad \mathrm{CV}=7 \%\right)$ and the step I $\left(\Delta V_{\mathrm{IP}}=1-V_{\mathrm{I}}, \mathrm{CV}=14 \%\right)$. We mention here that this 
type of studies demonstrated that the IP phase, linked to the PSI content (Oukarroum et al. 2009; Ceppi et al. 2012), is quite sensitive to different types of stress; e.g., it decreased in response to ozone (Bussotti et al. 2011b) and nitrogen deprivation (Nikiforou and Manetas 2011), while it increased in response to high light conditions (Desotgiu et al. 2012).

In order to sample as many leaves as possible during a single day, sampling must be performed during the whole day and cannot be limited to specific hours. As a consequence, leaves are sampled under different conditions of short-term light acclimation and different extents of photoinhibition. To reduce the associated variability, it is necessary to allow the regulatory mechanisms induced by the ambient light to relax and to allow the leaves to recover from photoinhibition, which means a sufficient period of at least $4-5 \mathrm{~h}$ of dark acclimation at a constant temperature must be made before measurement. In addition, to avoid the onset of leaf senescence or the induction of other stress factors that can change the physiological state of the leaf during sampling and dark acclimation of the leaves, all fieldwork must be performed as fast as possible. Managing a large number of samples in a short time, e.g., 1,000 samples in one day, requires fast instruments/experimental protocols. OJIP transients need less than $1 \mathrm{~s}$ of illumination, and their analysis is best suited for this kind of application.

\section{Question 29. What additional information can be obtained from simultaneous measurements of $\mathrm{CO}_{2}$ exchange and chlorophyll fluorescence?}

Modern Infrared gas analyzers (IRGAs; such as the CIRAS-3, PP Systems and LI-COR 6400) allow gas exchange and fluorescence to be measured simultaneously. This combination can provide information about effects on the photosynthetic ETC, Calvin-Benson cycle activity, and diffusional limitations at the same time. Additionally, it is possible to determine chlorophyll fluorescence parameters under particular conditions (e.g., increasing $\mathrm{CO}_{2}$ concentrations or low $\mathrm{O}_{2}$ concentrations) to determine the maximum electron transport rate. In this way, effects of a certain treatment can be more precisely assigned to a particular process in the whole photosynthetic apparatus than the use of these techniques individually would allow (see e.g., Laisk and Loreto 1996; Laisk et al. 2005).

Three potential applications for simultaneous measurements have been proposed in the literature:

(i) Analysis of alternative sinks of electrons (e.g., Flexas et al. 1998; Bota et al. 2004). Discrepancies between the electron transport rate (ETR) and the net $\mathrm{CO}_{2}$ assimilation rate $\left(A_{\mathrm{n}}\right)$ are an indicator of the existence of alternative electron sinks. For example, an increased ETR $/ A_{n}$ ratio indicates the existence of other electron sinks (e.g., Mehler reaction, photorespiration, nitrate reduction) in competition with $\mathrm{CO}_{2}$ assimilation (e.g., Bota et al. 2004). An important cause for an increase in ETR/ $/ A_{\mathrm{n}}$ is photorespiration (e.g., Galmés et al. 2007). Comparing measurements made at $2 \% \mathrm{O}_{2}$ (suppression of photorespiration) with measurements made at $21 \% \mathrm{O}_{2}$ (ambient) allows a quantification of this process (Rosenqvist and van Kooten 2003).

(ii) Calculation of $\mathrm{CO}_{2}$ diffusion resistance/conductance in the mesophyll, which in bifacial leaves is formed by the palisade and spongiform tissues (von Caemmerer 2000). Mesophyll conductance is an important variable controlling $\mathrm{CO}_{2}$ diffusion to the carboxylation site of Rubisco. Several methods have been proposed to estimate mesophyll conductance in leaves (for a detailed description of these methods, see e.g., Warren 2006; Flexas et. al. 2008). One of these methods is based on IRGA measurements (measurements of $\mathrm{CO}_{2}$ assimilation, $A_{\mathrm{n}} / C_{\mathrm{i}}$ curves) and the electron transport rate from chlorophyll fluorescence (e.g., Flexas et al. 2006)—a detailed description of this method is available elsewhere (Loreto et al. 1992; Evans and Loreto 2000; Flexas et al. 2008).

(iii) Sink limitations in photosynthesis (Rosenqvist and van Kooten 2003). In a variation of point (i) above, simultaneous IRGA and chlorophyll fluorescence measurements made at low (2\% $\mathrm{O}_{2}$, which suppresses photorespiration in $\mathrm{C} 3$ plants), and ambient $\left(21 \% \mathrm{O}_{2}\right)$ oxygen concentrations can be used to estimate changes in source-sink relationships in leaves (Rosenqvist and van Kooten 2003). Under non-sink restrictions and $2 \%$ oxygen, the $\mathrm{CO}_{2}$ assimilation rate $\left(A_{\mathrm{n}}\right)$ should increase, and the ETR should remain the same. By contrast, if the leaf is sink-limited, lowering the oxygen concentration to $2 \%$ will not affect $A_{\mathrm{n}}$, whereas the ETR will decrease (down-regulation by final product).

\section{Question 30. Can the wavelength dependence of the quantum yield for $\mathrm{CO}_{2}$ fixation be predicted by measuring chlorophyll fluorescence?}

Emerson and Lewis (1943) observed that the quantum yield for $\mathrm{O}_{2}$ evolution is wavelength dependent and that it 
dropped off quickly at wavelengths longer than $700 \mathrm{~nm}$. Similar wavelength dependence is observed for $\Phi \mathrm{CO}_{2}$ (McCree 1972; Inada 1976; Hogewoning et al. 2012). Typically, photosynthetic rates are higher when a leaf is illuminated with light in the red region $(600-680 \mathrm{~nm}$ ), compared with an equal number of photons in the blue or the green regions of the light spectrum. Beyond $700 \mathrm{~nm}$ (i.e., the FR region), $\Phi \mathrm{CO}_{2}$ declines rapidly to nearly zero at about $730 \mathrm{~nm}$.

Genty et al. (1989) demonstrated that the PSII operating efficiency (i.e., $F_{\mathrm{q}}{ }^{\prime} / F_{\mathrm{M}}{ }^{\prime}$ or $\Phi_{\mathrm{PSII}}$ ) correlates linearly with $\Phi \mathrm{CO}_{2}$ if the photosynthetic steady state is induced by white light of different intensities, while photorespiratory activity is low. This is always the case in $\mathrm{C} 4$ plants and in $\mathrm{C} 3$ plants, this occurs when the $\mathrm{O}_{2}$ concentration is low (1-2 \%) (see also Question 29; Genty et al. 1989; Krall and Edwards 1992). In contrast to the relationship between $\Phi \mathrm{CO}_{2}$ and light intensity, $\mathrm{Chl} a$ fluorescence measurements are unsuitable for the estimation of the relationship between $\Phi \mathrm{CO}_{2}$ and the wavelength of irradiance used. To understand why, it is important to consider the factors that may affect the wavelength dependence of both $\Phi \mathrm{CO}_{2}$ and $\Phi_{\text {PSII. }}$

First, different wavelengths are not reflected and transmitted to the same extent by leaves. Hence, the fraction of light absorbed by a leaf is wavelength dependent (e.g., Vogelmann and Han 2000; see also Question 4). This also explains why most leaves are green and not, for example, black-relatively more green light is reflected and transmitted than red and blue light, and therefore, the fraction of red and blue light absorbed by a leaf is higher than the fraction of green light that is absorbed (Terashima et al. 2009). A lower fraction of incident light reaching the photosystems will directly result in a loss of $\Phi \mathrm{CO}_{2}$ on an incident light basis. However, at low light intensities in the linear part of the light-response curve, there are no limitations for the electron flow on the acceptor side of PSII. Therefore, within a range of low light intensities (typically between PPFD of 0 and $50 \mu \mathrm{mol}$ photons $\mathrm{m}^{-2} \mathrm{~s}^{-1}$, or an even narrower range for shade-leaves), $\Phi_{\text {PSII }}$ does not necessarily change as a result of small changes in the light intensity. Beyond this range of low light intensities, $\Phi_{\mathrm{PSII}}$ decreases when the light intensity increases, due to limitations for the electron flow on the acceptor side of PSII (see Question 2 Sect. 1 for electron transfer rates on the acceptor side of PSII). Thus, wavelength-dependent differences in the fraction of incident light reaching the photosystems are reflected by differences in $\Phi_{\mathrm{CO}_{2}}$, but at low light intensities not necessarily by differences in $\Phi_{\mathrm{PSII}}$.

Second, carotenoids differ in the efficiency (35-90 \%) with which they transfer excitation energy to chlorophylls, whereas the chlorophyll to chlorophyll energy transfer efficiency in antenna complexes is nearly $100 \%$ (Croce et al. 2001; de Weerd et al. 2003a, b; Caffarri et al. 2007). The transfer efficiency of carotenoids depends on their chemical structure and position within the photosynthetic apparatus. Carotenoids have absorption maxima in the blue and green regions, and therefore, blue light is used less efficiently by the photosystems than e.g., red light. Wavelength-dependent differences in the fraction of light absorbed by carotenoids affect the fraction of absorbed light reaching the RCs of the photosystems. This leads to the same argument as in the previous paragraph, i.e., this effect decreases $\Phi \mathrm{CO}_{2}$ but at low light intensities does not necessarily decrease $\Phi_{\mathrm{PSII}}$.

Third, leaves contain non-photosynthetic pigments such as flavonoids and free carotenoids. These pigments predominantly absorb light in the UV region but also in the blue and green part of the spectrum. These non-photosynthetic pigments are not connected to the photosystems and do not transfer the absorbed energy to the photosynthetic apparatus (see Question 31 for a discussion of these compounds and their detection). The absorption of light by nonphotosynthetic pigments will reduce the fraction of the incident light reaching the photosystems especially in the blue and to a smaller extent in the green. Again this will affect $\Phi \mathrm{CO}_{2}$ at these wavelengths but at low light intensities not necessarily $\Phi_{\text {PSII }}$.

Finally, the pigment composition and absorbance properties of PSI and PSII differ, and therefore, the balance of excitation between the two photosystems is wavelength dependent for a given state of the photosynthetic apparatus (e.g., Evans 1986; Chow et al. 1990a, b; Melis 1991; Walters and Horton 1995; Hogewoning et al. 2012). In practice, when light within a narrow-band wavelength range is used to illuminate a white-light acclimated leaf, one of the two photosystems is often excited more strongly than the other. Any imbalance in excitation between the two photosystems results in a loss of $\Phi \mathrm{CO}_{2}$. This wavelength dependence is especially clear in the FR region. FR light still quite efficiently excites PSI but is very inefficiently absorbed by PSII (see Question 16). This is called "the red drop" and, as noted above, this leads to a rapid decline of $\Phi \mathrm{O}_{2}$ and consequently of $\Phi \mathrm{CO}_{2}$ as well at wavelengths longer than $685 \mathrm{~nm}$. Obviously, when PSI is excited strongly by FR light, but PSII is excited only very weakly, electron flow from PSII to PSI is not restricted, and therefore, $\Phi_{\mathrm{PSII}}$ will be high. However, due to the inefficient absorption of the FR photons by PSII, linear electron flow is low, and therefore, $\Phi \mathrm{CO}_{2}$ is low for FR light. On the other hand, if PSII is excited more strongly than PSI, the consequent loss of $\Phi_{\mathrm{PSII}}$ is reflected by a proportional loss of $\Phi \mathrm{CO}_{2}$. Wavelengths in the range around $480 \mathrm{~nm}$ (blue) result in the strongest preferential excitation of PSII and therefore the strongest loss of both $\Phi \mathrm{CO}_{2}$ and $\Phi_{\mathrm{PSII}}$ (Hogewoning et al. 2012). However, $\Phi_{\text {PSII }}$ is also an unreliable 
measure of $\Phi \mathrm{CO}_{2}$ for these blue wavelengths, due to the absorption by carotenoids and non-photosynthetic pigments (see above).

In summary, $\Phi_{\text {PSII }}$ calculated from chlorophyll $a$ fluorescence measurements is an unsuitable parameter for estimating the wavelength dependence of $\Phi \mathrm{Co}_{2}$. Wavelength-dependent changes in (1) the absorbed light fraction, (2) the light fraction absorbed by photosynthetic carotenoids, and (3) the light fraction absorbed by non-photosynthetic pigments, directly affect the fraction of photons reaching the photosystems and therefore $\Phi \mathrm{Co}_{2}$. However, at low light intensities, changes in the fraction of photons reaching the photosystems may not affect $\Phi_{\text {PSII }}$. Furthermore, (4) some wavelengths preferentially excite PSI, resulting in high $\Phi_{\text {PSII }}$ values but low $\Phi \mathrm{CO}_{2}$ values. As a consequence, for a reliable measurement of the wavelength dependence of $\Phi \mathrm{Co}_{2}$, gas exchange measurements remain the gold standard.

\section{Question 31. Can anthocyanins and flavonols be detected by chlorophyll fluorescence?}

In vivo non-destructive determination of anthocyanins and flavonols in green parts of plants can be made using the fluorescence excitation ratio method (FER) (Bilger et al. 1997; Agati et al. 2011). The FER method is based on the measurement of chlorophyll fluorescence induced by different excitation wavelengths. The extent of absorbance of light by the epidermal polyphenols can be derived on the basis of the ratio of chlorophyll fluorescence emission intensities induced by a standard red beam and a UV-VIS beam (wavelengths strongly absorbed by epidermal polyphenols). The role of different anthocyanins and flavonols can be distinguished by choosing appropriate wavelengths based on the specific absorbance spectra of the different anthocyanins and flavonols.

The chlorophyll fluorescence excitation technique was originally developed to assess UV-absorbing compounds in the leaf epidermis (Bilger et al. 1997). Ounis et al. (2001) extended the method developing remote sensing equipment (dual excitation FLIDAR) to study polyphenols not only in leaves but also in canopies of trees. This method has also been used for the determination of the presence of flavonoids, including anthocyanins, in the skins of fruits like grapes (Kolb at al. 2003), apples (Hagen et al. 2006), and olives (Agati et al. 2005). Betemps et al. (2011) showed that in fruits, the anthocyanins and other flavonoids localized in the outer skin layers reduce the chlorophyll fluorescence signal in proportion to the concentration of these polyphenols.

Pfündel et al. (2007) investigated two different types of commercial portable UV fluorometers for in vivo screening of anthocyanins and carotenoids in leaves. The UV-APAM fluorometer (Walz, Germany) makes use of a blue reference beam, whereas the Dualex fluorometer (FORCEA, France) makes use of a red reference beam. For measurements on green leaves, the two instruments gave similar results, whereas the anthocyanins common in fruits absorbed part of the blue light of the UV-A-PAM reference beam which led, for fruits, to higher estimates for epidermal UV transmittance compared to that by the Dualex fluorometer. Pfündel et al. (2007) also noted that the absence of $\mathrm{Chl} b$ (e.g., in the barley chlorina $\mathrm{f} 2$ mutant) affected the determination of the polyphenols. Ben Ghozlen et al. (2010) developed and described an improved instrument, which they called the Multiplex (FORCE-A, France). It contains four light-emitting diodes (LEDs): UVA $(370 \mathrm{~nm})$, blue $(460 \mathrm{~nm})$, green $(515 \mathrm{~nm})$, and red $(637 \mathrm{~nm})$ and three diodes to detect fluorescence emission at 590, 685, and $735 \mathrm{~nm}$. The three diodes allow corrections for differences in the chlorophyll content of the sample. The red LED provides the reference beam, because it corresponds to a wavelength not absorbed by anthocyanins or flavonols. The fluorescence induced at this wavelength is compared with the fluorescence intensity induced by the excitation wavelength specific for the polyphenol of interest (e.g., green $515 \mathrm{~nm}$ light for anthocyanins or $370 \mathrm{~nm}$ UV-A light for flavonols). Ben Ghozlen et al. (2010) derived formulas to correlate these ratios with the actual polyphenol content of the sample.

In summary, a fluorescence-based method and accompanying equipment have been developed to determine the anthocyanin and flavonol content of leaves and fruits. In the case of fruits, the choice of the color (blue or red) of the reference beam influences the results, something that does not affect leaf measurements.

\section{Question 32. Can Chl $\boldsymbol{a}$ fluorescence be used as an indicator for a specific stress in plants?}

To use Chl $a$ fluorescence as a tool to identify a specific stress, the effects of that stress on the photosynthetic apparatus must be understood (Kalaji et al. 2012a, b). If heat stress destroys the donor side of part of the PSII RCs, it reduces the electron donation capacity of all PSII RCs together and, as a consequence, causes a slow down of the JI rise as measured by a PEA-type instrument (Srivastava et al. 1997 and see also Schreiber and Neubauer 1987). It also changes the recombination properties of the affected PSII RCs when measuring DF (Čajánek et al. 1998). In extreme cases, when all or nearly all PSII donor sides have been destroyed, the fluorescence rise levels off after $\sim 300 \mu$ s of illumination (i.e., one charge separation) and then declines; this fluorescence pattern is called the K-peak 
(Guissé et al. 1995; Srivastava et al. 1997; Lazár et al. 1997). UV radiation may also destroy the donor side of PSII (e.g., Ohnishi et al. 2005; Hakala et al. 2005), but, at the same time, may have additional affects on the PSII RC (e.g., Vass et al. 1996) and, thereby, on the fluorescence kinetics. For both drought stress and sulfate deficiency, it was shown that they affect PSI (Oukarroum et al. 2009; Ceppi et al. 2012). Again, a combination of experimental phenomena is needed to distinguish these stress conditions. Another complication is that the PSII to PSI ratio that affects the parameter $\Delta V_{\mathrm{IP}}$ is regulated by the growth light intensity and quality as well (Leong and Anderson 1984b; Lee and Whitmarsh 1989; Chow et al. 1990a, b). Finally, there are considerable kinetic differences between the OJIP transients obtained from different plant species (Kirova et al. 2009). This means that good references are needed to determine if something is a stress effect, taking into account the normal plasticity of the OJIP transients. The available physiological studies often concentrate on the effects of severe stress under laboratory conditions. In the field, milder stress effects are often observed, which possibly have to be distinguished from other sources of variability, so that additional research efforts will be needed to obtain reliable "fingerprints" for a particular stress. An example of the type of research needed is a study by Kalaji (2011) who characterized the effects of 16 abiotic stresses on the fluorescence properties of two Syrian landraces (cvs. Arabi Abiad and Arabi Aswad) of barley (see also Kalaji and Guo 2008).

Another approach is to make mathematical analyses of sets of OJIP transients in combination with DF and $820 \mathrm{~nm}$ transmission transients. Goltsev et al. (2012) trained an artificial neural network to estimate the relative water content (RWC) of leaves; they obtained a correlation value of $R^{2}=0.98$ between the estimated RWC value and the gravimetrically determined RWC value of the analyzed leaves.

In France, commercial software was developed that compares measured OJIP transients with a database of fluorescence transients measured on plants of dozens of genotypes of agricultural and horticultural crops suffering from deficiencies of the following elements: N, Fe, Mn, $\mathrm{Mg}, \mathrm{P}, \mathrm{S}, \mathrm{Ca}$, and $\mathrm{B}$. This approach has similarities with the one discussed above, but it is more ambitious in its scope. This software is at the moment very popular among farmers, especially in Poland, Ukraine, and Russia, where it is promoted by producers of fertilizer. Kalaji et al. (unpublished data, 2013) did many experiments to test the software and suggested analysis, comparing the fluorescence analysis with the chemical analysis of several plant species grown under different conditions of nutrient deficiency. These studies suggested that this method needs further improvements to achieve a general validity.
For the moment, it is not possible to identify specific stresses using Chl $a$ fluorescence. As noted above, different stresses may have similar effects on the photosynthetic system. In addition, in the field, plants are often subjected to several stresses at the same time, e.g., a combination of drought, high light, and heat stress. In the laboratory, it is possible to induce clear symptoms, whereas in the field, a combination of a less severe stress and acclimation may cause less specific symptoms. In other words, the complicated relationship between fluorescence kinetics, stress, and natural variation is not yet sufficiently well understood to use fluorescence measurements as fingerprints for specific stresses under natural conditions.

\section{Question 33. Is Chl $a$ fluorescence a useful tool for the monitoring of aquatic ecosystems?}

The use of Chl $a$ fluorescence measurements for the study of aquatic environments is a topic by itself, and here only a few points are made. This topic was reviewed in depth in a recent book edited by Suggett et al. (2011).

The estimation of biomass production in aquatic environments is one of the research topics in which fluorescence techniques have played a major role and for which special equipment was developed. Falkowski and Kolber (1990) developed a submersible pump-probe instrument (see Question 2 Sect. 1 for the principle) to study biomass productivity profiles along the water column in the ocean. Further, Kolber et al. (1998) discussed a new fluorescence approach, which they called the FRR approach which was originally developed for aquatic studies. Instead of continuous light, subsaturating excitation flashes (of which the spacing can be varied) are used to induce photosynthesis. With these flashlets, the authors could create STFs as well as multiple turnover pulses and, at the same time, study the dark relaxation kinetics of fluorescence. One of the parameters that could be determined was the effective PSII antenna cross section. Using a Xenon-PAM (Walz, Germany), Geel et al. (1997) studied several classes of aquatic organisms in order to derive the oxygen evolution activity of these organisms on the basis of fluorescence measurements. Kromkamp and Forster (2003) have reviewed such studies.

Another important difference between measurements on plants and measurements in an aquatic environment is that aquatic samples often consist of a mixture of photosynthetic organisms. To cope with this problem, several instruments were developed that make use of differences in the pigment composition of different classes of photosynthetic organisms. Schreiber (1998) has described an instrument built by Kolbowski and Schreiber called the PHYTO-PAM Phytoplankton analyzer (Walz, Germany). 
The instrument does not use a monochromatic modulated beam but excites the samples alternately with weak $10 \mu \mathrm{s}$ light pulses of $470,535,620$, and $650 \mathrm{~nm}$ (inducing $F_{\mathrm{O}}$ ) to distinguish between cyanobacteria, green algae, and diatoms. Deconvolution of the algal composition was possible using reference spectra derived from pure cultures of particular classes of organisms. In addition, the instrument allowed the estimation of the activity of these classes of organisms using saturating light pulses (see Questions 2.3, 10 , and 15).

Beutler et al. (2002) built a submergible instrument called bbe Fluoroprobe ${ }^{\mathrm{TM}}$ (Moldaenke, Germany) that made use of five excitation wavelengths $(450,525,570$, 590 , and $610 \mathrm{~nm}$ ) with which particular accessory pigments can be relatively specifically excited allowing the detection of peridinin containing dinoflagellates and Pyrrophyta, chlorophyll $b$ containing green algae, fucoxanthin containing diatoms, and zeaxanthin as well as phycobiliprotein containing cyanobacteria or cryptophycaea. Reference spectra were used to determine the chlorophyll content associated with each class. Rolland et al. (2010) using this equipment for a monitoring study of the Marne reservoir summarize its application in monitoring studies up till that time and note that it can be used down to $100 \mathrm{~m}$, and that it has a short response time.

Further, Schreiber et al. (2012) have developed a new Multi-Color-PAM (Walz, Germany) instrument that combines multi-spectral excitation (400, 440, 480, 540, 590, and $625 \mathrm{~nm}$ ) with the possibility to measure fast fluorescence kinetics as well as the absorption cross section of PSII antennae.

Photosynthetic aquatic organisms (including aquatic plants such as Spirodela) in combination with fluorescence measurements can also be used to monitor the presence of pesticides, heavy metals, and natural compounds that affect the photosynthetic apparatus. Snel et al. (1998) using a modulated PAM fluorometer and monitoring ETR followed the effect of low concentrations of linuron in microcosm experiments. Another example of the application of a PAM fluorometer was published by Perreault et al. (2010) who evaluated the effect of copper oxide nanoparticles on Lemna gibba using among other things the quenching analysis. Srivastava et al. (1998) using a PEA instrument showed that the cyanobacterial toxin fischerellin A caused an increase of $F_{\mathrm{J}}$; this indicates that fischerellin A affects the acceptor side of PSII like DCMU does. Bueno et al. (2004) showed an effect of lindane on the cyanobacterium Anabaena; they observed that this pesticide initially affects the amplitude of the JIP phase and after longer incubation times (12-24 h) causes a general suppression of the fluorescence intensity. In other studies, the effects of heavy metals like cadmium (Romanowska-Duda et al. 2005) or chromate (Susplugas et al. 2000) on Spirodela oligorrhiza have been studied. Finally, Chl $a$ fluorescence is also a useful tool for the study of hydrogen production in e.g., Chlamydomonas reinhardtii (see e.g., Antal et al. 2006)

\section{Concluding remarks}

For anyone who is beginning to use Chl $a$ fluorescence, the overwhelming number of studies that already has been carried out may make it difficult to quickly discover what is already known and which experiments will add something new to the literature. Even so, it is important to formulate first some questions that are worth answering. Two points are worth keeping in mind. In the first place, the "flash," "pulse," and "steady state" communities live often in parallel universes; as a consequence, there are still many opportunities for a more integrated use of these techniques. In the second place, the currently available fluorescence devices can do much more than the few standard protocols that are most frequently used.

As this educational review suggests, there are many aspects of fluorescence that can be studied with different devices best adapted for the study of these different aspects. Flash experiments can be used to study the electron transfer reactions within PSII, direct fluorescence measurements are best for the measurement of the OJIP transients, which follow the reduction of the photosynthetic electron chain, and modulated measurements are best for steady state photosynthesis and the study of light-induced regulatory mechanisms affecting the antenna of PSII. The power of fluorescence techniques can be increased considerably by simultaneously measuring other parameters, such as $820 \mathrm{~nm}$ transmittance changes (probing PSI) or $\mathrm{CO}_{2}$ assimilation.

There are only a few basic principles that determine the yield of fluorescence. However, due to the fact that it is sensitive to many processes that differ between photosynthetic organisms, light acclimation states, intactness of samples, and stress conditions, a myriad of responses has been documented in the literature. The fluorescence literature may often be confusing and contradictory, but it contains a wealth of data and observations that we all need to understand. Only in that way, the wealth of information generated by past fluorescence research can be maximally exploited.

The contributing authors are available to be contacted by researchers for further discussions on the application of $\mathrm{Chl}$ $a$ fluorescence through the following website: https://groups. google.com/forum/?hl=en\#!forum/chlorophyllfluorescence where they will provide regular feedback.

Acknowledgments The authors thank Govindjee (University of Illinois at Urbana-Champaign, USA) for his support, assistance, and 
helpful comments during the preparation of the manuscript. The authors are also grateful to Dr Giles Johnson (University of Manchester, UK), Peter Hooda (Kingston University, UK), Dr. Mahendra Rai (SGB Amravati University, India), Dr. Szilvia Z. Tóth (Biological Research Centre Szeged, Hungary), and Dr. Gerald E. Edwards (Washington State University, USA) for their valuable comments and suggestions to improve the quality of this paper. M.H. Kalaji acknowledges Prof Helmut Lichtenthaler, Dr Ulrich Schreiber, Dr Alexandra Stirbet, and Dr Dusan Lazár for their encouragement.

Open Access This article is distributed under the terms of the Creative Commons Attribution License which permits any use, distribution, and reproduction in any medium, provided the original author(s) and the source are credited.

\section{References}

Agati G, Pinelli P, Cortés Ebner S, Romani A, Cartelat A, Cerovic ZG (2005) Nondestructive evaluation of anthocyanins in olive (Olea europaea) fruits by in situ chlorophyll fluorescence spectroscopy. J Agric Food Chem 53:1354-1363

Agati G, Cerovic ZG, Pinelli P, Tattini M (2011) Light-induced accumulation of ortho-dihydroxylated flavonoids as non-destructively monitored by chlorophyll fluorescence excitation techniques. Environ Exp Bot 73:3-9

Alfonso M, Montoya G, Cases R, Rodriguez R, Picorel R (1994) Core Antenna complexes, CP43 and CP47, of higher plant photosystem II. Spectral properties, pigment stoichiometry, and amino acid composition. Biochemistry 33:10494-10500

Antal TK, Volgusheva AA, Kukarskih GP, Bulychev AA, Krendeleva TE, Rubin AB (2006) Effects of sulfur limitation on photosystem II functioning in Chlamydomonas reinhardtii as probed by chlorophyll $a$ fluorescence. Physiol Plant 128:360-367

Baker NR (2008) Chlorophyll fluorescence: a probe of photosynthesis in vivo. Annu Rev Plant Biol 59:89-113

Balachandran S, Osmond CB, Daley PF (1994) Diagnosis of the earliest strain-specific interactions between tobacco mosaic virus and chloroplasts of tobacco leaves in vivo by means of chlorophyll fluorescence imaging. Plant Physiol 104:1059-1065

Baldisserotto C, Ferroni L, Moro I, Fasulo MP, Pancaldi S (2005) Modulations of the thylakoid system in snow xanthophycean alga cultured in the dark for two months: comparison between microspectrofluorimetric responses and morphological aspects. Protoplasma 226:125-135

Baldisserotto C, Ferroni L, Zanzi C, Marchesini R, Pagnoni A, Pancaldi S (2010) Morpho-physiologcal and biochemical responses in the floating lamina of Trapa natans exposed to molybdenum. Protoplasma 240:83-97

Baldisserotto C, Ferroni L, Giovanardi M, Boccaletti L, Pantaleoni L, Pancaldi S (2012) Salinity promotes growth of freshwater Neochloris oleoabundans UTEX 1185 (Sphaeropleales, Chlorophyta): morphological aspects. Phycologia 51:700-710

Baldisserotto C, Ferroni L, Pantaleoni L, Pancaldi S (2013) Comparison of photosynthesis recovery dynamics in floating leaves of Trapa natans after inhibition by manganese or molybdenum: effects on photosystem II. Plant Physiol Biochem 70:387-395

Ballottari M, Girardon J, Dall'Osto L, Bassi R (2012) Evolution and functional properties of photosystem II light harvesting complexes in eukaryotes. Biochim Biophys Acta 1817:143-157

Bannister TT, Rice G (1968) Parallel time courses of oxygen evolution and chlorophyll fluorescence. Biochim Biophys Acta 162:555-580

Beardall J, Quigg A, Raven JA (2003) Oxygen consumption: photorespiration and chlororespiration. In: Larkum AW, Douglas
SE, Raven JA (eds) Photosynthesis in algae. Kluwer, Dordrecht, pp 157-181

Bellafiore S, Barneche F, Peltier G, Rochaix J-D (2005) State transitions and light adaptation require chloroplast thylakoid protein kinase STN7. Nature 433:892-895

Ben Ghozlen N, Cerovic ZG, Germain C, Toutain S, Latouche G (2010) Non-destructive optical monitoring of grape maturation by proximal sensing. Sensors 10:10040-10068

Bennoun P (1982) Evidence for a respiratory chain in the chloroplast. Proc Natl Acad Sci USA 79:4352-4356

Bennoun P (2002) The present model for chlororespiration. Photosynth Res 73:273-277

Berera R, van Grondelle R, Kennis JTM (2009) Ultrafast transient absorption spectroscopy: principles and application tot photosynthetic systems. Photosynth Res 101:105-118

Betemps DL, Fachinello JC, Galarca SP, Portela NM, Remorini D, Massai R, Agati G (2011) Non-destructive evaluation of ripening and quality traits in apples using a multiparametric fluorescence sensor. J Sci Food Agric 92:1855-1864

Beutler $\mathrm{M}$, Wiltshire $\mathrm{KH}$, Meyer $\mathrm{B}$, Moldaenke $\mathrm{C}$, Lüring $\mathrm{C}$, Meyerhöfer M, Hansen U-P, Dau H (2002) A fluorometric method for the differentiation of algal populations in vivo and in situ. Photosynth Res 72:39-53

Bilger W, Björkman O (1990) Role of the xanthophylls cycle in photoprotection elucidated by measurements of light-induced absorbance changes, fluorescence and photosynthesis in leaves of Hedera canariensis. Photosynth Res 25:173-185

Bilger W, Björkman O (1991) Temperature dependence of violaxanthin de-epoxidation and non-photochemical fluorescence quenching in intact leaves of Gossypium hirsutum L. and Malva parviflora L. Planta 184:226-234

Bilger W, Schreiber U (1986) Energy-dependent quenching of darklevel chlorophyll fluorescence in intact leaves. Photosynth Res 10:303-308

Bilger W, Veit M, Schreiber L, Schreiber U (1997) Measurement of leaf epidermal transmittance of UV radiation by chlorophyll fluorescence. Physiol Plant 101:754-763

Björkman O, Demmig B (1987) Photon yield of $\mathrm{O}_{2}$ evolution and chlorophyll fluorescence characteristics at $77 \mathrm{~K}$ among vascular plants of diverse origins. Planta 170:489-504

Boisvert S, Joly D, Carpentier R (2006) Quantitative analysis of the experimental O-J-I-P chlorophyll fluorescence induction kinetics: apparent activation energy and origin of each step. FEBS J 273:4770-4777

Bonente G, Passarini F, Cazzaniga S, Mancone C, Buia MC, Tripodi M, Bassi R, Caffarri S (2008) The occurrence of the psbS gene product in Chlamydomonas reinhardtii and in other photosynthetic organisms and its correlation with energy quenching. Photochem Photobiol 84:1359-1370

Bonora A, Pancaldi S, Gualandri R, Fasulo MP (2000) Carotenoid and ultrastructure variations in plastids of Arum italicum Miller fruit during maturation and ripening. J Exp Bot 51:873-884

Bota J, Medrano H, Flexas J (2004) Is photosynthesis limited by decreased Rubisco activity and RuBP content under progressive water stress? New Phytol 162:671-681

Bradbury M, Baker NR (1981) Analysis of the slow phases of the in vivo chlorophyll fluorescence induction curve. Changes in the redox state of photosystem II electron acceptors and fluorescence emission from photosystems I and II. Biochim Biophys Acta 635:542-551

Brestic M, Zivcak M (2013) PSII fluorescence techniques for measurement of drought and high temperature stress signal in crop plants: protocols and applications. In: Rout GR, Das AB (eds) Molecular stress physiology of plants. Springer, Dordrecht, pp 87-131 
Brugnoli E, Björkman O (1992) Chloroplast movements in leaves: influence on chlorophyll fluorescence and measurements of light-induced absorbance changes related to $\Delta \mathrm{pH}$ and zeaxanthin formation. Photosynth Res 32:23-35

Buchanan BB (1984) The ferredoxin/thioredoxin system: a key element in the regulatory function of light in photosynthesis. BioScience 34:378-383

Bueno M, Fillat MF, Strasser RJ, Maldonado-Rodriguez R, Marina N, Smienk H, Gómez-Moreno C, Barja F (2004) Effects of lindane on the photosynthetic apparatus of the cyanobacterium Anabaena. Environ Sci Pollut Res 11:98-106

Bukhov NG, Govindachary S, Egorova EA, Joly D, Carpentier R (2003) $N, N, N^{\prime}, N^{\prime}$-tetramethyl-p-phenylenediamine initiates the appearance of a well resolved I peak in the kinetics of the fluorescence rise in isolated thylakoids. Biochim Biophys Acta 1607:91-96

Burling K, Hunsche M, Noga G (2011) Use of blue-green and chlorophyll fluorescence measurements for differentiation between nitrogen deficiency and pathogen infection in winter wheat. J Plant Physiol 168:1641-1648

Burrows PA, Sazonov LA, Svab Z, Maliga P, Nixon P (1998) Identification of a functional respiratory complex in chloroplasts through analysis of tobacco mutants containing disrupted plastid ndh genes. EMBO J 17:868-876

Buschmann C (1999) Photochemical and non-photochemical quenching coefficients of the chlorophyll fluorescence: comparison of variation and limits. Photosynthetica 37:217-224

Buschmann C (2007) Variability and application of the chlorophyll fluorescence emission ratio red/far-red of leaves. Photosynth Res 92:261-271

Buschmann C, Langsdorf G, Lichtenthaler HK (2001) Imaging of the blue, green, and red fluorescence emissionof plants: an overview. Photosynthetica 38:483-491

Bussotti F, Pollastrini M, Cascio C, Desotgiu R, Gerosa G, Marzuoli R, Nali C, Lorenzini G, Pellegrini E, Carucci MG, Salvatori E, Fusaro L, Piccotto M, Malaspina P, Manfredi A, Roccotello E, Toscano S, Gottardini E, Cristofori A, Fini A, Weber D, Baldassarre V, Barbanti L, Monti A, Strasser RJ (2011a) Conclusive remarks. Reliability and comparability of chlorophyll fluorescence data from several field teams. Environ Exp Bot 73:116-119

Bussotti F, Desotgiu R, Cascio C, Pollastrini M, Gravano E, Gerosa G, Marzuoli R, Nali C, Lorenzini G, Salvatori E, Manes F, Schaub M, Strasser RJ (2011b) Ozone stress in woody plants assessed with chlorophyll a fluorescence: a critical reassessment of existing data. Environ Exp Bot 73:19-30

Butler WL (1972) On the primary nature of fluorescence yield changes associated with photosynthesis. Proc Natl Acad Sci USA 69:3420-3422

Byrdin M, Rimke I, Schlodder E, Stehlik D, Roelofs TA (2000) Decay kinetics and quantum yields of fluorescence in photosystem I from Synechococcus elongatus with P700 in the reduced and oxidized state: are the kinetics of excited state decay traplimited or transfer-limited? Biophys J 79:992-1007

Caffarri S, Passarini F, Bassi R, Croce R (2007) A specific binding site for neoxanthin in the monomeric antenna proteins CP26 and CP29 of photosystem II. FEBS Lett 581:4704-4710

Caffarri S, Broess K, Croce R, van Amerongen H (2011) Excitation energy transfer and trapping in higher plant photosystem II complexes with different antenna sizes. Biophys J 100:2094-2103

Čajánek M, Štroch M, Lachetová I, Kalina J, Spunda V (1998) Characterization of the photosystem II inactivation of heatstressed barley leaves as monitored by the various parameters of chlorophyll a fluorescence and delayed fluorescence. J Photochem Photobiol B 47:39-45
Carillo N, Arana JL, Vallejos RH (1981) Light modulation of chloroplast membrane-bound ferredoxin-NADP ${ }^{+}$oxidoreductase. J Biol Chem 256:1058-1059

Caron L, Berkaloff C, Duval J-C, Jupin H (1987) Chlorophyll fluorescence transients from the diatom Phaeodactylum tricornutum: relative rates of cyclic phosphorylation and chlororespiration. Photosynth Res 11:131-139

Cassol D, de Silva FSP, Falqueto AR, Bacarin MA (2008) An evaluation of non-destructive methods to estimate total chlorophyll content. Photosynthetica 46:634-636

Cazzaniga S, dall'Osto L, Kong S-G, Wada M, Bassi R (2013) Interaction between avoidance of photon absorption, excess energy dissipation and zeaxanthin synthesis against photooxydative stress in Arabidopsis. Plant J 76:568-579

Ceppi MG (2010) Paramètres photosynthétiques affectant le transport d'électrons à travers le pool de plastoquinone: la densité des photosystèmes I, le contenu de chlorophylle et l'activité d'une plastoquinol-oxydase. PhD Thesis No 4175, University of Geneva, Geneva. Available at http://archive-ouverte.unige.ch/ unige p 5387

Ceppi MG, Oukarroum A, Çiçek N, Strasser RJ, Schansker G (2012) The IP amplitude of the fluorescence rise OJIP is sensitive to changes in the photosystem I content of leaves: a study on plants exposed to magnesium and sulfate deficiencies, drought stress and salt stress. Physiol Plant 144:277-288

Chaerle L, Hulsen K, Hermans C, Strasser RJ, Valcke R, Höfte M, van der Straeten D (2003) Robotized time-lapse imaging to assess in-plant uptake of phenylurea herbicides and their microbial degradation. Physiol Plant 118:613-619

Chow WS, Anderson JM, Melis A (1990a) The photosystem stoichiometry in thylakoids of some Australian shade-adapted plant species. Aust J Plant Physiol 17:665-674

Chow WS, Melis A, Anderson JM (1990b) Adjustments of photosystem stoichiometry in chloroplasts improve the quantum efficiency of photosynthesis. Proc Natl Acad Sci USA 87:7502-7506

Chylla RA, Garab G, Whitmarsh J (1987) Evidence for slow turnover in a fraction of photosystem II complexes in thylakoid membranes. Biochim Biophys Acta 894:562-571

Clark AJ, Landolt W, Bucher JB, Strasser RJ (2000) Beech (Fagus sylvatica L.) response to ozone exposure assessed with a chlorophyll $a$ fluorescence performance index. Environ Pollut 109:501-507

Croce R, Muller MG, Bassi R, Holzwarth AR (2001) Carotenoid-tochlorophyll energy transfer in recombinant major light-harvesting complex (LHCII) of higher plants. I. Femtosecond transient absorption measurements. Biophys J 80:901-915

Crofts AR, Wraight CA (1983) The electrochemical domain of photosynthesis. Biochim Biophys Acta 726:149-185

Cruz S, Goss R, Wilhelm C, Leegood R, Horton P, Jakob T (2010) Impact of chlororespiration on non-photochemical quenching of chlorophyll fluorescence and on the regulation of the diadinoxanthin cycle in the diatom Thalassiosira pseudonana. J Exp Bot 62:509-519

Cser K, Vass I (2007) Radiative and non-radiative charge recombination pathways in photosystem II studied by thermoluminescence and chlorophyll fluorescence in the cyanobacterium Synechocystis 6803. Biochim Biophys Acta 1767:233243

Daley PF, Raschke K, Ball JT, Berry JA (1989) Topography of photosynthetic activity of leaves obtained from video images of chlorophyll fluorescence. Plant Physiol 90:1233-1238

Dannehl H, Wietoska H, Heckmann H, Godde D (1996) Changes in D1-protein turnover and recovery of photosystem II activity precede accumulation of chlorophyll in plants after release from mineral stress. Planta 199:34-42 
de Weerd FL, Dekker JP, van Grondelle R (2003a) Dynamics of betacarotene-to-chlorophyll singlet energy transfer in the core of photosystem II. J Phys Chem B 107:6214-6220

de Weerd FL, Kennis JTM, Dekker JP, van Grondelle R (2003b) Beta-carotene to chlorophyll singlet energy transfer in the photosystem I core of Synechococcus elongatus proceeds via the beta-carotene S2 and S1 states. J Phys Chem B 107:5995-6002

Delosme R (1967) Étude de l'induction de fluorescence des algues vertes et des chloroplastes au début d'une illumination intense. Biochim Biophys Acta 143:108-128

Delosme R (1971) Variations du rendement de fluorescence de la chlorophylle in vivo sous l'action d'éclairs de forte intensité. Compt Rend Acad Sci Paris Sér D272:2828-2831

Delosme R (1972) New results about chlorophyll fluorescence in vivo. In: Forti G, Avron M, Melandri A (eds) Proceedings of the 2nd international congress on photosynthesis research, vol 1. Junk, The Hague, pp 187-195

Delrieu MJ (1998) Regulation of thermal dissipation of absorbed excitation energy and violaxanthin deepoxidation in the thylakoids of Lactuca sativa: photoprotective mechanism of a population of photosystem II centers. Biochim Biophys Acta 1363:157-173

Demmig B, Winter K (1988) Characterisation of three components of non-photochemical fluorescence quenching and their response to photoinhibition. Aust J Plant Physiol 15:163-177

Demmig-Adams B, Ebbert V, Zarter CR, Adams WW (2006) Characteristics and species-dependent employment of flexible versus sustained thermal dissipation and photoinhibition. In: Demmig-Adams B, Adams WW, Mattoo AK (eds) Photoprotection, photoinhibition, gene regulation, and environment, advances in photosynthesis and respiration, vol 21. Springer, Dordrecht, pp 39-48

Demmig-Adams B, Cohu CM, Muller O, Adams WW (2012) Modulation of photosynthetic energy conversion efficiency in nature: from seconds to seasons. Photosynth Res 113:75-88

Desotgiu R, Cascio C, Pollastrini M, Gerosa G, Marzuoli R, Bussotti F (2012) Short and long term photosynthetic adjustments in sun and shade leaves of Fagus sylvatica L., investigated with the fluorescence transient (FT) analysis. Plant Biosyst 146(Supp. 1):206-216

Dietzel L, Bräutigam K, Pfannschmidt T (2008) Photosynthetic acclimation: state transitions and adjustment of photosynthetic stoichiometry-functional relationships between short-term and long-term light quality acclimation in plants. FEBS J 275:1080-1088

Dinç E, Ceppi MG, Tóth SZ, Bottka S, Schansker G (2012) The chl $a$ fluorescence intensity is remarkably insensitive to changes in the chlorophyll content of the leaf as long as the chl $a / b$ ratio remains unaffected. Biochim Biophys Acta 1817:770-779

Diner B (1977) Dependence of the deactivation reactions of photosystem II on the redox state of plastoquinone pool A varied under anaerobic conditions: equilibria on the acceptor side of photosystem II. Biochim Biophys Acta 460:247-258

Drop B, Sathish Yadav KN, Boekema EJ, Croce R (2014) Consequences of state transitions on the structural and functional organization of photosystem I in the green alga Chlamydomonas reinhardtii. Plant J 78:181-191

Ducruet JM (1999) Relation between the heat-induced increase of $F_{0}$ fluorescence and a shift in the electronic equilibrium at the acceptor side of photosystem 2. Photosynthetica 37:335-338

Ducruet JM, Vass I (2009) Thermoluminescence: experimental. Photosynth Res 101:195-204

Duysens LNM, Sweers HE (1963) Mechanisms of two photochemical reactions in algae as studied by means of fluorescence. In: Studies on microalgae and photosynthetic bacteria, special issue of plant and cell physiology. Japanese Society of Plant Physiologists, University of Tokyo Press, Tokyo, pp 353-372

Earl HJ, Ennahli S (2004) Estimating photosynthetic electron transport via chlorophyll fluorometry without photosystem II light saturation. Photosynth Res 82:177-186

Edhofer I, Mühlbauer SK, Eichacker LA (1998) Light regulates the rate of translation elongation of chloroplast reaction center protein D1. Eur J Biochem 257:78-84

Elsheery NI, Wilske B, Zhang J-L, Cao K-F (2007) Seasonal variations in gas exchange and chlorophyll fluorescence in the leaves of five mango cultivars in southern Yunnan, China. J Hort Sci Biotechnol 82:855-862

Elzinga CL, Salzer DW, Willoughby JW, Gibbs JP (2001) Monitoring plant and animal populations. Blackwell Science, Malden, p 360

Emerson R, Lewis CM (1943) The dependence of the quantum yield of Chlorella photosynthesis on wavelength of light. Am J Bot 30:165-178

Etienne A-L, Ducruet J-M, Ajlani G, Vernotte C (1990) Comparative studies on electron transfer in photosystem II of herbicideresistant mutants from different organisms. Biochim Biophys Acta 1015:435-440

Evans JR (1986) A quantitative analysis of light distribution between the two photosystems, considering variation in both the relative amounts of the chlorophyll-protein complexes and the spectral quality of light. Photobiochem Photobiophys 10:135-147

Evans JR (1999) Leaf anatomy enables more equal access to light and $\mathrm{CO}_{2}$ between chloroplasts. New Phytol 143:93-1904

Evans JR, Loreto $\mathrm{F}$ (2000) Acquisition and diffusion of $\mathrm{CO}_{2}$ in higher plant leaves. In: Leegood RC, Sharkey TD, von Caemmerer S (eds) Photosynthesis: physiology and metabolism. Kluwer, Dordrecht, pp 321-351

Falkowski PG, Kolber Z (1990) Phytoplankton photosynthesis in the Atlantic Ocean as measured from a submersible pump and probe fluorometer in situ. In: Baltscheffsky M (ed) Current research in photosynthesis, vol V. Kluwer, Dordrecht, pp 923-926

Feild TS, Nedbal L, Ort DR (1998) Nonphotochemical reduction of the plastoquinone pool in sunflower leaves originates from chlororespiration. Plant Physiol 116:1209-1218

Ferroni L, Baldisserotto C, Pantaleoni L, Billi P, Fasulo MP, Pancaldi S (2007) High salinity alters chloroplast morpho-physiology in freshwater Kirchneriella species (Selenastraceae) from Ethiopian Lake Awasa. Am J Bot 94:1972-1983

Ferroni L, Baldisserotto C, Pantaleoni L, Fasulo MP, Fagioli P, Pancaldi S (2009) Degreening of the unicellular alga Euglena gracilis: thylakoid composition, room temperature fluorescence spectra and chloroplast morphology. Plant Biol 11:631-641

Ferroni L, Baldisserotto C, Giovanardi M, Pantaleoni L, Morosinotto T, Pancaldi S (2011) Revised assignment of room-temperature chlorophyll fluorescence emission bands in single living cells of Chlamydomonas reinhardtii. $\mathrm{J}$ Bioenergy Biomembr 43:163-173

Ferroni L, Pantaleoni L, Baldisserotto C, Aro E-M, Pancaldi S (2013) Low photosynthetic activity is linked to changes in the organization of photosystem II in the fruit of Arum italicum. Plant Physiol Biochem 63:140-150

Fey V, Wagner R, Bräutigam K, Pfannschmidt T (2005) Photosynthetic redox control of nuclear gene expression. J Exp Bot 56:1491-1498

Flexas J, Escalona JM, Medrano H (1998) Down-regulation of photosynthesis by drought under field conditions in grapevine leaves. Aust J Plant Physiol 25:893-900

Flexas J, Ribas-Carbó M, Hanson DT, Bota J, Otto B, Cifre J, McDowell N, Medrano H, Kaldenhoff R (2006) Tobacco aquaporin NtAQP1 is involved in mesophyll conductance to $\mathrm{CO}_{2}$ in vivo. Plant $\mathrm{J} 48: 427-439$ 
Flexas J, Ribas-Carbó M, Diaz-Espejo A, Galmés J, Medrano H (2008) Mesophyll conductance to $\mathrm{CO}_{2}$ : current knowledge and future prospects. Plant Cell Environ 31:602-621

Fork DC, Satoh K (1986) The control by state transitions of the distribution of excitation energy in photosynthesis. Annu Rev Plant Physiol 37:335-361

Franck F, Juneau P, Popovich R (2002) Resolution of the photosystem I and photosystem II contributions to chlorophyll fluorescence of intact laves at room temperature. Biochim Biophys Acta 1556:239-246

Franck F, Dewez D, Popovic R (2005) Changes in the roomtemperature emission spectrum of chlorophyll during fast and slow phases of the Kautsky effect in intact leaves. Photochem Photobiol 81:431-436

Fukshansky L, Martinez von Remisowsky A (1992) A theoretical study of the light microenvironment in a leaf in relation to photosynthesis. Plant Sci 86:167-182

Galmés J, Abadía A, Medrano H, Flexas J (2007) Photosynthesis and photoprotection responses to water stress in the wild-extinct plant Lysimachia minoricensis. Environ Exp Bot 60:308-317

Gasanov R, Abilov ZK, Gazanchyan RM, Kurbonova UM, Khanna R, Govindjee (1979) Excitation energy transfer in photosystems I and II from grana and in photosystem I from stroma lamellae, and identification of emission bands with pigment protein complexes. Z Pflanzenphysiol 95:148-169

Geel C, Versluis W, Snel JFH (1997) Estimation of oxygen evolution by marine phytoplankton from measurement of the efficiency of photosystem II electron flow. Photosynth Res 51:61-70

Genty B, Meyer S (1995) Quantitative mapping of leaf photosynthesis using imaging. Aust J Plant Physiol 22:277-284

Genty B, Briantais J-M, Baker NR (1989) The relationship between the quantum yield of photosynthetic electron transport and quenching of chlorophyll fluorescence. Biochim Biophys Acta 990:87-92

Genty B, Wonders J, Baker NR (1990) Non-photochemical quenching of $F_{\mathrm{O}}$ in leaves is emission wavelength dependent. Consequences for quenching analysis and its interpretation. Photosynth Res 26:133-139

Gielen B, Vandermeiren K, Horemans N, D'Haese D, Serneels R, Valcke R (2006) Chlorophyll $a$ fluorescence imaging of ozonestressed Brassica napus L. plants differing in glucosinolate concentrations. Plant Biol 8:698-705

Gilmore AM (2004) Excess light stress: probing excitation dissipation mechanisms through global analysis of time- and wavelengthresolved chlorophyll $a$ fluorescence. In: Govindjee, Papageorgiou GC (eds) Chlorophyll $a$ fluorescence: a signature of photosynthesis. Springer, Dordrecht, pp 555-581

Gilmore AM, Shinkarev VP, Hazlett TL, Govindjee (1998) Quantitative analysis of the effects of intrathylakoid $\mathrm{pH}$ and xanthophyll cycle pigments on chlorophyll $a$ fluorescence lifetime distributions and intensity in thylakoids. Biochemistry 37:13582-13593

Gitelson AA, Buschmann C, Lichtenthaler HK (1999) The chlorophyll fluorescence ratio F735/F700 as an accurate measure of the chlorophyll content in plants. Remote Sens Environ 69:296-302

Goltsev V, Zaharieva I, Chernev P, Strasser RJ (2009) Delayed fluorescence in photosynthesis. Photosynth Res 101:217-232

Goltsev V, Zaharieva I, Chernev P, Koezmanova M, Kalaji HM, Yordanov I, Krasteva V, Alexandrov V, Stefanov D, Allakhverdiev SI, Strasser RJ (2012) Drought-induced modifications of photosynthetic electron transport in intact leaves: analysis and use of neural networks as a tool for a rapid non-invasive estimation. Biochim Biophys Acta 1817:1490-1498

Gorbe E, Calatayud A (2012) Applications of chlorophyll fluorescence imaging technique in horticultural research: a review. Sci Hortic 138:24-35
Gotoh E, Matsumoto M, Ogawa K, Kobayashi Y, Tsuyama M (2010) A qualitative analysis of cyclic electron flow around photosystem I from the post-illumination chlorophyll fluorescence transients in Arabidopsis: a new platform for the in vivo investigation of the chloroplast redox state. Photosynth Res 103:111-123

Gottardini E, Cristofori A, Cristofolini F, Nali C, Pellegrini E, Busotti F, Ferretti M (2014) Chlorophyll-related indicators are linked tot visible ozone symptoms: evidence from a field study on native Viburnum lantana L. plants in northern Italy. Ecol Indic 39:65-74

Govindjee (1995) Sixty-three years since Kautsky: chlorophyll $a$ fluorescence. Aust J Plant Physiol 22:131-160

Govindjee (2004) Chlorophyll $a$ fluorescence: a bit of basics and history. In: Papageorgiou GC, Govindjee (eds) Chl $a$ fluorescence: a signature of photosynthesis, advances in photosynthesis and respiration, vol 19. Springer, Dordrecht, pp 1-42

Gray GR, Savitch LV, Ivanov AG, Huner NPA (1996) Photosystem II excitation pressure and development of resistance to photoinhibition. Plant Physiol 110:61-71

Greer DH, Berry JA, Björkman O (1986) Photoinhibition of photosynthesis in intact bean leaves: role of light and temperature, and requirement for chloroplast-protein synthesis during recovery. Planta 168:253-260

Groot ML, Frese RN, de Weerd FL, Bromek K, Petterson Å, Peterman EJG, van Stokkum IHM, van Grondelle R, Dekker JP (1999) Spectroscopic properties of the CP43 core antenna protein of photosystem II. Biophys J 77:3328-3340

Guarini JM, Moritz C (2009) Modelling the dynamics of the electron transport rate measured by PAM fluorimetry during rapid light curve experiments. Photosynthetica 47:206-214

Guidi L, Degl'Innocenti E (2011) Imaging of chlorophyll a fluorescence: a tool to study abiotic stress in plants. In: Shanker A (ed) Abiotic stress in plants-mechanisms and adaptations. InTech, Available from: http://www.intechopen.com/articles/show/title/ imaging-of-chlorophyll-a-fluorescence-a-tool-to-study-abioticstress-in-plants

Guidi L, Degl'Innocenti E (2012) Chlorophyll $a$ fluorescence in abiotic stress. In: Venkateswarlu B, Shanker AK, Shanker C, Maheswari M (eds) Crop stress and its management: perspectives and strategies. Springer, Dordrecht, pp 359-398

Guidi L, Mori S, Degl'Innocenti E, Pecchia S (2007) Effects of ozone exposure or fungal pathogen on white lupin leaves as determined by imaging of chlorophyll $a$ fluorescence. Plant Physiol Biochem $45: 851-857$

Guissé B, Srivastava A, Strasser RJ (1995) The polyphasic rise of the chlorophyll $a$ fluorescence (O-K-J-I-P) in heat-stressed leaves. Archs Sci Genève 48:147-160

Hagen SF, Solhaug KA, Bengtsson GB, Borge GIA, Bilger W (2006) Chlorophyll fluorescence as a tool for non-destructive estimation of anthocyanins and total flavonoids in apples. Postharvest Biol Technol 41:156-163

Hakala M, Tuomine I, Keränen M, Tyystjärvi T, Tyystjärvi E (2005) Evidence for the role of the oxygen-evolving manganese complex in photoinhibition of photosystem II. Biochim Biophys Acta 1706:68-80

Harbinson J (2013) Improving the accuracy of chlorophyll fluorescence measurements. Plant Cell Environ 36:1751-1754

Hart SE, Schlarb-Ridley BG, Bendall DS, Howe CJ (2005) Terminal oxidases of cyanobacteria. Biochem Soc Trans 33:832-835

Havaux M (1989) Increased thermal deactivation of excited pigments in pea leaves subjected to photoinhibitory treatment. Plant Physiol 89:286-292

Hemelrijk PW, Kwa SLS, van Grondelle R, Dekker JP (1992) Spectroscopic properties of LHC-II, the main light harvesting chlorophyll $a / b$ protein complex from chloroplast membranes. Biochim Biophys Acta 1098:159-166 
Hideg E, Schreiber U (2007) Parallel assessment of ROS formation and photosynthesis in leaves by fluorescence imaging. Photosynth Res 92:103-108

Hogewoning SW, Harbinson J (2007) Insights on the development, kinetics, and variation of photoinhibition using chlorophyll fluorescence imaging of a chilled, variegated leaf. J Exp Bot $58: 453-463$

Hogewoning SW, Wientjes E, Douwstra P, Trouwborst G, van Ieperen W, Croce R, Harbinson J (2012) Photosynthetic quantum yield dynamics: from photosystems to leaves. Plant Cell 24:1921-1935

Holzwarth AR (1996) Data analysis of time-resolved measurements. In: Amesz J, Hoff AJ (eds) Biophysical techniques in photosynthesis. Kluwer, The Netherlands, pp 75-92

Holzwarth AR (2008) Ultrafast primary reactions in the photosystems of oxygen-evolving organisms. In: Braun M, Gilch P, Zinth W (eds) Ultrafast laser pulses in biology and medicine. Springer, Berlin, pp 141-164

Holzwarth AR, Lenk D, Jahns P (2013) On the analysis of nonphotochemical chlorophyll fluorescence quenching curves: I. Theorethical considerations. Biochim Biophys Acta 1827:786-792

Horton P, Hague A (1988) Studies on the induction of chlorophyll fluorescence in isolated barley protoplasts: IV. Resolution of nonphotochemical quenching. Biochim Biophys Acta 932:107-115

Horton P, Ruban AV, Walters RG (1996) Regulation of light harvesting in green plants. Annu Rev Plant Physiol Plant Mol Biol 47:655-684

Hsu B-D, Leu K-L (2003) A possible origin of the middle phase of polyphasic chloropyll fluorescence transient. Funct Plant Biol 30:571-576

Hunsche M, Burling K, Noga G (2011) Spectral and time-resolved fluorescence signature of four weed species as affected by selected herbicides. Pest Biochem Phys 101:39-47

Inada K (1976) Action spectra for photosynthesis in higher plants. Plant Cell Physiol 17:355-365

Ioannidis N, Schansker G, Barynin VV, Petrouleas V (2000) Interaction of nitric oxide with the oxygen evolving complex of photosystem II and manganese catalase: a comparative study. J Bioinorg Chem 5:354-363

Iwai M, Takahashi Y, Minagawa Y (2008) Molecular remodeling of photosystem II during state transitions in Chlamydomonas reinhardtii. Plant Cell 20:2177-2189

Jakob T, Goss R, Wilhelm C (1999) Activation of diadinoxanthin deepoxidase due to a chlororespiratory proton gradient in the dark in the diatom Phaeodactylum tricornutum. Plant Biol 1:76-82

Johnson MP, Goral TK, Duffy CD, Brain AP, Mullineaux CW, Ruban AV (2011) Photoprotective energy dissipation involves the reorganization of photosystem II light-harvesting complexes in the grana membranes of spinach chloroplasts. Plant Cell 23:1468-1479

Joliot PA, Finazzi G (2010) Proton equilibration in the chloroplast modulates multiphasic kinetics of nonphotochemical quenching of fluorescence in plants. Proc Natl Acad Sci USA 107:12728-12733

Joly D, Carpentier R (2009) Sigmoidal reduction kinetics of the photosystem II acceptor side in intact photosynthetic materials during fluorescence induction. Photochem Photobiol Sci 8:167-173

Kalaji MH (2011) The impact of abiotic stress factors on the fluorescence of chlorophyll in plants of selected varieties of barley (Hordeum vulgare L.). Warsaw University of Life Sciences SSGW, Warsaw, (in Polish)

Kalaji MH, Guo P (2008) Chlorophyll fluorescence: a useful tool in barley plant breeding programs. In: Sanchez A, Guttierez SJ (eds) Photochemistry research in progress. Nova, New York, pp 439-463

Kalaji MH, Loboda T (2010) Chlorophyll fluorescence to study of the physiological status of plants. Warsaw Agricultural University, Warsaw, p 116

Kalaji MH, Bosa K, Koscielniak J, Hossain Z (2011a) Chlorophyll a fluorescence - a useful tool for the early detection of temperature stress in spring barley (Hordeum vulgare L.). OMICS J Integr Biol 15:925-934

Kalaji MH, Govindjee, Bosa K, Kościelniak J, Żuk-Gołaszewska K (2011b) Effects of salt stress on photosystem II efficiency and $\mathrm{CO}_{2}$ assimilation of two Syrian barley landraces. Environ Exp Bot 73:64-72

Kalaji MH, Carpentier R, Allakhverdiev SI, Bosa K (2012a) Fluorescence parameters as an early indicator of light stress in barley. J Photochem Photobiol B 112:1-6

Kalaji MH, Goltsev V, Bosa K, Allakhverdiev SI, Strasser RJ, Govindjee (2012b) Experimental in vivo measurements of light emission in plants: a perspective dedicated to David Walker. Photosynth Res 114:69-96

Kasahara M, Kagawa T, Oikawa K, Suetsuga N, Miyao M, Wada M (2002) Chloroplast avoidance movement reduces photodamage in plants. Nature 420:829-832

Kato MC, Hikosaka K, Hirose T (2002) Leaf discs floated on water are different from intact leaves in photosynthesis and photoinhibition. Photosynth Res 72:65-70

Kautsky H, Appel W, Amann H (1960) Chlorophyllfluoreszenz und Kohlensäure-assimilation: XIII. Die Fluoreszenzkurve und die Photochemie der Pflanze. Biochem Z 322:277-292

Kirova M, Ceppi G, Chernev P, Goltsev V, Strasser RJ (2009) Using artificial neural networks for plant taxonomic determination based on chlorophyll fluorescence induction curves. Biotechnol Biotechnol Equip 23:941-945

Kitajima M, Butler WL (1975) Quenching of chlorophyll fluorescence and primary photochemistry in chloroplasts by dibromothymoquinone. Biochim Biophys Acta 376:105-115

Kok B, Forbush B, McGloin M (1970) Cooperation of charges in photosynthetic $\mathrm{O}_{2}$ evolution. I. A linear four-step mechanism. Photochem Photobiol 11:467-475

Kolb CA, Kopecky J, Riederer M, Pfündel EE (2003) UV screening by phenolics in berries of grapevine (Vitis vinifera). Funct Plant Biol 30:1177-1186

Kolber ZS, Prášil O, Falkowski PG (1998) Measurements of variable chlorophyll fluorescence using fast repetition rate techniques: defining methodology and experimental protocols. Biochim Biophys Acta 1367:88-106

Krall JP, Edwards GE (1992) Relationship between photosystem II activity and $\mathrm{CO}_{2}$ fixation in leaves. Physiol Plant 86:180-187

Kramer DM, Johnson G, Kiirats O, Edwards GE (2004) New fluorescence parameters for the determination of $Q_{\mathrm{A}}$ redox state and excitation energy fluxes. Photosynth Res 79:209-218

Krause GH, Jahns P (2004) Non-photochemical energy dissipation determined by chlorophyll fluorescence quenching: characterization and function. In: GC Papageorgiou, Govindjee (eds) Chlorophyll a fluorescence: a signature of photosynthesis, advances in photosynthesis and respiration, vol 19. Springer, Berlin, pp 463-495

Krause GH, Briantais J-M, Vernotte C (1983) Characterization of chlorophyll fluorescence quenching in chloroplasts by fluorescence spectroscopy at $77 \mathrm{~K}$. I. $\Delta \mathrm{pH}$-dependent quenching. Biochim Biophys Acta 723:169-175

Krausz E, Hughes JL, Smith PJ, Pace RJ, Årsköld SP (2005) Assignment of the low-temperature fluorescence in oxygenevolving photosystem II. Photosynth Res 84:193-199

Kromkamp JC, Forster RM (2003) The use of variable fluorescence measurements in aquatic ecosystems: differences between 
multiple and single turnover measuring protocols and suggested terminology. Eur J Phycol 38:103-112

Kuroda H, Inagaki N, Satoh K (1992) The level of stromal ATP regulates translation of the D1 protein in isolated chloroplasts. Plant Cell Physiol 33:33-39

Kurreck J, Schödel R, Renger G (2000) Investigation of the plastoquinone pool size and fluorescence quenching in thylakoid membranes and photosystem II (PS II) membrane fragments. Photosynth Res 63:171-182

Laisk A, Loreto F (1996) Determining photosynthetic parameters from leaf $\mathrm{CO}_{2}$ exchange and chlorophyll fluorescence. Plant Physiol 110:903-912

Laisk A, Oja V (1998) Dynamics of leaf photosynthesis. CSIRO, Collingwood

Laisk A, Eichelmann H, Oja V, Rasulov B, Padu E, Bichele I, Pettai $\mathrm{H}$, Kull O (2005) Adjustment of leaf photosynthesis to shade in natural canopy: rate parameters. Plant Cell Environ 28:375-388

Lakowicz JR (2009) Principles of fluorescence spectroscopy, 3rd edn. Springer, Berlin

Landi M, Pardossi A, Remorini D, Guidi L (2013) Antioxidant and photosynthetic response of a purple-leaved and a green-leaved cultivar of sweet basil (Ocimum basilicum) to boron excess. Environ Exp Bot 85:64-75

Lavergne J (1982a) Two types of primary acceptor in chloroplast photosystem II. I. Different recombination properties. Photobiochem Photobiophys 3:257-271

Lavergne J (1982b) Mode of action of 3-(3,4-dichlorophenyl)-1,1dimethylurea. Evidence that the inhibitor competes with plastoquinone for binding to a common site on the acceptor side of photosystem II. Biochim Biophys Acta 682:345-353

Lavergne J, Leci E (1993) Properties of inactive photosystem II centers. Photosynth Res 35:323-343

Lazár D (2003) Chlorophyll $a$ fluorescence rise induced by high light illumination of dark-adapted plant tissue studied by means of a model of photosystem II and considering photosystem II heterogeneity. J Theor Biol 220:469-503

Lazár D, Schansker G (2009) Models of chlorophyll $a$ fluorescence transients. In: Laisk A, Nedbal L, Govindjee (eds) Photosynthesis in silico: understanding complexity from molecules to ecosystems, advances in photosynthesis and respiration, vol 29. Springer, Dordrecht, pp 85-123

Lazár D, Ilík P, Nauš J (1997) An appearance of K-peak in fluorescence induction depends on the acclimation of barley leaves to higher temperatures. J Lum 72-74:595-596

Lee W-J, Whitmarsh J (1989) Photosynthetic apparatus of pea thylakoid membranes. Plant Physiol 89:932-940

Lenk S, Chaerle L, Pfündel EE, Langsdorf G, Hagenbeek D, Lichtenthaler HK, van der Straeten D, Buschmann C (2007) Multispectral fluorescence and reflectance imaging at the leaf level and its possible application. J Exp Bot 58:807-814

Leong T-Y, Anderson JM (1984a) Adaptation of the thylakoid membranes of pea chloroplasts to light intensities. I. Study on the distribution of chlorophyll-protein complexes. Photosynth Res 5:105-115

Leong T-Y, Anderson JM (1984b) Adaptation of the thylakoid membranes of pea chloroplasts to light intensities. II. Regulation of electron transport capacities, electron carriers, coupling factor (CF1) activity and rates of photosynthesis. Photosynth Res 5:117-128

Lichtenthaler HK, Lang M, Sowinska M, Summ P, Heisel F, Miehe JA (1997) Uptake of the herbicide diuron as visualized by the fluorescence imaging technique. Bot Acta 110:158-163

Lichtenthaler HK, Buschmann C, Knapp M (2005) How to correctly determine the different chlorophyll fluorescence parameters and the chlorophyll fluorescence decrease ratio RFd of leaves with the PAM fluorometer. Photosynthetica 43:379-393
Logan BA, Demmig-Adams B, Rosenstiel TN, Adams WW (1999) Effect of nitrogen limitation on foliar antioxidants in relationship to other metabolic characteristics. Planta 209:213-220

Logan BA, Adams WW, Demmig-Adams B (2007) Avoiding common pitfalls of chlorophyll fluorescence analysis under field conditions. Funct Plant Biol 34:853-859

Lokstein H, Härtel H, Hoffmann P (1993) Comparison of chlorophyll fluorescence quenching in leaves of wild-type with a chlorophyll- $b$-less mutant of barley (Hordeum vulgare L.). J Photochem Photobiol B 19:217-225

Long SP, Humphries S, Falkowski PG (1994) Photoinhibition of photosynthesis in nature. Annu Rev Plant Physiol Plant Mol Biol 45:633-662

Loreto F, Harley PC, Di Marco G, Sharkey TD (1992) Estimation of mesophyll conductance to $\mathrm{CO}_{2}$ flux by three different methods. Plant Physiol 98:1437-1443

Loriaux S, Avenson T, Welles J, McDermitt D, Eckles R, Riensche B, Genty B (2013) Closing in on maximum yield of chlorophyll fluorescence using a single multiphase flash of sub-saturating intensity. Plant Cell Environ 36:1755-1770

Luyssaert S, Raitio H, Vervaeke P, Mertens J, Lust N (2002) Sampling procedure for the foliar analysis of deciduous trees. J Env Monitor 4:858-864

Malkin S (1966) Fluorescence induction studies in isolated chloroplasts; II. Kinetic analysis of the fluorescence intensity dependence on time. Biochim Biophys Acta 126:433-442

Mano J, Miyake C, Schreiber U, Asada K (1995) Photoactivation of the electron flow from NADPH to plastoquinone in spinach chloroplasts. Plant Cell Physiol 36:1589-1598

Markgraf T, Berry J (1990) Measurement of photochemical and nonphotochemical quenching: correction for turnover of PS2 during steadystate photosynthesis. In: Baltscheffsky M (ed) Current Research in Photosynthesis, vol IV. Kluwer, Dordrecht, pp 279-282

Marschall M, Proctor MCF (2004) Are bryophytes shade plants? Photosynthetic light responses and proportions of chlorophyll $a$, chlorophyll $b$ and total carotenoids. Ann Bot 94:593-603

Mauzerall D (1972) Light-induced changes in Chlorella, and the primary photoreaction for the production of oxygen. Proc Natl Acad Sci USA 69:1358-1362

Maxwell K, Johnson GN (2000) Chlorophyll fluorescence-a practical guide. J Exp Bot 51:659-668

McCree KJ (1972) The action spectrum, absorptance and quantum yield of photosynthesis in crop plants. Agric Meterol 9:191-216

Melis A (1991) Dynamics of photosynthetic membrane composition and function. Biochim Biophys Acta 1058:87-106

Meyer S, Genty B (1998) Mapping intercellular $\mathrm{CO}_{2}$ mole fraction $\left(\mathrm{C}_{\mathrm{i}}\right)$ in Rosa rubiginosa leaves fed with abscisic acid by using chlorophyll fluorescence imaging. Plant Physiol 116:947-957

Miloslavina Y, de Bianchi S, Dall'Osto L, Bassi R, Holzwarth AR (2011) Quenching in Arabidopsis thaliana mutants lacking monomeric antenna proteins of Photosystem II. J Biol Chem 286:36830-36840

Misra AN, Dilnawaz F, Misra M, Biswal AK (2001a) Thermoluminescence in chloroplasts as an indicator of alterations in photosystem 2 reaction centre by biotic and abiotic stress. Photosynthetica 39:1-9

Misra AN, Srivastava A, Strasser RJ (2001b) Utilisation of fast chlorophyll $a$ fluorescence technique in assessing the salt/ion sensitivity of mung bean and brassica seedlings. J Plant Physiol 158:1173-1181

Müller P, Li X-P, Niyogi KK (2001) Non-photochemical quenching. A response to excess light energy. Plant Physiol 125:1558-1566

Munday JCM, Jr, Govindjee (1969) Light-induced changes in the fluorescence yield of chlorophyll $a$ in vivo. III. The dip and the peak in the fluorescence transient of Chlorella pyrenoidosa. Biophys J 9:1-21 
Murata N, Nishimura M, Takamiya A (1966) Fluorescence of chlorophyll in photosynthetic systems; II. Induction of fluorescence in isolated spinach chloroplasts. Biochim Biophys Acta 120:23-33

Murchie EH, Lawson T (2013) Chlorophyll fluorescence analysis: a guide to good practice and understanding some new applications. J Exp Bot 64:3983-3998

Nakatani HY, Ke B, Dolan E, Arntzen CJ (1984) Identity of the photosystem II reaction center polypeptide. Biochim Biophys Acta 765:347-352

Nedbal L, Trtílek M, Kaftan D (1999) Flash fluorescence induction: a novel method to study regulation of photosystem II. J Photochem Photobiol B 48:154-157

Neubauer C, Schreiber U (1987) The polyphasic rise of chlorophyll fluorescence upon onset of the strong continuous illumination: I. Saturation characteristics and partial control by the photosystem II acceptor side. Z Naturforsch 42c:1246-1254

Nikiforou C, Manetas Y (2011) Inherent nitrogen deficiency in Pistacia lentiscus preferentially affects photosystem I: a seasonal field study. Funct Plant Biol 38:848-855

Nilkens M, Kress E, Lambrev P, Miloslavina Y, Müller M, Holzwarth AR, Jahns P (2010) Identification of a slowly inducible zeaxanthin-dependent component of non-photochemical quenching of chlorophyll fluorescence generated under steady-state conditions in Arabidopsis. Biochim Biophys Acta 1797:466-475

Nixon PJ, Rögner M, Diner BA (1991) Expression of a higher plant psbA gene in Synechocystis 6803 yields a functional hybrid photosystem II reaction center complex. Plant Cell 3:383-395

Nixon PJ, Michoux F, Yu J, Boehm M, Komenda J (2010) Recent advances in understanding the assembly and repair of photosystem II. Ann Bot 106:1-16

Niyogi K, Grossman A, Björkman O (1997) Chlamydomonas xanthophyll cycle mutants identified by video imaging of chlorophyll fluorescence quenching. Plant Cell 9:1369-1380

Niyogi K, Grossman A, Björkman O (1998) Arabidopsis mutants define a central role for the xanthophyll cycle in the regulation of photosynthetic energy conversion. Plant Cell 10:1121-1134

Noctor G, Rees D, Young A, Horton P (1991) The relationship between zeaxanthin, energy-dependent quenching of chlorophyll fluorescence, and trans-thylakoid $\mathrm{pH}$ gradient in isolated chloroplasts. Biochim Biophys Acta 1057:320-330

Ohnishi N, Allakhverdiev SI, Takahashi S, Higashi S, Watanabe M, Nishiyama Y, Murata N (2005) Two-step mechanism of photodamage to photosystem II: step 1 occurs at the oxygenevolving complex and step 2 occurs at the photochemical reaction center. Biochemistry 44:8494-8499

Osmond CB, Grace SC (1995) Perspective on photoinhibition and photorespiration in the field: quintessential inefficiencies of the light and dark reactions of photosynthesis? J Exp Bot 46:1351-1362

Oukarroum A, El Madidi S, Strasser RJ (2006) Drought stress induced in barley cultivars (Hordeum vulgare L.) by polyethylene glycol, probed by germination, root length and chlorophyll $a$ fluorescence rise (OJIP). Archs Sci Genève 59:65-74

Oukarroum A, Schansker G, Strasser RJ (2009) Drought stress effects on photosystem I content and photosystem II thermotolerance analyzed using chl $a$ fluorescence kinetics barley varieties differing in their drought tolerance. Physiol Plant 137:188-199

Ounis A, Cerovic ZG, Briantais JM, Moya I (2001) Dual-excitation FLIDAR for the estimation of epidermal UV absorption in leaves and canopies. Rem Sens Environ 76:33-48

Oxborough K (2004) Imaging of chlorophyll $a$ fluorescence: theoretical and practical aspects of an emerging technique for the monitoring of photosynthetic performance. J Exp Bot 55:1195-1205

Oxborough K, Baker NR (1997) Resolving chlorophyll a fluorescence images of photosynthetic efficiency into photochemical and non- photochemical components-calculation of $\mathrm{qP}$ and $F_{\mathrm{V}}^{\prime} / F_{\mathrm{M}}{ }^{\prime}$ without measuring $F_{0}{ }^{\prime}$. Photosynth Res 54:135-142

Pancaldi S, Baldisserotto C, Ferroni L, Bonora A, Fasulo MP (2002) Room-temperature microspectrofluorimetry as a useful tool for studying the assembly of the PSII chlorophyll-protein complexes in single living cells of etiolated Euglena gracilis Klebs during the greening process. J Exp Bot 53:1753-1763

Pantaleoni L, Ferroni L, Baldisserotto C, Aro EM, Pancaldi S (2009) Photosystem II organisation in chloroplasts of Arum italicum leaf depends on tissue location. Planta 230:1019-1031

Papageorgiou GC, Govindjee (eds) (2004) Chl a Fluorescence: a signature of photosynthesis, advances in photosynthesis and respiration, vol 19. Springer, Dordrecht

Papageorgiou GC, Govindjee (2011) Photosystem II fluorescence: slow changes - scaling from the past. J Photochem Photobiol B 104:258-270

Papageorgiou GC, Tsimilli-Michael M, Stamatakis K (2007) The fast and slow kinetics of chlorophyll $a$ fluorescence induction in plants, algae and cyanobacteria: a viewpoint. Photosynth Res 94:275-290

Perreault F, Oukarroum A, Pirastru L, Sirois L, Matias WG, Popovic R (2010) Evaluation of copper oxide nanoparticles toxicity using chlorophyll $a$ fluorescence imaging in Lemna gibba. J Bot 9

Petrouleas V, Crofts AR (2005) The iron-quinone acceptor complex. In: Wydrzynski TJ, Satoh K (eds) Photosystem II: the lightdriven water:plastoquinone oxidoreductase, Advances in photosynthesis and photorespiration, vol 22. Springer, Dordrecht, pp 177-206

Pettai H, Oja V, Freiberg A, Laisk A (2005) Photosynthetic activity of far-red light in green plants. Biochim Biophys Acta 1708:311-321

Pfündel E (1998) Estimating the contribution of photosystem I to total leaf chlorophyll fluorescence. Photosynth Res 56:185-195

Pfündel EE, Ghozlen NB, Meyer S, Cerovic ZG (2007) Investigating UV screening in leaves by two different types of portable UV fluorimeters reveals in vivo screening by anthocyanins and carotenoids. Photosynth Res 93:205-221

Pollastrini M, Holland V, Brüggeman W, Koricheva J, Jussila I, Scherer-Lorenzen M, Berger S, Bussotti F (2014) Interaction and competition processes among tree species in young experimental mixed forests, assessed with chlorophyll fluorescence and leaf morphology. Plant Biology 16:323-331

Potvin C (1985) Effect of leaf detachment on chlorophyll fluorescence during chilling experiments. Plant Physiol 78:883-886

Quilliam RS, Swarbrick PJ, Scholes JD, Rolfe SA (2006) Imaging photosynthesis in wounded leaves of Arabidopsis thaliana. J Exp Bot 57:55-69

Ralph PJ, Gademann R (2005) Rapid light curves: a powerful tool to assess photosynthetic activity. Aqua Bot 82:222-237

Rappaport F, Béal D, Joliot A, Joliot P (2007) On the advantages of using green light to study fluorescence yield changes in leaves. Biochim Biophys Acta 1767:56-65

Raschke K (1970) Stomatal responses to pressure changes and interruptions in the water supply of detached leaves of Zea mays L. Plant Physiol 45:415-423

Richter M, Goss R, Wagner B, Holzwarth AR (1999) Characterization of the fast and slow reversible components of nonphotochemical quenching in isolated pea thylakoids by picosecond time-resolved chlorophyll fluorescence analysis. Biochemistry 38:12718-12726

Rijgersberg CP, Amesz J, Thielen APGM, Swager JA (1979) Fluorescence emission spectra of chloroplasts and subchloroplast preparations at low temperature. Biochim Biophys Acta 545:473-482

Robinson HH, Crofts AR (1983) Kinetics of the oxidation-reduction reactions of photosystem II quinone acceptor complex, and the pathway for deactivation. FEBS Lett 153:221-226 
Roháček K (2010) Method for resolution and quantification of components of the non-photochemical quenching $\left(\mathrm{q}_{\mathrm{N}}\right)$. Photosynth Res 105:101-113

Roháček K, Soukupová J, Barták M (2008) Chlorophyll fluorescence: a wonderful tool to study plant physiology and plant stress. Res Signpost 37(661):41-104

Rolland A, Rimet F, Jacquet S (2010) A 2-year survey of phytoplankton in the Marne Reservoir (France): a case study to validate the use of an in situ spectrofluorometer by comparison with algal taxanomy and chlorophyll a measurements. Knowl Manag Aquat Ecosyst 398:2

Romanowska-Duda B, Kalaji MH, Strasser RJ (2005) The use of PSII activity of Spirodela oligorrhiza plants as an indicator for water toxicity. In: van der Est A, Bruce D (eds) Photosynthesis: fundamental aspects to global perspectives. Allen, Lawrence, pp 585-587

Romanowska-Duda ZB, Grzesik M, Kalaji MH (2010) Physiological activity of energy plants fertilized with sewage sludge and usefulness of the Phytotoxkit test in practice. Environ Prot Eng 36:73-81

Rosenqvist E, van Kooten O (2003) Chlorophyll fluorescence: a general description and nomenclature. In: DeEll JR, Toivonen PMA (eds) Practical applications of chlorophyll fluorescence in plant biology. Kluwer, Dordrecht, pp 31-77

Ruban AV, Horton P (1999) The xanthophyll cycle modulates the kinetics of nonphotochemical energy dissipation in isolated light-harvesting complexes, intact chloroplasts, and leaves of spinach. Plant Physiol 119:531-542

Rumeau D, Peltier G, Cournac L (2007) Chlororespiration and cyclic electron flow around PSI during photosynthesis and plant stress response. Plant Cell Environ 30:1041-1051

Sakai T, Kagawa T, Kasahara M, Swartz SE, Christie JM, Briggs WL, Wada M, Okada K (2001) Arabidopsis nph1 and npl1: blue light receptors that mediate both phototropism and chloroplast relocation. Proc Natl Acad Sci USA 98:6969-6974

Samson G, Bruce D (1996) Origin of the low yield of chlorophyll a fluorescence induced by single turnover flash in spinach thylakoids. Biochim Biophys Acta 1276:147-153

Samson G, Prášil O, Yaakoubd B (1999) Photochemical and thermal phases of chlorophyll $a$ fluorescence. Photosynthetica $37: 163-182$

Satoh K (1981) Fluorescence induction and activity of ferredoxin$\mathrm{NADP}^{+}$reductase in Bryopsis chloroplasts. Biochim Biophys Acta 638:327-333

Schansker G, Strasser RJ (2005) Quantification of non- $Q_{\mathrm{B}}$-reducing centers in leaves using a far-red pre-illumination. Photosynth Res 84:145-151

Schansker G, van Rensen JJS (1999) Performance of active photosystem II centers in photoinhibited pea leaves. Photosynth Res 62:175-184

Schansker G, Tóth SZ, Strasser RJ (2005) Methylviologen and dibromothymoquinone treatments of pea leaves reveal the role of photosystem I in the Chl $a$ fluorescence rise OJIP. Biochim Biophys Acta 1706:250-261

Schansker G, Tóth SZ, Strasser RJ (2006) Dark-recovery of the Chl $a$ fluorescence transient (OJIP) after light adaptation: the qTcomponent of non-photochemical quenching is related to an activated photosystem I acceptor side. Biochim Biophys Acta 1757:787-797

Schansker G, Yuan Y, Strasser RJ (2008) Chl $a$ fluorescence and $820 \mathrm{~nm}$ transmission changes occurring during a dark-to-light transition in pine needles and pea leaves: a comparison. In: Allen JF, Osmond B, Golbeck JH, Gantt E (eds) Energy from the Sun. Springer, Dordrecht, pp 951-955

Schansker G, Tóth SZ, Kovács L, Holzwarth AR, Garab G (2011) Evidence for a fluorescence yield change driven by a light- induced conformational change within photosystem II during the fast chlorophyll $a$ fluorescence rise. Biochim Biophys Acta 1807:1032-1043

Schansker G, Tóth SZ, Holzwarth AR, Garab G (2014) Chlorophyll $a$ fluorescence: beyond the limits of the $Q_{\mathrm{A}}$-model. Photosynth Res 120:43-58

Scheibe R (1990) Light/dark modulation: regulation of chloroplast metabolism in a new light. Bot Acta 103:327-334

Schmetterer G, Pils D (2004) Cyanobacterial respiration. In: Zannoni D (ed) Respiration in Archaea and Bacteria, vol 2: diversity of prokaryotic systems. Springer, Dordrecht, pp 261-278

Scholes JD, Rolfe SA (2009) Chlorophyll fluorescence imaging as tool for understanding the impact of fungal diseases on plant performance: a phenomics perspective. Funct Plant Biol $36: 880-892$

Schreiber U (1986) Detection of rapid induction kinetics with a new type of high-frequency modulated chlorophyll fluorometer. Photosynth Res 9:261-272

Schreiber U (1998) Chlorophyll fluorescence: new instruments for special applications. In: Garab G (ed) Photosynthesis: mechanisms and effects, vol V. Kluwer, Dordrecht, pp 4253-4258

Schreiber U (2002) Assessment of maximal fluorescence yield: donor-side dependent quenching and $Q_{\mathrm{B}}$-quenching. In: van Kooten O, Snel JFH (eds) Plant spectro-fluorometry: applications and basic research. Rozenberg, Amsterdam, pp 23-47

Schreiber U, Neubauer C (1987) The polyphasic rise of chlorophyll fluorescence upon onset of strong continuous illumination: II. Partial control by the photosystem II donor side and possible ways of interpretation. Z Naturforsch 42:1255-1264

Schreiber U, Fink R, Vidaver W (1977) Fluorescence induction in whole leaves: differentiation between the two leaf sides and adaptation to different light regimes. Planta 133:121-129

Schreiber U, Schliwa U, Bilger W (1986) Continuous recording of photochemical and non-photochemical chlorophyll fluorescence quenching with a new type of modulation fluorometer. Photosynth Res 10:51-62

Schreiber U, Endo T, Mi H, Asada K (1995) Quenching analysis of chlorophyll fluorescence by the saturation pulse method: particular aspects relating to the study of eukaryotic algae and cyanobacteria. Plant Cell Physiol 36:873-882

Schreiber U, Klughammer C, Kolbowski J (2012) Assessment of wavelength-dependent parameters of photosynthetic electron transport with a new type of multi-color PAM chlorophyll fluorometer. Photosynth Res 113:127-144

Schweitzer RH, Brudvig GW (1997) Fluorescence quenching by chlorophyll cations in photosystem II. Biochemistry 36:11351-11359

Serôdio J, Vieira S, Cruz S, Coelho H (2006) Rapid light-response curves of chlorophyll fluorescence in microalgae: relationship to steady-state light curves and non-photochemical quenching in benthic diatom-dominated assemblages. Photosynth Res 90:29-43

Serôdio J, Ezequiel J, Barnett A, Mouget J-L, Méléder V, Laviale M, Lavaud J (2012) Efficiency of photoprotection in microphytobenthos: role of vertical migration and the xanthophyll cycle against photoinhibition. Aquat Microb Ecol 67:161-175

Serôdio J, Ezequiel J, Frommlet J, Laviale M, Lavaud J (2013) A method for the rapid generation of nonsequential light-response curves of chlorophyll fluorescence. Plant Physiol 163:1089-1102

Shinkarev VP (2005) Flash-induced oxygen evolution in photosynthesis: simple solution for the extended S-state model that includes misses, double-hits, inactivation and backward-transitions. Biophys J 88:412-421

Shinkarev VP, Govindjee (1993) Insight into the relationship of chlorophyll $a$ fluorescence yield to the concentration of its natural quenchers in oxygenic photosynthesis. Proc Natl Acad Sci USA 90:7466-7469 
Šiffel P, Braunová Z (1999) Release and aggregation of the lightharvesting complex in intact leaves subjected to strong $\mathrm{CO}_{2}$ deficit. Photosynth Res 61:217-226

Snel JFH, Vos JH, Gylstra R, Brock TCM (1998) Inhibition of photosystem II (PSII) electron transport as a convenient endpoint to assess stress of the herbicide linuron on freshwater plants. Aquat Ecol 32:113-123

Špunda V, Čajánek M, Ilík P, Kalina J, Nauš J (1997) Appearance of long-wavelength excitation form of chlorophyll a in PS I fluorescence during greening of barley leaves under continuous light. J Photochem Photobiol B 40:149-153

Srivastava A, Guissé B, Greppin H, Strasser RJ (1997) Regulation of antenna structure and electron transport in photosystem II of Pisum sativum under elevated temperature probed by the fast polyphasic chlorophyll a fluorescence transient: OKJIP. Biochim Biophys Acta 1320:95-106

Srivastava A, Jüttner F, Strasser RJ (1998) Action of the allelochemical, fischerellin A, on photosystem II. Biochim Biophys Acta 1364:326-336

Srivastava A, Strasser RJ, Govindjee (1999) Greening of peas: parallel measurements of $77 \mathrm{~K}$ emission spectra, OJIP chlorophyll a fluorescence transient, period four oscillation of the initial fluorescence level, delayed light emission, and P700. Photosynthetica 37:365-392

Steffen R, Christen G, Renger G (2001) Time-resolved monitoring of flash-induced changes of fluorescence quantum yield and decay of delayed light emission in oxygen-evolving photosynthetic organisms. Biochemistry 40:173-180

Steffen R, Eckert H-J, Kelly AA, Dörmann P, Renger G (2005) Investigations on the reaction pattern of photosystem II in leaves from Arabidopsis thaliana by time-resolved fluorometric analysis. Biochemistry 44:3123-3133

Stirbet A (2013) Excitonic connectivity between photosystem II units: what is it, and how to measure it? Photosynth Res 116:189-214

Stirbet A, Govindjee (2011) On the relation between the Kautsky effect (chlorophyll $a$ fluorescence induction) and photosystem II: basics and applications of the OJIP fluorescence transient. J Photochem Photobiol B 104:236-257

Stirbet A, Govindjee R (2012) Chlorophyll $a$ fluorescence induction: a personal perspective of the thermal phase, the J-I-P rise. Photosynth Res 113:15-61

Stitt M, Huber S, Kerr P (1987) Control of photosynthetic sucrose synthesis. In: Hatch MD, Boardman NK (eds) The biochemistry of plants, vol 10. Academic Press, London, pp 327-409

Strasser BJ (1996) Photosystem II structure and function studied by fast fluorescence transients. Université de Genève, Travail de diplôme

Strasser RJ, Butler WL (1976) Energy transfer in the photochemical apparatus of flashed bean leaves. Biochim Biophys Acta 449:412-419

Strasser RJ, Govindjee (1991) The $F_{\mathrm{O}}$ and the OJIP fluorescence rise in higher plants and algae. In: Argyroudi-Akoyunoglou JH (ed) Regulation of chloroplast biogenesis. Plenum, New York, pp 423-426

Strasser RJ, Stirbet A (2001) Estimation of the energetic connectivity of PS II centres in plants using the fluorescence rise O-J-I-P: fitting of experimental data to three different PS II models. Math Comput Simul 56:451-462

Strasser BJ, Strasser RJ (1995) Measuring fast fluorescence transients to address environmental questions: the JIP test. In: Mathis P (ed) Photosynthesis: from light to biosphere, vol V. Kluwer, Dordrecht, pp 977-980

Strasser RJ, Srivastava A, Govindjee (1995) Polyphasic chlorophyll $a$ fluorescence transient in plants and cyanobacteria. Photochem Photobiol 61:32-42
Strasser RJ, Tsimilli-Michael M, Srivastava A (2004) Analysis of the chlorophyll a fluorescence transient. In: Papageorgiou G, Govindjee (eds) Chlorophyll $a$ fluorescence: a signature of photosynthesis, advances in photosynthesis and respiration. Springer, Dordrecht, pp 321-362

Streusand VJ, Portis AR (1987) Rubisco activase mediates ATPdependent activation of ribulose bisphosphate carboxylase. Plant Physiol 85:152-154

Stumpp MT, Motohashi K, Hisabori T (1999) Chloroplast thioredoxin mutants without active-site cysteins facilitate the reduction of the regulatory disulphide bridge on the $\gamma$-subunit of chloroplast ATP synthase. Biochem J 341:157-163

Suggett DJ, Prášil O, Borowitzka MA (eds) (2011) Chlorophyll $a$ fluorescence in aquatic sciences: methods and applications. Springer, Dordrecht

Sun J, Nishio JN, Vogelmann TC (1998) Green light drives $\mathrm{CO}_{2}$ fixation deep within leaves. Plant Cell Physiol 39:1020-1026

Sušila P, Lazár D, Ilík P, Tomek P, Nauš J (2004) The gradient of exciting radiation within a sample affects relative heights of steps in the fast chlorophyll $a$ fluorescence rise. Photosynthetica 42:161-172

Susplugas S, Srivastava A, Strasser RJ (2000) Changes in the photosynthetic activities during several stages of vegetative growth of Spirodela polyrhiza: effect of chromate. J Plant Physiol 157:503-512

Terashima I, Saeki T (1985) Vertical gradient in photosynthetic properties of spinach chloroplasts dependent on intra-leaf light environment. Plant Cell Physiol 26:781-785

Terashima I, Sakaguchi S, Hara N (1986) Intra-leaf and intracellular gradients in chloroplast ultrastructure of dorsiventral leaves illuminated from the adaxial or abaxial side during their development. Plant Cell Physiol 27:1023-1031

Terashima I, Fujita T, Inoue T, Chow WS, Oguchi R (2009) Green light drives leaf photosynthesis more efficiently than red light in strong white light: Revisiting the enigmatic question of why leaves are green. Plant Cell Physiol 50:684-697

Tóth SZ, Schansker G, Strasser RJ (2005a) In intact leaves, the maximum fluorescence level $\left(F_{\mathrm{M}}\right)$ is independent of the redox state of the plastoquinone pool: a DCMU-inhibition study. Biochim Biophys Acta 1708:275-282

Tóth SZ, Schansker G, Kissimon J, Kovács L, Garab G, Strasser RJ (2005b) Biophysical studies of photosystem II-related recovery processes after a heat pulse in barley seedling (Hordeum vulgare L). J Plant Physiol 162:181-194

Tóth SZ, Schansker G, Strasser RJ (2007a) A non-invasive assay of the plastoquinone pool redox state based on the OJIP-transient. Photosynth Res 93:193-203

Tóth SZ, Schansker G, Garab G, Strasser RJ (2007b) Photosynthetic electron transport activity in heat-treated barley leaves: the role of internal alternative electron donors to photosystem II. Biochim Biophys Acta 1767:295-305

Trissl HW, Wilhelm C (1993) Why do thylakoid membranes from higher plants form grana stacks? Trends Biochem Sci 18:415-419

Tuba Z, Saxena DK, Srivastava K, Singh S, Sz Czebol, Kalaji MH (2010) Chlorophyll $a$ fluorescence measurements for validating the tolerant bryophytes for heavy metal $(\mathrm{Pb})$ biomapping. Curr Sci 98:1505-1508

Tyystjärvi E, Aro EM (1996) The rate constant of photoinhibition, measured in lincomycin-treated leaves, is directly proportional to light intensity. Proc Natl Acad Sci USA 93:2213-2218

Tyystjärvi E, Koski A, Keränen M, Nevalainen O (1999) The Kautsky curve is a built-in bar code. Biophys J 77:1159-1167

van der Weij-de Wit CD, Ihalainen JA, van Grondelle R, Dekker JP (2007) Excitation energy transfer in native and unstacked 
thylakoid membranes studied by low temperature and ultrafast fluorescence spectroscopy. Photosynth Res 93:173-182

van Dorssen RJ, Breton J, Plijter JJ, Satoh K, van Gorkom HJ, Amesz J (1987) Spectroscopic properties of the reaction center and of the $47 \mathrm{kDa}$ chlorophyll protein of photosystem II. Biochim Biophys Acta 893:267-274

van Heerden PDR, Swanepoel JW, Krüger GHJ (2007) Modulation of photosynthesis by drought in two desert scrub species exhibiting $\mathrm{C}_{3}$-mode $\mathrm{CO}_{2}$ assimilation. Environ Exp Bot 61:124-136

van Kooten O, Snel JF (1990) The use of chlorophyll fluorescence nomenclature in plant stress physiology. Photosynth Res 25:147-150

van Wijk KJ, Krause GH (1991) Oxygen dependence of photoinhibition at low temperature in intact protoplasts of Valerianella locusta L. Planta 186:135-142

Vass I, Govindjee (1996) Thermoluminescence from the photosynthetic apparatus. Photosynth Res 48:117-126

Vass I, Sass L, Spetea C, Bakou A, Ghanotakis DF, Petrouleas V (1996) UV-B-induced inhibition of photosystem II electron transport studied by EPR and chlorophyll fluorescence: impairment of donor and acceptor side components. Biochemistry 35:8964-8973

Velthuys BR (1981) Electron-dependent competition between plastoquinone and inhibitors for binding to photosystem II. FEBS Lett 126:277-281

Verhoeven A, Demmig-Adams B, Adams WW (1997) Enhanced employment of the xanthophyll cycle and thermal energy dissipation in spinach exposed to high light and $\mathrm{N}$ stress. Plant Physiol 113:817-824

Vermaas WFJ (2001) Photosynthesis and respiration in cyanobacteria. Encyclopedia of the life sciences. McMillan, London

Vernotte C, Etienne AL, Briantais J-M (1979) Quenching of the system II chlorophyll fluorescence by the plastoquinone pool. Biochim Biophys Acta 545:519-527

Vogelmann TC (1989) Penetration of light into plants. Photochem Photobiol 50:895-902

Vogelmann TC (1993) Plant tissue optics. Annu Rev Plant Physiol Plant Mol Biol 44:231-251

Vogelmann TC, Evans JR (2002) Profiles of light absorption and chlorophyll within spinach leaves from chlorophyll fluorescence. Plant Cell Environ 25:1313-1323

Vogelmann TC, Han T (2000) Measurement of gradients of absorbed light in spinach leaves from chlorophyll fluorescence profiles. Plant Cell Environ 23:1303-1311

Vogelmann TC, Martin G (1993) The functional significance of palisade tissue: penetration of directional versus diffuse light. Plant Cell Environ 16:65-72

Vogelmann TC, Bornman JF, Yates DJ (1996) Focusing of light by leaf epidermal cells. Physiol Plant 98:43-56

von Caemmerer S (2000) Biochemical models of photosynthesis. CSIRO, Collingwood

Vredenberg WJ (2000) A three-state model for energy trapping and chlorophyll fluorescence in photosystem II incorporating radical pair recombination. Biophys J 79:26-38

Vredenberg WJ (2008) Algorithm for analysis of OJDIP fluorescence induction curves in terms of photo- and electrochemical events in photosystems of plant cells: derivation and application. J Photochem Photobiol B 91:58-65

Vredenberg W, Kasalicky V, Durchan M, Prasil O (2006) The chlorophyll a fluorescence induction pattern in chloroplasts upon repetitive single turnover excitations: accumulation and function of QB-nonreducing centers. Biochim Biophys Acta 1757:173-181

Wada M (2013) Chloroplast movement. Plant Sci 210:177-182

Walters RG, Horton P (1991) Resolution of components of nonphotochemical quenching chlorophyll fluorescence quenching in barley leaves. Photosynth Res 27:121-133

Walters RG, Horton P (1993) Theoretical assessment of alternative mechanisms for non-photochemical quenching of PSII fluorescence in barley leaves. Photosynth Res 36:119-139

Walters RG, Horton P (1994) Acclimation of Arabidopsis thaliana to the light environment: changes in composition of the photosynthetic apparatus. Planta 195:248-256

Walters RG, Horton P (1995) Acclimation of Arabidopsis thaliana to the light environment: changes in photosynthetic function. Planta 197:306-312

Warren $\mathrm{C}$ (2006) Estimating the internal conductance to $\mathrm{CO}_{2}$ movement. Funct Plant Biol 33:431-442

Weis E (1985) Chlorophyll fluorescence at $77 \mathrm{~K}$ in intact leaves: characterization of a technique to eliminate artifacts related to self-absorption. Photosynth Res 6:73-86

Weng J-H, Chien C-T, Chen C-W, Lai X-M (2011) Effects of osmotic and high-light stresses on PSII efficiency of attached and detached leaves of three tree species adapted to different water regimes. Photosynthetica 49:555-563

White AJ, Critchley C (1999) Rapid light curves: a new fluorescence method to assess the state of the photosynthetic apparatus. Photosynth Res 59:63-72

Wientjes E, van Amerongen H, Croce R (2013) LHCII is an antenna of both photosystems after long-term acclimation. Biochim Biophys Acta 1827:420-426

Wingler A, Marès M, Pourtau N (2004) Spatial patterns and metabolic regulation of photosynthetic parameters during leaf senescence. New Phytol 161:781-789

Woo NS, Badger MR, Pogson BJ (2008) A rapid, non-invasive procedure for quantitative assessment of drought survival using chlorophyll fluorescence. Plant Methods 4:27

Yamasaki T, Yamakawa T, Yamane Y, Koike H, Satoh K, Katoh S (2002) Temperature acclimation of photosynthesis and related changes in photosystem II electron transport in winter wheat. Plant Physiol 128:1087-1097

Zankel K (1973) Rapid fluorescence changes observed in chloroplasts: their relationship to the $\mathrm{O}_{2}$ evolving system. Biochim Biophys Acta 325:138-148

Zhu X-G, Baker NR, Govindjee, de Sturler E, Ort DR, Long SP (2005) Chlorophyll $a$ fluorescence induction kinetics in leaves predicted from a model describing each discrete step of excitation energy and electron transfer associated with photosystem II. Planta 223:114-133

Zubek S, Turnau K, Tsimilli-Michael M, Strasser RJ (2009) Response of endangered plant species to inoculation with arbuscular mycorrhizal fungi and soil bacteria. Mycorrhiza 19:113-123 